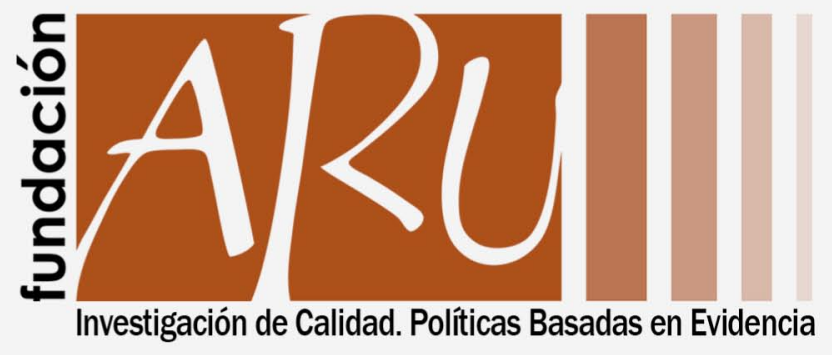

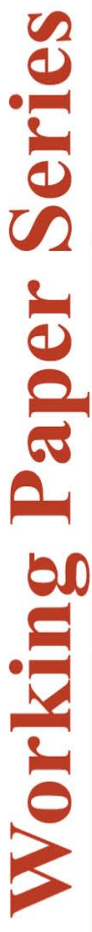

\title{
¿Libres de Analfabetismo? \\ Evaluando la Experiencia Boliviana con el Programa Nacional de Alfabetización "Yo Si Puedo"
}

\author{
Werner L. Hernani-Limarino \\ Christian Valencia \\ Paul Villarroel
}




\title{
¿Libres de Analfabetismo?
}

\section{Evaluando la Experiencia Boliviana con el Programa Nacional de Alfabetización "Yo Si Puedo"}

\author{
Free of Illiteracy?
}

Evaluating the Bolivian Experience with the National
Literacy Program "Yo Si Puedo"

\author{
Werner L. Hernani-Limarino* \\ Christian Valencia**, Paul Villarroel*** \\ Abril, 2013
}

\section{Resumen $^{1}$}

Este documento evalúa el éxito del Programa Nacional de Alfabetización (PNA) Yo Si Puedo. Por una parte, evaluamos si la implementación del PNA está asociada con un cambio en el nivel y la tendencia de la tasa de analfabetismo, y si dichos cambios fueron suficientes para reducir la incidencia del analfabetismo por debajo del $4 \%$ - nivel que per-mite declarar un país como libre de analfabetismo. Por otra parte, evaluamos el impacto del PNA en capacidades básicas de lectura y escritura. En el primer escenario, no encontramos evidencia que muestre que el PNA haya reducido ni los niveles globales de analfabetismo ni las tasas naturales de reducción del analfabetismo con excepción del caso de las mujeres en el área urbana. En el segundo escenario, las estimaciones del impacto del PNA son cuantitativamente pequeñas pero estadísticamente significativas en el indicador de lectura mecánica,

\footnotetext{
* Fundación ARU. Contacto: whl@aru.org.bo

*** Fundación ARU. Contacto: pvillarroel@aru.org.bo

${ }^{1}$ Los puntos de vista y opiniones contenidas en este documento no necesariamente reflejan la posición de la Fundación ARU ni de ninguna otra institución con la que los autores estén afiliados. Este documento fue elaborado para la Campaña Nacional por el Derecho a la Educación. Los autores agradecen a Ludwing Torres, Alvaro Chirino y la Red Boliviana de Microdatos y Encuestas su valiosa colaboración en el diseño e implementación de la Encuesta de Evaluación. Sin embargo, todos los errores u omisiones son de exclusiva responsabilidad de los autores.
} 
estadísticamente no diferentes de cero en los dos indicadores de lectura comprensiva, y cuantitativamente pequeñas pero estadísticamente significativas en los indicadores de comunicación escrita. Si bien nuestros resultados son inconsistentes con los pronunciamientos oficiales que declaran a Bolivia como territorio libre de analfabetismo, coinciden con otras evaluaciones que muestran la ineficacia e ineficiencia de los programas de alfabetización de adultos.

Palabras clave: Evaluación de impacto, programas de alfabetización, educación

\begin{abstract}
This paper evaluates the success of National Literacy Program (PNA) "Yo Si Puedo". First, we evaluate whether the implementation of PNA is associated with a change in the level and trend of illiteracy, and if such changes were sufficient to reduce the incidence of illiteracy below $4 \%$ - a level which permits declare a country free of illiteracy. Moreover, we evaluate the impact of PNA in basic reading and writing skills. In the first scenario, we found no evidence to show that PNA has reduced levels of illiteracy and natural rates of illiteracy reduction except for women in urban areas. In the second scenario, estimates show that PNA impact is quantitatively small but statistically significant on mechanical reading skills, statistically not different from zero on two reading comprehension outcomes, and quantitatively small but statistically significant on written communication skills. While our results are inconsistent with official statements that declare Bolivia as free of illiteracy, they are consistent with other evaluations showing the ineffectiveness and inefficiency of adult literacy programs.
\end{abstract}

Keywords: Impact evaluation, literacy programs, education

Clasificación/Classification (JEL): C21, I2, I3 


\section{Introducción}

El 20 de diciembre de 2009 autoridades de Gobierno declararon a Bolivia como "territorio libre de analfabetismo" marcando el éxito del Programa Nacional de Alfabetización (PNA) "Yo Si Puedo". El método diseñado por la educadora cubana Leonela Reyes habría conseguido 40 meses después del inicio de sus actividades la alfabetización de 824,101 jóvenes y adultos reduciendo la tasa de analfabetismo del país por debajo del $4 \% 2$. De esta forma, Bolivia se constituyó en el tercer país latinoamericano que se declaró libre de analfabetismo, ya que Cuba y Venezuela lo habían hecho en los años 1961 y 2005, respectivamente.

De comprobarse el éxito del PNA se demostraría que programas de alfabetización de adultos de escala nacional bien diseñados pueden ser sino eficientes al menos eficaces, y que el escepticismo de los organismos internacionales que redujeron el financiamiento de dichos pro-gramas al inicio de la década del 90 puede ser infundado3. Aún cuando existen argumentos a favor de los programas de alfabetización de adultos por su contribución al empoderamiento de individuos y comunidades, la mayoría de la literatura apunta no sólo a la ineficacia sino también a la ineficiencia de este tipo de programas. Por ejemplo, Romain y Armstrong (1987) encuentran que los programas de alfabetización de adultos han estado plagados de bajos niveles de matriculación, altos niveles de abandono y rápida perdida de las habilidades adquiridas. Abadzi (1994) encuentra que el porcentaje de aprobación en exámenes dentro de los programas de alfabetización fluctúa entre un mínimo de $8 \%$ y un máximo de $47 \%$. En una revisión de programas de alfabetización implementados durante las décadas del 60 y 70, Abadzi (2003) encuentra que pocos participantes fueron capaces de adquirir y retener habilidades básicas de lectura y comprensión y, escritura $\mathrm{y}$ comunicación, con estimaciones de niveles de eficiencia promedio de $12.5 \%$. Finalmente, Ortega y Rodriguez (2006) encuentran que, una vez que se controlan por la tendencia natural de los niveles de alfabetización, el programa de alfabetización de adultos "Misión Robinson" - la versión venezolana del método cubano "Yo Sí Puedo", no tuvo impactos ni cuantitativamente ni estadísticamente significativos ni en el nivel ni en la tendencia de la alfabetización en la mayoría de las especificaciones analizadas por los autores. Por otra parte, los documentos realizados en Bolivia encuentran diversos resultados para el Programa de Alfabetización, haciendo énfasis en posibles externalidades generadas por el pro-grama. Canfux y Liendo (2008) realizan una evaluación del impacto del Programa Nacional de Alfabetización midiendo el grado de desarrollo alcanzado en las personas, la familia, comunidad e instituciones involucradas en el proceso de alfabetización. La evaluación se realiza en base a los objetivos del programa y valora el impacto del programa en el desarrollo de las potencialidades individuales y sociales de los participantes a través de encuestas y entrevistas. Realizan un levantamiento de información primaria, recogiendo opiniones de los participantes en relación a los facilitadores, supervisores y el material

2 Comunica Bolivia(2009). Discurso del Presidente del Estado Plurinacional de Bolivia Evo Morales Ayma. Coliseo de la Coronilla, Ciudad de Cochabamba. 20 de diciembre del 2009.

3 Chowdury (1995) afirma que el escepticismo en los programas de alfabetización de adultos fue la causa fundamental de la fuerte reducción que efectuó el Banco Mundial al financiamiento de estos programas. 
provisto por el programa. Los resultados que encuentran estos autores hacen referencia a las potencialidades personales, a las relaciones interpersonales y a las potencialidades sociales. En estos tres aspectos, dicen los autores, se encuentran resultados positivos, y en un análisis para los departamentos de La Paz, Cochabamba y Santa Cruz los mismos no difieren.

Canfux, Liendo y Mora (2008) hacen una revisión del programa "Yo Si Puedo". Estudian la concepción teórica y metodológica del programa, para después pasar a la descripción de los participantes del PNA y hacer un análisis de la situación de analfabetismo en Bolivia. Finalmente, Mora y Canfux (2009) sistematizan la experiencia pedagógica y la gestión educativa que realizó el PNA entre 2006 y 2008. Se comentan aspectos técnicos del Programa haciendo especial énfasis en los facilitadores, supervisores y la implementación del programa. Resaltan que 823,256 personas fueron alfabetizadas, en su mayoría pertenecientes a sectores marginales y se encontraban excluidos del sistema educativo.

El objetivo de este documento es evaluar el éxito de la experiencia Boliviana con el PNA "Yo Si Puedo". El documento está organizado de la siguiente manera. La sección 2 describe la intervención y los productos del PNA. La sección 3 utiliza los datos de la serie armonizada de encuestas de hogares del periodo 1999-2009 para analizar no sólo las tendencias observadas en los niveles de analfabetismo auto-heteroevaluados, sino también para evaluar potenciales cambios en los niveles y las tendencias de analfabetismo atribuibles al PNA. Si bien resulta interesante utilizar datos oficiales con cobertura nacional para evaluar los resultados del PNA, existen serias limitaciones a las auto-heteroevaluaciones de la capacidad de las personas de leer y escribir. No sólo es posible que las personas sobreestimen sus propias capacidades - en cuyo caso las evaluaciones pueden sobre-estimar el impacto de los programas de alfabetización; sino también que las personas que participen del programa no sean completamente analfabetos sino analfabetos "funcionales", i.e. que los participantes del programa ya declaren que saben leer y escribir, en cuyo caso las evaluaciones de este tipo pueden sub-estimar el impacto del programa. Para superar esta dificultad, la sección 4 presenta una evaluación de las ganancias del PNA en el departamento de Chuquisaca a partir de evaluaciones directas de las capacidades de lectura y escritura. Como no fue posible realizar evaluaciones de los participantes antes del inicio de actividades del programa, nuestro método de cálculo del valor agregado del programa se ve obligado a asumir que, una vez que se controlan por factores de confusión observables, las capacidades promedio de lectura y escritura de la población no tratada son una buena aproximación a las capacidades promedio que se observarían en la población que participó del programa si no hubiera participado del programa. La sección 5 concluye. Los apéndices presentados al final del documento amplían la información sobre los métodos de estimación e inferencia utilizados.

\section{El Programa Nacional de Alfabetización}

\subsection{Arreglos Institucionales del PNA}

El PNA "Yo Sí Puedo" fue uno de los primeros programas educativos implementados durante la primera gestión de Gobierno de Evo Morales Ayma. El PNA fue establecido por Decreto Supremo N²8675 el 13 de abril de 2006 con el objetivo de "erradicar el analfabetismo" en el 
país4. Este Decreto Supremo también establece que el programa recibirá asistencia técnica de la República de Cuba en el diseño e implementación del dentro del país y dispone que todas las entidades públicas están obligadas a colaborar con las autoridades nacionales y cubanas para el cumplimiento de los objetivos del programa5. El programa fue ejecutado bajo tuición del Ministerio de Educación que estableció como unidad ejecutora a la Dirección de Alfabetización dependiente del Vice-Ministerio de Educación Escolarizada y Alfabetización.

\subsection{Financiamiento}

La Tabla 12 presenta información de los gastos, así como de las fuentes de financiamiento del PNA. Durante los cuatro años de duración del programa la mayor parte del gasto fue realizado por concepto de maquinaria y equipos, utilizados mayormente el primer año, este gasto puede ser atribuido a la instalación de paneles solares en las comunidades sin acceso a servicios de energía y a la adquisición del equipamiento necesario para el método audiovisual del PNA. Aproximadamente $2 / 3$ del financiamiento del programa provinieron de donaciones externas y el 1/3 restante del TGN. Las donaciones externas se concentraron durante el 2006 y fueron decreciendo los años siguientes. La mayor parte de estos recursos externos fueron donados por la República de Cuba.

\subsection{Tratamiento}

El PNA utilizó como tratamiento el método desarrollado por la pedagoga cubana Leonela Reyes denominado "Yo Sí Puedo". Canfux (2008) describe el método como uno de alfabetización masiva que utiliza fundamentalmente métodos audiovisuales para desarrollar las relaciones entre escuchar y ver (oído-ojo); escuchar y leer (oído-libro); y escuchar y escribir (oído-lápiz). En particular el método utiliza 17 videos en 65 clases para consolidar 3 etapas de alfabetización: adiestramiento, enseñanza de lecto-escritura, y consolidación.

1. Adiestramiento. Etapa que comprende un total de 10 clases; 5 de las cuales buscan familiarizar a los participantes con el método y trabajan la estimulación de la expresión oral y las habilidades psicomotoras y 5 que están dirigidas al estudio de las vocales, utilizando la repetición como método de consolidación.

2. Enseñanza de lecto-escritura. Etapa que comprende un total de 42 clases. En las primeras 23 clases se enseña cada día una nueva letra o fonema, de acuerdo a la frecuencia de uso en el lenguaje cotidiano; mientras que las 19 restantes se utilizan para introducir dificultades en el idioma castellano - i.e. combinación de distintos fonemas, las reglas en la utilización de la "r" y "rr". Durante esta etapa la técnica utilizada relaciona cada letra o fonema con un número y un gráfico, para así ir presentando nuevas palabras y objetos de estudio.

3. Consolidación. Etapa que comprende un total de 13 clases, que se utilizan para reforzar lo aprendido y evaluar el resultado en cada uno de los participantes. En esta etapa, el trabajo del facilitador es consolidar los conocimientos adquiridos, fortalecer los conocimientos que necesitan mayor decisión y evaluar sistemáticamente a cada

4 Decreto Supremo N 28675 del 13 de Abril de 2006, Artículo $7^{\circ}$

5 Decreto Supremo N 28675 del 13 de abril de 2006, Artículo $2^{\circ}$ 
uno de los participantes. En su evaluación final los participantes debían escribir una carta en la que debían expresarse con coherencia y con una caligrafía legible.

Para la implementación del programa, la Dirección de Alfabetización instaló28,450 puntos de alfabetización en el país y movilizó a 46,460 facilitadores y 4,949 supervisores, los que tuvieron a su cargo 50,582 grupos y, de acuerdo con las cifras oficiales, lograron graduar a 824,101 analfabetos. La Tabla 1 presenta la distribución de puntos, recursos humanos, grupos y graduados por departamento.

\subsection{Cobertura}

De acuerdo con los datos de la Unidad de Alfabetización del Vice-Ministerio de Educación Alternativa, el PNA graduó824,101 personas analfabetas distribuidas en los 327 municipios de los 9 departamentos del país. La Tabla 2 presenta las tasas de cobertura nacional y por departamentos. Para definir el denominador de los datos de cobertura se analizaron dos fuentes de información: (1) el Censo del PNA 2007, y (2) proyecciones de los niveles de analfabetismo basadas en el Censo Nacional De Población y Vivienda 2001. El Censo del PNA 2007 identifica una población objetivo de 823,256 analfabetos, distribuidos mayoritariamente en los departamentos de $\mathrm{La} \mathrm{Paz} \mathrm{(con} \mathrm{236,} 557$ analfabetos), Cochabamba (con 161, 210 analfabetos), Santa Cruz (con 129,059 analfabetos) y Potosí (con 106,413 analfabetos). De acuerdo a estas estimaciones, la cobertura del programa a nivel nacional habría alcanzado el $100 \%$ de la población objetivo, con niveles de cobertura departamental cercanos o superiores al $100 \%$ con excepción del departamento de Tarija que habría tenido una cobertura de $83.5 \%$ - la más baja registrada a nivel departamental6. Una alternativa a las estimaciones del Censo del PNA 2007 es utilizar las proyecciones del INE de población a nivel municipal junto a proyecciones simples de la evolución de las tasas de analfabetismo basadas en las tasas observadas en el periodo intercensal 1992-20017. De acuerdo con estas estimaciones la cobertura del programa a nivel nacional habría alcanzado el $80.6 \%$ de la población objetivo, con niveles de cobertura departamental cercanos o superiores al $100 \%$ para la mayoría de los departamentos del país, exceptuando Chuquisaca, Potosí y Tarija donde la cobertura habría sido apenas superior al $60 \%$.

6 Lamentablemente, no existe información sobre los métodos utilizados por el Censo del PNA para la estimación de la población analfabeta.

7 Específicamente, las estimaciones de población analfabeta bajo esta aproximación se obtuvieron a partir del siguiente cálculo:

$$
\begin{aligned}
& N A_{2007}=\hat{\pi}_{2007} * N_{2007}
\end{aligned}
$$

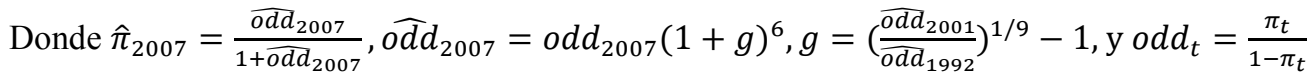


La Figura 1 muestra la distribución de los niveles de cobertura a nivel municipal para todo el país. Esta distribución muestra que a nivel municipal la tasa de cobertura varía desde valores del $65 \%$ hasta $160 \%$, aunque un $70 \%$ de los municipios se concentran en tasas de cobertura cercanas al $100 \%$. Un aspecto a considerar es la cantidad de municipios que presentan tasas de cobertura mayores al $100 \%$ en la distribución, estos valores explicarían que muchos de los participantes del programa en algunos municipios no se declararon analfabetos según el Censo del PNA-Bolivia. Por otra parte, la Figura 4 muestra la distribución de la tasa de cobertura municipal por departamento. Este análisis permite distinguir la variabilidad en la cobertura entre municipios según el departamento al que pertenecen. Mientras que la mayoría de los departamentos presentan más del 50\% de los municipios con una tasa de cobertura cercana al $100 \%$, existen algunos departamentos que muestran mayor variabilidad entre sus municipios y concentran un mayor porcentaje con tasas de cobertura menores al 100\%, como es el caso de Chuquisaca o Tarija.

\subsection{Selección}

Como en la mayoría de los programas de alfabetización de adultos, el PNA estuvo abierto a todas las personas interesadas en participar. Para caracterizar los mecanismos de autoselección utilizamos la "Encuesta de Diagnóstico Socio-cultural del Participante en el PNA" realizada por el Ministerio de Educación y Culturas8. La Tabla 3 presenta las características socio-demográficas de los participantes del PNA de acuerdo con esta encuesta. El Panel A muestra las características individuales de los participantes, resaltando la gran participación de mujeres dentro del programa. La proporción de mujeres participantes supera el $70 \%$ en la mayoría de los departamentos, alcanzando un valor de $89.3 \%$ en la ciudad de Potosí. Un caso particular de esta relación entre hombres y mujeres participantes es el departamento de Pando, en el cuál el porcentaje de mujeres alcanza simplemente un 53\%. En cuanto a las demás características individuales, la presencia mayoritaria de personas entre 36 y 55 años es evidente en la mayoría de los departamentos, al igual que el idioma castellano. El desarrollo del programa en un idioma nativo representa un porcentaje considerable en el altiplano y los valles, sin embargo en el oriente del país el castellano es el idioma en el cuál fueron alfabetizados más del $85 \%$ de los participantes. Finalmente, la mayoría de los participantes son casados y tienen hijos, lo que posiblemente se puede relacionar por el grupo etéreo mayoritario dentro del programa.

El Panel B y Panel C identifican las características de la comunidad y vivienda a la cuál pertenecen los participantes respectivamente. Note que existe una gran variabilidad en el acceso a los servicios básicos entre departamentos - i.e. los participantes del departamento de Tarija presentan un acceso a agua potable mayor al $95 \%$, mientras que en el departamento de Pando menos del 50\% de los participantes tienen acceso a este servicio. Dentro de las características de la comunidad es necesario notar el acceso a telefonía por parte de los participantes. Los porcentajes de acceso a este servicio en la comunidad son menores al $25 \%$ en la mayoría de los departamentos y se reduce aún más a nivel vivienda, con porcentajes de acceso menores a $10 \%$ en la mayoría de los departamentos. Esta es una

8 Lamentablemente, no fue possible acceder a documentaci[on sobre el diseño de la encuesta, por cuanto nuestro análisis asume que la muestra encuestada es representativa de la totalidad de participantes 
característica importante que permite identificar a los participantes del programa y las condiciones de las comunidades en las que viven. Por otra parte, es necesario notar que a pesar de que una gran mayoría de los participantes cuentan con unidades educativas dentro de su comunidad, muy pocos de ellos son colegios. Esta característica muestra una de las restricciones para aspirar a alcanzar terminar la primaria o bachillerato, una de las principales motivaciones de los participantes dentro del programa.

Finalmente, el Panel D muestra algunos indicadores sobre su participación dentro del PNABolivia. Entre los materiales entregados por el PNA-Bolivia se encuentran los lentes para lectura. Lo más importante de este punto, es notar que la cobertura de entrega de lentes por parte del PNA-Bolivia a las personas que declaran necesitar es muy bajo con valores menores al 20\%, haciendo énfasis en los resultados obtenidos en el departamento de Chuquisaca que considera simplemente un $0.8 \%$ en la cobertura de entrega. Por otra parte, se observan las principales motivaciones declaradas de participar en el programa9, entre estas se destaca el objetivo principal del programa -la aspiración de leer y escribir; y la posibilidad de ayudar a miembros de la familia en la escuela, algo muy relacionado con el porcentaje de participantes que tienen hijos, un $86.7 \%$ de los participantes a nivel nacional.

\section{Cambios en las Tendencias del Analfabetismo Atribuibles al PNA}

\subsection{Motivación}

Antes de analizar si existe un quiebre en la tendencia de la tasa de analfabetismo que puede atribuirse al PNA es conveniente analizar la magnitud del reto de erradicar el analfabetismo en Bolivia. La Tabla 7 presenta la evolución del analfabetismo en Bolivia. De acuerdo a los datos de los últimos Censos de Población y Vivienda, la incidencia del analfabetismo en Bolivia disminuyó de 36.8 en 1976 a 20.0 en 1992 a 13.3 en 2001; tendencia que muestra un aumento de la tasa de reducción anual del analfabetismo de $3.74 \%$ en el periodo intercensal 1976-1992 a 4.43\% en el periodo intercensal de 1992-2001. A partir de los datos censales es posible responder dos preguntas: ¿Cuál sería la incidencia de analfabetismo que se observaría en Bolivia el 2010 manteniendo la tendencia observada en el último periodo intercensal?, y ¿Cuál debería ser la tasa de reducción anual necesaria para alcanzar tasas de analfabetismo menores al 4\% el 2010?10. La columna (6) presenta los niveles de analfabetismo que se observarían el 2010 si se mantuvieran las tasas de reducción anual del periodo intercensal 1992-2001. Si se mantuviera la tendencia observada en el último periodo intercensal, el analfabetismo en Bolivia el 2010 estaría alrededor de 8.8\%; alrededor de 20.4 y 23.7 en Chuquisaca y Potosí respectivamente -los dos departamentos con mayor incidencia de analfabetismo, y de 5.3 y 7.2 en Santa Cruz y Beni -los dos departamentos con menor incidencia de analfabetismo. En otras palabras, si se hubieran mantenido constantes las

\footnotetext{
9 Los participantes pueden declarar más de una opción como su principal motivación de participar en el programa.

10 La UNESCO declara como "territorios libres de analfabetismo" aquellos en los que sólo se observa analfabetismo residual estimado en una tasa menor al 4\% (Canavire, 2011)
} 
tendencias observadas en el último periodo intercensal no hubiera sido posible reducir las tasas de analfabetismo por debajo del $4 \%$.

La columna (7) presenta la magnitud del esfuerzo que se debería observar para que cada uno de los departamentos sean considerado como "territorios libre de analfabetismo", i.e. la magnitud de las tasas de reducción anuales que deberían observarse en el periodo 2001-2010 para que los niveles de analfabetismo se hayan reducido por debajo del $4 \%$. Si bien a nivel nacional el esfuerzo promedio para eliminar el analfabetismo es importante - se requeriría una tasa anual de reducción 2.33 veces mayor a la observada en el periodo inter-censal, para los departamentos con mayor incidencia de analfabetismo el esfuerzo necesario para eliminar el analfabetismo es mucho más grande -se requeriría una tasa anual de reducción 4.52 y 6.38 veces más alta de la que se observó en el periodo intercensal. Por ello, esta sección utiliza los datos de la declaración de analfabetismo de la serie de encuestas de hogares 1999-2009 para examinar si efectivamente existieron dichos quiebres en la tasa natural de reducción de analfabetismo y la temporalidad de dichos quiebres permite atribuir este impacto al PNA.

\subsection{Datos}

Para analizar los cambios en la tendencia de los niveles de analfabetismo después de la implementación del PNA utilizamos la serie de encuestas de hogares producida por el Instituto Nacional de Estadística y armonizada por la Fundación ARU. La serie incluye las Encuestas de Hogares de Medición de Condiciones de Vida (MECOVI) del periodo 19992002; la En-cuesta de Ingresos y Gastos del periodo 2003-2004, y las Encuestas de Hogares de Medición de Condiciones de Vida (MECOVI) del periodo 2005-2009. La armonización de la serie intenta superar -en la medida de lo posible, no sólo las diferencias en los diseños muestrales sino también las diferencias en los diseños de contenido. Para superar las diferencias en los diseños muestrales de los diferentes encuestas se utiliza un procedimiento de post-estratificación por estratos de consumo del hogar per-cápita11. En el caso de la variable de analfabetismo no existieron cambios significativos en el diseño de contenido. En todos los años la pregunta para capturar la información fue: ¿Sabe leery escribir?.

Es importante notar que en todas las encuestas de la serie la pregunta sobre el analfabetismo de los miembros del hogar podía ser respondida por cualquier miembro del hogar presente en el momento de la entrevista. La auto-heteroevaluación de la capacidad de leer y escribir presenta serias desventajas respecto de evaluaciones directas. Por una parte, Ortega y Rodriguez (2006) argumentan que en este tipo de preguntas las personas pueden exagerar su capacidad para leer y escribir, especialmente aquellos que estén participando o recientemente hayan participado de programas de alfabetización, por lo que este tipo de datos puede sobre-estimar el impacto de dichos programas. Por otra parte, Rosnik y Weisbrot (2008) argumentan que este tipo de datos puede sub-estimar el impacto de los programas de alfabetización en el caso que los participantes de dicho programas no sean completamente analfabetos sino analfabetos funcionales, ie. en el caso que los participantes ya declaren que saben leer y escribir. Por ejemplo, si bien la tasa de analfabetismo en Estados Unidos es menor al 1\% utilizando datos de auto-heteroevaluación, al menos $20 \%$ no puede leer las instrucciones más simples de la etiqueta de una medicina (Rosnik y Weisbrot, 2008. p.3). A pesar de las limitaciones de utilizar datos de auto-heteroevaluaciones de las

11 Para mayores detalles véase Hernani-Limarino (2006) y Fundación ARU (2010) 
capacidades de lectura y escritura creemos que es importante analizar los potenciales quiebres de nivel y de tendencia atribuibles al PNA, y complementarlos con evaluaciones directas de dichas capacidades cuyo análisis se presenta en la sección 4.

\subsection{Metodología}

Sea $\pi_{\mathrm{t}}$ la tasa de analfabetismo observada en la población objetivo -generalmente la población mayor a 15 a nos de edad. La forma más simple de analizar la tendencia del analfabetismo en un periodo de tiempo determinado sería descomponer la evolución en el tiempo del analfabetismo en la suma de una tendencia temporal $(\mathrm{Tt})$ y un componente aleatorio (t). Formalmente,

$$
\pi_{t}=T_{t}+\epsilon_{t}
$$

donde la tendencia temporal puede modelarse de forma lineal, $T_{t}=\alpha_{0}+\alpha_{1} t$; cuadrática, $T_{t}=\alpha_{0}+\alpha_{1} t+\alpha_{2} t^{2}$; cúbica $T_{t}=\alpha_{0}+\alpha_{1} t+\alpha_{2} t^{2}+\alpha_{3} t^{3}$; etc. Aún cuando es posible que esta aproximación permita una correcta estimación de la tendencia en el analfabetismo, es muy probable que extrapolaciones fuera de la muestra observada generen predicciones fuera del rango posible de tasas de analfabetismo, [0,1]. Para evitar esta situación es conveniente analizar no las tasas de analfabetismo sino el logaritmo del ratio de probabilidad, i.e. $\ln \left(\frac{\pi_{t}}{1-\pi_{t}}\right)$. Nótese que, la razón de probabilidades - una medida de la mayor probabilidad de observar un analfabeto respecto de un alfabetizado varía en el rango de $\left[0=\frac{0}{1},+\infty=\frac{1}{0}\right] 12$; mientras que la transformación logarítmica permite la variación en el rango de $(-\infty ;+\infty)$. Por tanto, el modelo que utilizamos para estimar las tendencias en analfabetismo es,

$$
\ln \left(\frac{\pi_{t}}{1-\pi_{t}}\right)=T_{t}+\epsilon_{t}
$$

a partir del cual es posible deducir,

$$
\pi_{t}=\frac{e^{T_{t}+\epsilon_{t}}}{1+e^{T_{t}+\epsilon_{t}}}
$$

Es importante notar que, a diferencia del modelo de la ecuación (1), en este tipo de modelos el cambio en el tiempo de la tasa de analfabetismo $\frac{\vartheta_{\pi t}}{\vartheta_{t}}$ es variable.

Una vez definida la forma funcional para estimar la tendencia temporal de la tasa de analfabetismo, la estimación de los cambios en la tendencia temporal atribuibles al PNA "Yo Si Puedo" se realizan a partir de la introducción de una variable cualitativa que identifique el periodo de funcionamiento del programa, i.e.

12 Valores menores a 1 indican mayor probabilidad de observa un analfabeto, valores mayores a 1 indican mayor probabilidad de observar un alfabetizado, y valores iguales a 1 denotan igual probabilidad de ambos eventos. 


$$
D_{t}=\left\{\begin{array}{l}
1 \text { si } t \in \text { periodos de intervención del programa } \\
0 \text { en otro caso }
\end{array}\right\}
$$

Por ejemplo, en el caso de utilizar un modelo de tendencia lineal, i.e.

$$
\ln \left(\frac{\pi_{t}}{1-\pi_{t}}\right)=\alpha_{0}+\alpha_{1} t+\delta_{0} D_{t}+\delta_{1} D_{t} * t+\epsilon_{t}
$$

tendremos tres impactos potenciales del PNA "Yo Si Puedo"; $\delta_{0}$ reflejaría el impacto del programa sobre los niveles de analfabetismo observados, $\delta_{1}$ reflejaría el impacto del programa sobre la tendencia de la razón de probabilidades de observar un analfabeto, y $\delta_{2}$ reflejaría el impacto del programa sobre la aceleración de la razón de probabilidades de observar un analfabeto.

$$
\ln \left(\frac{\pi_{t}}{1-\pi_{t}}\right)=\alpha_{0}+\alpha_{1} t+\alpha_{2} t^{2}+\delta_{0} D_{t}+\delta_{1} D_{t} * t+\delta_{2} D_{2} * t^{2}+\epsilon_{t}
$$

A pesar de que el programa inició actividades el año 2006, la mayoría de los municipios fueron declarados libres de analfabetismo al menos un año después del inicio de actividades. Específicamente, 107 municipios fueron declarados libres de analfabetismo el año 2007, 98 municipios el año 2008 y 122 municipios el año 2009. Para probar la robustez de nuestras estimaciones a la definición del periodo de intervención utilizamos 2 diferentes especificaciones. En la primera, el periodo de intervención se define a partir de la fecha de inicio de actividades. En la segunda, el periodo de intervención se define a partir de la fecha de graduación del programa - i.e. la fecha de declaración de territorio libre de analfabetismo de los primeros municipios.

\subsection{Resultados}

La Tabla 13 presenta las estimaciones de los cambios en el nivel y la tendencia (del logaritmo de la razón de probabilidad) de la tasa de analfabetismo atribuibles a la implementación del PNA. Los paneles A y B presentan estimaciones para la población de 15 o más años de edad definiendo el periodo de intervención a partir de la fecha de inicio de actividades del programa o bien a partir de la fecha de graduación del programa en el municipio, respectivamente13. Los paneles C y D presentan estimaciones para la población de 25 o más años de edad definiendo el periodo de intervención a partir de la fecha de inicio de actividades y de la fecha de graduación del municipio, respectivamente. En todos los casos, la columna (1) presenta estimaciones de la tendencia (del logaritmo de la razón de probabilidad) de la tasa de analfabetismo. Las restantes columnas añaden a esta estimación los cambios en el nivel atribuibles a la implementación del PNA (columna 2), los cambios en la tendencia atribuibles al PNA (columna 3), y los cambios tanto en el nivel y la tendencia atribuibles al PNA (columna 4). En todas las muestras y en todas las especificaciones el

13 Como se mencionó anteriormente, a pesar de que el programa inició actividades el año 2006, la mayoría de los municipios fueron declarados libres de analfabetismo al menos un año después del inicio de actividades. Por esta razón, definimos el periodo de intervención tanto a partir de la fecha de inicio de actividades como a partir de la fecha de graduación del programa. 
efecto del PNA sobre el nivel y la tendencia de analfabetismo es estadísticamente no diferente de cero.

Un problema potencial de las especificaciones utilizadas es el nivel de analfabetismo del periodo 2003-2004. Como se mencionó antes, la encuesta de ingresos y gastos 2003-2004 tuvo marcadas diferencias con el resto de las encuestas del periodo no sólo en el diseño y asignación de la muestra sino también en cuanto al periodo de aplicación de la encuesta. Mientras que la encuesta 2003-204 se aplicó de forma continua entre los meses de noviembre de 2003 a noviembre de 2004, el resto de las encuestas utilizadas fueron encuestas puntuales aplicadas en uno de los tres últimos meses del año. Para controlar por este factor la Tabla 16 replica el análisis anterior incluyendo una variable cualitativa que controla por el nivel del analfabetismo observado en dichos años. Nótese que, si bien la variable cualitativa para el periodo 20032004 es significativa en todos los casos, el efecto del PNA sobre el nivel y la tendencia del analfabetismo continua siendo estadísticamente no diferente de cero. En otras palabras, a nivel nacional, el análisis de los datos de las encuestas de hogares para el periodo 1999-2009 no presenta evidencia que muestre un cambio ni en los niveles ni en la tendencia del analfabetismo una vez que se controla por la tendencia natural de dicho indicador.

Las Tablas 14 y 17 desagregan las estimaciones por área de residencia y sexo sin y con la inclusión de la variable de control por el nivel de analfabetismo observado en el periodo 2003-2004, respectivamente, para la población de 15 o más años de edad. Las Tablas 15 y 18 reproducen el mismo análisis para la población de 25 o más años de edad.

\section{Impacto del PNA en Lectura y Escritura: El Caso de Chuquisaca}

\subsection{Motivación}

Si bien resulta interesante utilizar datos oficiales con cobertura nacional para evaluar los resultados del PNA, existen limitaciones propias de los datos de auto-evaluación de la capacidad de los miembros del hogar de leer y escribir. Es posible no sólo que las personas sobre-estimen sus propias capacidades - en cuyo caso este tipo de evaluaciones puede sobreestimar el impacto de los programas de alfabetización; sino también que las personas que participan del programa no sean analfabetos "absolutos" sino analfabetos "funcionales", i.e. que los participantes del programa ya declaren que saben leer y escribir, en cuyo caso este tipo de evaluaciones puede sub-estimar el impacto del programa. Esta sección presenta una evaluación de las ganancias en lectura y escritura de los participantes del PNA basada en evaluaciones directas de sus capacidades de lectura y escritura.

\subsection{Datos}

Para evaluar las ganancias en las habilidades de lectura y comprensión, y comunicación escrita utilizamos los datos de la Encuesta de Evaluación del PNA "Yo Sí Puedo" (EE). La EE fue real-izada por la Fundación ARU dentro de la iniciativa Red Boliviana de Microdatos y Encuestas entre los meses de mayo y junio de 2012. La EE restringió su cobertura al departamento de Chuquisaca por razones presupuestarias. Se eligió el departamento de Chuquisaca por ser uno de los dos departamentos con mayores tasas de 
analfabetismo. Por ejemplo, si se considera las tasas de analfabetismo de la cohorte de población nacida entre 1946 y 1955, Chuquisaca presenta tasas de analfabetismo superiores a $25 \%$ en el área urbana y superiores a $60 \%$ en el area rural. La EE también restringió la población objetivo a personas de 15 a 65 años con no más de 5 años de educación formal.

La EE utilizó el CNPV como marco y un diseño de muestra complejo con dos niveles de estratificación explicita y uno implícito; y dos etapas para la selección de la muestra. La estratificación primero dividió la población en áreas urbana (municipio de Sucre) y áreas rurales (otros municipios). En las áreas urbanas se definió como unidades primarias de muestreo (UPMs) a los sectores censales, mientras que las áreas rurales se definió como UPMs a las organizaciones comunitarias. Para todas las UPMs del marco se construyeron tres sub-estratos de acuerdo a la lengua materna de la población objetivo: (1) UPM con predominio del castellano, (2) UPM con predominio del quechua, y (3) UPM con mezcla de castellano y quechua14. Asimismo, para todas las UPMs del marco se construyó la probabilidad de encontrar a una persona que pertenezca a la población objetivo; variable que fue utilizada para la primera etapa de selección de la muestra. Para maximizar la probabilidad de encontrar personas de tratamiento y control dentro de la población objetivo y minimizar los costos operativos de la encuesta, sólo se incluyó en la selección las UPMs urbanas con probabilidad superior al percentil 90 y las UPMs rurales con probabilidad superior a la probabilidad media. En la segunda etapa se seleccionaron hogares a partir de una tabla de selección aleatoria no sistemática.

Además de mediciones de capacidades básicas de alfabetización, la EE recolectó información de las características socio-demográficas (sexo, edad, lugar de nacimiento, lengua materna y lengua habitual, estado civil y acceso a educación formal); los antecedentes familiares (educación y ocupación de los hijos y de los padres); la primera y las dos últimas ocupaciones (tipo de ocupación, días y horas trabajadas regularmente, y si necesitaba leer, escribir o hacer cálculos en el trabajo); y los hábitos de lectura, escritura y cálculos (frecuencia de lectura de cartas y noticias, llenado de formularios, ayuda a los hijos con sus tareas, entre otros).

La tabla 5 presenta en detalle las características mencionadas anteriormente tanto para participantes del PNA como para no participantes de acuerdo a la EE. Se observa por ejemplo, que la mayor parte de la muestra corresponde a mujeres y con mayor énfasis en el área urbana. Aproximadamente el $50 \%$ se encuentran entre los 26 y 45 años de edad, situación que se ajusta también al estado civil de los encuestados, mayormente casados. Esta información guarda una relación importante con la situación socio-demográfica de los beneficiarios presentada en la tabla 3, lo que permite inferir la validez de la muestra. Otras características importantes a observar en el caso de alfabetización son los antecedentes familiares y las condiciones laborales actuales, se observa por ejemplo que más del $56 \%$ de los padres de los entrevistados no asistieron a la escuela, y en el área rural para los participantes del PNA llega a ser de aproximadamente $63 \%$, la tasa de inasistencia de las madres es aún mayor. Por otra parte, la media de horas trabajadas de los participantes es de 10.5 horas por día y la media de días trabajados es de 6.5 lo que implica que probablemente no exista el tiempo suficiente para completar o mejorar la educación que tienen. Para más del

14 Para construir los estratos se utilizó el método de k-medias. 
$50 \%$ de participantes y no participantes del PNA, sus ocupaciones no requieren que lean, escriban o realicen cálculos matemáticos.

De manera complementaria, se preguntó a los participantes algunas de las percepciones respecto al PNA. La tabla 6 muestra entre otros aspectos, opiniones respecto al método, facilitadores y materiales. Un aspecto a destacar es que un $50 \%$ en el área urbana y un $25 \%$ en el área rural no utilizaron video dentro el PNA, aspecto contradictorio con el método propuesto.

\subsection{Metodología}

\subsubsection{Evaluación Directa de las Dimensiones de Alfabetización}

Ciertamente, el resultado esperado de un programa de alfabetización no es que las personas (o sus familiares) declaren que ellos saben leer y escribir, sino que efectivamente las personas adquieran la capacidad de leer y entender el lenguaje escrito, y la capacidad de utilizar el lenguaje escrito para comunicarse. Por ello, es aconsejable utilizar evaluaciones directas para medir las capacidades básicas que hacen que una persona se considere como alfabetizada. Siguiendo las recomendaciones de UNESCO (1961), utilizamos la prueba descrita en el Recuadro 1 para medir dos dimensiones básicas que hacen a una persona alfabetizada: 1) Lectura y com-prensión, y 2) Escritura y comunicación. El objetivo de nuestra prueba no fue realizar una medición exhaustiva y completa de las capacidades de escritura y lectura de las personas entrevistadas como la que realiza la Encuesta Internacional de Alfabetización de Adultos15 -cuya prueba utiliza 114 tareas para categorizar a las personas en uno de cinco niveles de habilidad para cada uno de los dominios de análisis, sino hacer una medición directa y simple de la capacidad de lectura y comprensión, y comunicación escritura de los graduados del PNA. A diferencia de las mediciones exhaustivas y completas, las evaluaciones simples permiten mediciones más claras y comparables de las capacidades de lectura y comprensión y comunicación escrita (Véase Schaffner, 2005).

La primera pregunta presenta una prueba objetiva de capacidad de lectura y comprensión. En esta pregunta, el encuestador presentó al encuestado una oración de 17 palabras, y evaluó su capacidad de lectura mecánica, i.e. su capacidad de reproducir los sonidos asociados convencionalmente a cada uno de los símbolos utilizados, $y_{L C 1}$, y su capacidad de lectura comprensiva, i.e. su capacidad de separar adecuadamente las palabras de la oración y comprender el significado de cada una de ellas, $y_{L C 2}$. En ambos casos, las evaluaciones se realizaron a partir de una variable cualitativa que denota la presencia o no de dichas capacidades. Si bien es poco probable que existan errores de evaluación en el indicador de lectura mecánica, no es posible descartar que la evaluación de lectura comprensiva esté sujeta a diferencias de percepción de los encuestadores aún cuando el trabajo de capacitación haya intentado homogenizar dicha percepción. Por ello, la segunda pregunta presenta una forma más directa de evaluar la capacidad de lectura comprensiva a partir de la asociación entre una figura y un texto que describe la figura. Si bien la figura muestra claramente a una persona trabajando la tierra, el texto provee información adicional sobre quién es dicha

15 International Adult Literacy Survey (ALS) 
persona. En este caso, la evaluación de la capacidad de lectura comprensiva, $y_{L C 3}$, está asociada con el uso del nombre propio, "Luis", y de la acción que dicha persona realiza, "trabaja", para describir la figura. Finalmente, la última pregunta provee el espacio al encuestado para escribir un comentario acerca de la prueba y la encuesta donde frases u oraciones coherentes y con caligrafía legible indican la presencia de la capacidad de comunicación escritura. En este caso, se evaluó la capacidad de comunicación escrita a partir del uso de al menos un adjetivo o frase, $y_{C E 1}$; al menos un adjetivo o frase apropiado al contexto de la pregunta, $y_{C E 2} ; \mathrm{y}$ al menos una frase (verbo y adverbio) apropiado al contexto de la pregunta, $y_{C E 3}$. La Figura 5 presenta tres ejemplos de las respuestas obtenidas en cada una de estas categorías.

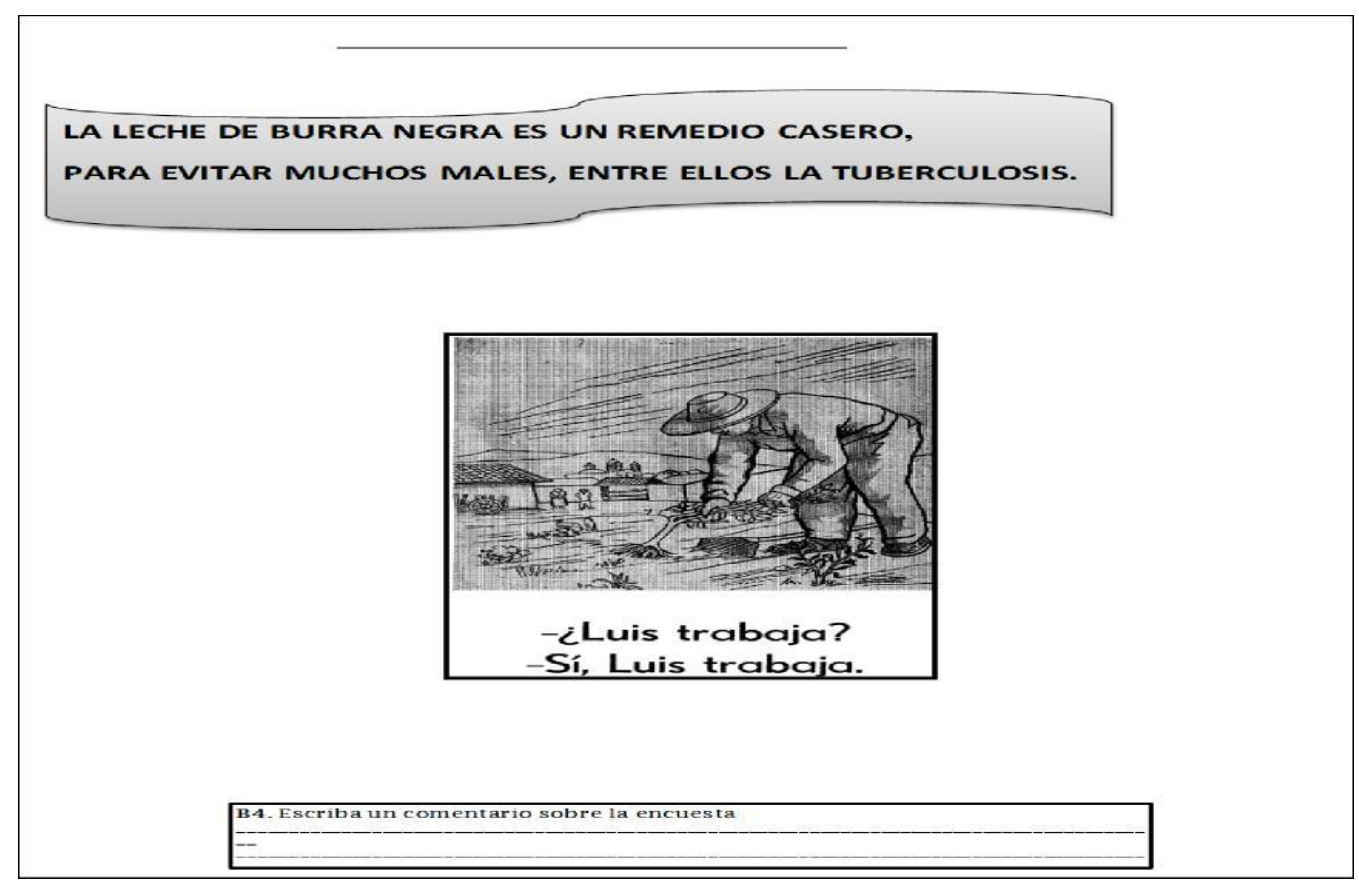

\subsubsection{Identificación del Impacto del PNA}

Una vez definidos los indicadores de resultado del programa es necesario evaluar el valor agregado del PNA "Yo Sí Puedo" en cada una de estas dimensiones. La evaluación del valor agre-gado o impacto del programa no es un problema trivial. Para analizar el problema de evaluación de impacto de manera más formal entiéndase por Wi el mecanismo de asignación al programa, i.e.

$$
W_{i}=\left\{\begin{array}{l}
1 \text { si el individuo } i \text { participa del programa } \\
0 \text { en otro caso }
\end{array}\right\}
$$

El impacto o efecto causal del programa sobre cualquiera de los indicadores de resultado $y_{i j}$ para el individuo $i$ estará dado por:

$$
\tau_{i}=\left[y_{1 i} \mid W_{i}=1\right]-\left[y_{0 i} \mid W_{i}=0\right]
$$


donde $y_{1 i}$ y $y_{0 i}$ son los resultados contrafactuales para el individuo $i$ con y sin el programa, respectivamente. El problema fundamental de la evaluación de impacto de un programa radica en que, o bien observamos el resultado cuando el individuo participa del programa, $y_{1 i}$; o bien el resultado cuando el individuo no participa del programa, $y_{0 i}$; pero bajo ninguna circunstancia observamos ambos para el mismo periodo de tiempo. Existe una variedad de soluciones planteadas por la literatura para abordar este problema. Las soluciones van desde diseños de evaluación experimentales, en los que se utiliza un mecanismo de asignación aleatorio para garantizar que no existan diferencias en el valor esperado de los resultados entre los grupos de "tratamiento" y "control" salvo aquellas ocasionadas por el hecho de que el grupo de tratamiento tuvo acceso al programa; hasta diseños cuasi-experimentales que identifican el efecto causal bajo diferentes supuestos de identificación. Dado que nuestra encuesta se realizó una vez concluido el PNA y que no se realizó una linea base que nos permitirá tener información sobre las capacidades de lectura y comprensión, y comunicación y escritura; utilizamos métodos de identificación, estimación e inferencia que asumen ausencia de factores de confusión16. El supuesto de ausencia de factores de confusión se refiere a la situación en la cuál el ajuste del valor esperado de los resultados por las diferencias en variables observables tanto en el grupo de tratamiento como en el grupo de control es suficiente para remover todo el efecto de selección no aleatoria de las personas entre los grupos de tratamiento y control, i.e. una vez que se condiciona el valor esperado de los resultados a variables observables no existen factores no observados que estén asociados ni con la asignación ni con los posibles resultados. Aunque este supuesto puede ser controversial, no existen alternativas superiores claras para el tipo de datos que utilizamos.

Es posible demostrar que bajo los supuestos de: (1) Ausencia de factores de confusión, i.e. que más allá de las covariables observadas, no existen características no observadas asociadas ni con los resultados potenciales ni con la asignación al tratamiento;

$$
\left.\left(y_{i}(0), y_{i}(1)\right) W_{i} \mid X_{i}\right)
$$

Y (2) Traslape, i.e. que para todos los posibles valores de las covariables, existe traslape en la distribución de las personas tratadas y la distribución de personas no tratadas;

$$
0<\operatorname{Pr}\left(W_{i}=1 \mid X_{i}=x\right)<1, \text { para todo } x
$$

Es posible identificar el efecto promedio de tratamiento sobre los tratados (ATT17) - i.e. el valor agregado del PNA "Yo Si Puedo" sobre los participantes, $\tau$ a partir de la diferencia de los resultados promedio de los grupos de tratamiento y control una vez que se controla por factores de confusión observables. Formalmente,

$$
\tau=E\left[y_{1 i} \mid X_{i}, W_{i}=1\right]-E\left[y_{0 i} \mid X_{i}, W_{i}=0\right]=E\left[y_{i} \mid X_{i}, W_{i}=1\right]-E\left[y_{i} \mid X_{i}, W_{i}=0\right]
$$

16 El supuesto de ausencia de factores de confusión se conoce en la literatura especializada como método "unconfoundedness".

17 Por su traducción en inglés Average Treatment Effect on the Treated. 
donde $X_{i}$ es el set de covariables observadas18. El apéndice metodológico al final del documento presenta los diferentes métodos de estimación e inferencia utilizados para la evaluación del programa.

\subsection{Impacto del PNA sobre las Capacidades de Lectura y Escritura}

Bajo los métodos de estimación e inferencia que asumen ausencia de factores de confusión, el impacto o "valor agregado" del PNA sobre las capacidades de lectura y escrita de los participantes, i.e. el efecto promedio de tratamiento sobre los tratados, puede estimarse a partir de diferencia de los resultados promedio de las evaluaciones directas entre los grupos de tratamiento y control una vez que se controla por factores de confusión observables ${ }^{17}$. Antes de analizar los resultados de dichos procedimientos es útil analizar la distribución de los resultados de las evaluaciones de lectura y escritura como las diferencias incondicionales en resultados promedio y en potenciales factores de confusión entre grupos de tratamiento y control.

\subsubsection{Diferencias Simples Incondicionales}

La Tabla 19 presenta los valores promedios de los 3 indicadores de lectura y los 3 indicadores de escritura para la muestra de participantes del PNA (grupo tratamiento) y la muestra de no participantes del PNA (grupo de control); así como las diferencias simples incondicionales entre los valores promedio de dichos grupos normalizadas por la raíz cuadrada de la suma de las varianzas19. Es importante recalcar que las diferencias simples que se presentan en estas tablas corresponden a las diferencias de los valores promedio sin controlar por potenciales factores de confusión observables y por tanto no necesariamente están relacionadas con el efecto promedio del tratamiento sobre los tratados, ie. con el valor agregado en lectura y escritura del PNA.

A nivel nacional las diferencias incondicionales entre los grupos de tratamiento y control para los indicadores de lectura no son significativamente diferentes de cero (nótese que las diferencias normalizadas de los tres indicadores analizados no llega a una desviación estándar); mientras que los indicadores de escritura son positivas y estadísticamente significativos (donde el grupo de tratamiento tiene una probabilidad mayor a dos desviaciones estándar que la del grupo de control de realizar satisfactoriamente las pruebas de escritura tanto gruesa como fina). La desagregación por área muestra diferencias

18 Véase Imbens y Wooldridge (2008) para mayores detalles.

19 La diferencia normalizada de los valores promedio se calcula a partir de la fórmula,

$$
\frac{\bar{X}_{1}-\bar{X}_{0}}{\sqrt{S_{X_{0}}^{2}-S_{X_{1}}^{2}}}
$$

donde $\bar{X}_{w}=\frac{1}{N_{w}} \sum_{i: W_{i}=w} X_{i}$ y $S_{X_{w}}^{2}=\frac{1}{N_{w}-1} \sum_{i: W_{i}=w}\left(X_{i}-\bar{X}_{w}\right)^{2}$. Estos estadísticos son mejores indicadores que estadísticos $t$-student convencionales, dado que los últimos dependen del tamaño de la muestra, y por tanto es probable que estadísticos $t$ más grandes indiquen sólo tamaños de muestra más grandes. 
normalizadas estadísticamente no significativas tanto en lectura como en escritura para el área urbana y diferencias normalizadas positivas y estadísticamente significativos sólo para algunos de los indicadores de escritura. Por sexo, las diferencias normalizadas en lectura son no significativas tanto para hombres como para mujeres, mientras que las diferencias normalizadas en escritura son positivas y estadísticamente significativas tanto para hombres como para mujeres en la mayoría de los indicadores utiliza-dos. Finalmente, la desagregación por años de educación formal (la que puede asociarse con el tipo de analfabetismo, "absoluto" para quienes presentan 2 o menos años de educación formal y "funcional" para quienes presentan más de 2 años de educación formal) muestra diferencias normalizadas no significativas en lectura para ambos grupos, y diferencias normalizadas positivas y significativas para quienes presentan más de 2 años de educación formal.

\subsubsection{Evaluando y Mejorando el Supuesto de Traslape}

Como se mencionó anteriormente, las diferencias simples no necesariamente están relacionadas con el efecto promedio del tratamiento sobre los tratados dado que es posible que existan factores de confusión tanto observables como no observables que dificulten la comparación directa del grupo de participantes del PNA y el grupo de no-participantes del PNA. Para examinar las diferencias entre los grupos de tratamiento y control la Tabla 20 presenta las diferencias en los valores promedio de las covariables más importantes para explicar la participación en el PNA - i.e. la probabilidad de recibir tratamiento. Nuevamente, para evitar problemas originados en el tamaño de la muestra presentamos no sólo los valores promedio de las covariables, sino también las diferencias normalizadas. Nótese que existen marcadas diferencias en casi todas las variables que predicen la participación en el PNA. La muestra de tratamiento tiene más edad ( +4 d.e.), mayor porcentaje de mujeres $(+6.8$ d.e. $)$, menos años de educación (-3.8 d.e.), menor porcentaje de casados en el área urbana $(-2.1$ d.e.), mayor porcentaje de gente con certificado de nacimiento (+2 d.e.), y menor probabilidad de leer direcciones en su primera ocupación (-5.5 d.e.).

La inspección de diferencias normalizadas en las covariables por estado de tratamiento es un punto de partida sensible pero generalmente no suficiente. Incluso si la distribución marginal de las covariables es similar, pueden seguir existiendo regiones en el espacio de covariables donde la densidad multivariada de las covariables en el grupo de tratamiento es cero, mientras que la densidad multivariada en las covariables del grupo de control no, o viceversa. Un en-foque complementario para evaluar la traslape en la distribución multivariada de covariables es inspeccionar los histogramas de los puntajes de probabilidad20 estimados por estado de tratamiento. La Figura 16 muestra dichos puntajes tanto para el grupo de tratamiento (Panel A) como para el grupo de control (Panel B). Nótese que, como era de esperarse, existe un desbalance de la distribución de puntajes entre el grupo de tratamiento y el grupo de control. Mientras que la distribución de puntajes de probabilidad del grupo de tratamiento está sesgada hacia 1, la distribución del grupo de control está sesgada hacia 0; con áreas sin traslape entre ambos grupos (por ejemplo, nótese que no existen puntajes de probabilidad en el grupo de tratamiento con probabilidad menor a $.05 \mathrm{y}$ que no existen puntajes de probabilidad en el grupo de control con probabilidad mayor a $.65)$.

20 Llamados también propensity scores. 
Como estamos interesados en el efecto ATT y nuestra muestra de control es más grande que nuestra muestra de tratamiento, utilizamos el procedimiento de Rubin para construir una muestra de controles con mayor traslape (Véase Rubin,2006 para mayores detalles). Primero, estimamos un nuevo puntaje de probabilidad con una especificación más flexible que incluye potencias e interacciones de las covariables relevantes (lo que incluye la edad, sexo, años de educación estado civil, área de residencia, disponibilidad de certificado de nacimiento, y el uso de lectura, escritura o cálculos en la primera o en las dos últimas ocupaciones). Luego, ordenamos las observaciones del grupo de tratamiento en forma decreciente de acuerdo con los valores del puntaje de probabilidad estimado21; para emparejar al primer individuo tratado -i.e. aquél con el valor más alto del puntaje de probabilidad estimado, con la unidad de control más cercana. Posteriormente, el segundo individuo tratado es emparejado con el segundo individuo de control más cercana, incluyendo el individuo de control que fue utilizado como pareja de la primera unidad tratada; hasta que todos los individuos tratados son emparejados con algún individuo del grupo de control, y así obtenemos una muestra emparejada de 2N1 unidades (donde N1 es el tamaño de la muestra original del grupo de tratamiento), la mitad de ellos del grupo de tratamiento y la mitad de ellos del grupo de control. Es importante notar que el procedimiento de Rubin fue utilizado no para estimar los efectos ATT por las diferencias promedio dentro de las parejas, sino para mejorar el traslape de la muestra. A partir de la muestra de emparejada es posible construir un nuevo puntaje de probabilidad y, a partir de él reexaminar el traslape de la muestra. La Figura 17 muestra los nuevos puntajes de probabilidad tanto para el grupo de tratamiento (Panel A) como para el grupo de control (Panel B). Nótese que, existe una mejora sustancial en el traslape de la muestra aún cuando todavía existen áreas sin traslape entre ambos grupos (por ejemplo, nótese que no existen puntajes de probabilidad en el grupo de tratamiento con probabilidad menor a 0.40 y que no existen puntajes de probabilidad en el grupo de control con probabilidad mayor a 0.60). Para analizar la sensibilidad de los resultados al uso de éste procedimiento, a continuación se presentan la estimación e inferencia del valor agregado del PNA utilizando tanto la muestra completa como la muestra emparejada.

\subsubsection{Impacto del PNA}

La Tabla 22 presenta estimaciones del impacto del PNA sobre los participantes, i.e. el efecto tratamiento sobre los tratados, para 3 indicadores de lectura y 3 indicadores de escritura a partir de 7 métodos de estimación e inferencia diferentes: regresión; ponderación (weighting); estratificación (blocking); emparejamiento (matching); y la combinación de los últimos tres con el primero: regresión y ponderación; regresión y estratificación; y regresión y emparejamiento. Es importante notar que los 7 métodos utilizados asumen que, una vez que se controla por aquellas variables observables que ayudan a predecir la participación en el programa, no existen características no observadas asociadas ni con los resultados potenciales ni con la asignación al tratamiento. La diferencia entre los diferentes métodos

21 La racionalidad para esto es que entre las unidades con grandes valores del puntaje de probabilidad hay relativamente más individuos tratados que individuos de control, y por lo tanto, las observaciones tratadas con valores altos del puntaje de probabilidad son relativamente mas difíciles de emparejar 
radica en los mecanismos que utilizan para controlar por las variables observables y realizar las comparaciones entre los grupos de tratamiento y control (Para mayores detalles véase Imbens(2006) o el apéndice metodológico). El panel A presenta estimaciones utilizando la muestra completa, i.e. 279 individuos tratados y 635 individuos de control; mientras que el panel B presenta estimaciones utilizando la muestra emparejada, i.e. 279 individuos tratados y 279 individuos de control. Para cada uno de los indicadores de resultado y métodos utilizados se presentan 3 estimaciones: el efecto promedio del tratamiento sobre los tratados (SATT), i.e. el aumento promedio en la probabilidad de realizar satisfactoriamente la prueba correspondiente que puede ser atribuido al PNA; la desviación estándar, i.e. la distancia promedio de los efecto de tratamiento observados respecto del efecto promedio; y el indicador de significancia estadística $t$-student 22 .

Para el primer indicador de lectura nuestras estimaciones muestran un impacto estadísticamente significativo del PNA de 8 a 10 puntos porcentuales, tanto en las estimaciones utilizando la muestra completa como en las estimaciones que utilizan la muestra emparejada. En otras palabras, se evidencia un aumento de 8 a 10 puntos porcentuales en la probabilidad de los participantes de leer mecánicamente el texto presentado. Sin embargo, para los dos indicadores de lectura comprensiva nuestras estimaciones muestran un impacto estadísticamente no diferente de cero tanto en la muestra completa como en la muestra pareada. Nótese que en ninguno de los métodos utilizados los indicadores de significancia estadística alcanzan el valor necesario. Por otra parte, los tres indicadores de escritura analizados presentan valores estadísticamente significativos. Para el indicador de escritura más grueso -aquel que considera que una persona escribe si utilizó al menos un adjetivo para realizar un comentario, las estimaciones arrojan un impacto de 10 a 16 puntos porcentuales; para el indicador de escritura intermedio - aquel que evalúa la capacidad de escribir si utilizó al menos, las estimaciones arrojan un impacto de 7 a 10 puntos porcentuales; mientras que para el indicador de escritura fina - aquel que evalúa la capacidad de escribir a partir del uso de al menos un verbo y un adverbio, las estimaciones muestran un impacto del PNA de 6 a 9 puntos porcentuales. En síntesis, el conjunto de estimaciones muestra un impacto estadísticamente significativo aunque cuantitativamente pequeño en el indicador de lectura mecánica, ningún impacto estadísticamente diferente de cero en los dos indicadores de lectura comprensiva; y un impacto estadísticamente significativo aunque cuantitativamente pequeño en los tres indicadores de comunicación escrita.

\subsubsection{Midiendo el Sesgo de Selección a partir de Pseudo-resultados}

Es importante notar que nuestra prueba incluyó una última pregunta que pedia al entrevistado la realización de las 4 operaciones básicas de cálculo (suma, resta, multiplicación y división), para evaluar la capacidad numérica ${ }_{Y C N}$. Si bien la capacidad de realizar cálculos no fue parte de la primera etapa del programa, utilizamos esta medición para realizar pruebas de consistencia de nuestro análisis de evaluación. De la misma manera,

22 El indicador t-student fue alculado como la razón del efecto promedio del tratamiento sobre los tratados y su desviación estándar. Valores mayores a 1.96 son comúnmente utilizados como estadísticamente significativos, asumiendo que la distribución del indicaor de significancia estadística puede ser aproximada por una distribución normal estándar. 
incorporamos al análisis las características de las personas en actividades diarias relacionadas con la lectura y escritura, considerando la ayuda que reciben al momento de leer una carta, leen las noticias o formularios. La Tabla 23 muestra el efecto de tratamiento medio obtenido para los pseudo-outcomes descritos.

\section{Conclusiones}

Este documento evalúa la experiencia boliviana con el Programa Nacional de Alfabetización (PNA) "Yo Sí Puedo" - un método de alfabetización masiva desarrollado por la pedagoga cubana Leonela Reyes que utiliza fundamentalmente métodos audiovisuales (17 videos) para desarrollar habilidades de lectura y compresión y escritura y comunicación en 65 clases presenciales. Dos son los objetivos principales de nuestra evaluación. Primero, analizar las tendencias nacionales de analfabetismo y las de grupos particulares para investigar si es posible identificar cambios en los niveles de analfabetismo o cambios en los cambios en el tiempo que puedan atribuirse al PNA "Yo Sí Puedo"; y si dichos cambios fueron suficientes para reducir las tasas de analfabetismo por debajo de 4\% -nivel que utiliza la UNESCO para clasificar a los territorios como libres de analfabetismo. Segundo, analizar el valor agregado del PNA - el efecto tratamiento sobre los tratados, sobre indicadores de resultado relacionados con la habilidad de lectura mecánica, lectura comprensiva, y escritura y comunicación utilizando métodos de evaluación que asumen la ausencia de factores de confusión.

Para cumplir con el primer objetivo, utilizamos la serie armonizada de encuestas de hogares del periodo 1999-2009 para estimar tanto cambios en el nivel como cambios en la tendencia del analfabetismo que puedan ser asociados con el PNA "Yo Sí Puedo". Nuestros resultados muestran que, tanto a nivel nacional como para casi todos los grupos estudiados, los efectos del PNA son no solo cuantitativamente pequeños sino que estadísticamente no significativos.

Para cumplir con el segundo objetivo, utilizamos una Encuesta de Evaluación del PNA "Yo Si Puedo" para evaluar directa las habilidades básicas esperadas en una persona alfabetizada - tanto en lectura y comprensión como en escritura y comunicación, y para recogió información sobre potenciales factores de confusión del efecto del PNA sobre dichos resultados como características socio-demográficas, laborales, y hábitos de lectura y escritura. La encuesta recopiló información para una muestra representativa de participantes y no participantes del PNA "Yo Sí Puedo" de 15 a 65 años de edad con no más de 5 años de educación formales en el departamento de Chuquisaca. Los resultados obtenidos sugieren que el programa no tuvo ningún impacto en el valor agregado sobre las habilidades de lectura, mostrando que el efecto de tratamiento medio no es estadísticamente significativo. Por otro lado, existe un leve impacto en las habilidades de escritura, el cuál se va reduciendo a medida que la complejidad del test aumente. Sin embargo, este impacto sugiere que simplemente 10 a 15 personas de cada 100 que participaron en el PNA podría aprobar las pruebas de escritura. 


\section{Referencias}

[1] Abadie, A. and G. Imbens (2002). Simple and bias-corrected matching estimators for average treatment effects. National Bureau of Economic Research.

[2] Abadie, A. and G. Imbens (2008). Bias Corrected Matching Estimators for Average Treatment Effects. Unpublish manuscript, Harvard University.

[3] Abadzi, H. (1994). What we know about acquisition of adult literacy: is there hope? Taylor \& Francis.

[4] Abadzi, H. (2003). Improving adult literacy outcomes: lessons from cognitive research for developing countries. World Bank Publications

[5] Canavire, V.B. (2011). Educaci'фn para adultos en Am'erica Latina: programa de alfabet-izaci“øn" Yo, S'ı Puedo". Instituto de Investigaciones en Educación de la Universidad Veracruzana

[6] Canfux, J. and Liendo, T. (2008). Evaluación Del Impacto Del Programa Nacional de Alfabetización de Bolivia. Instituto Internacional de Investigación Educativa para la Integración Convenio Andrés Bello Ministerio de Educación y Cultura de Bolivia, Editorial Campos Iris, La Paz, Bolivia

[7] Canfux, J., Liendo, T. and Mora, D., (2008). Experiencias sobre el Programa Nacional de Alfabetización en Bolivia. Instituto Internacional de Investigación Educativa para la Integración Convenio Andrés Bello Ministerio de Educación y Cultura de Bolivia, La Paz, Bolivia

[8] Chowdhury, K.P. and World Bank.(1995). Literacy and primary education. World Bank, Human Resources Development and Operations Policy

[9] Rep'ublica de Bolivia.(2006). Decreto Supremo No 28675.

[10] Horvitz, D., and D. Thompson (1952). A genralization of Sampling Without Replacement from a Finite Universe, Journal of the American Statistical Association,47,663-685.

[11] Imbens, Guido and Jeffrey Wooldridge (2008). Recent Developments in the Econometrics of Program Evaluation IZA Discussion Paper NO.3640

[12] Mora, D.and Canfux, J. (2009). Alfabetización y Emancipación. Memorias y reflexiones sobre el proceso de lafabetización en Bolivia. Instituto Internacional de Investigación Educativa para la Integración Convenio Andrés Bello Ministerio de Educación y Cul-tura de Bolivia, Editorial Ipasme, La Paz, Bolivia

[13] Neijs, K. (1961). Las cartillas de alfabetización: Preparación, evaluación y empleo, Un-esco.

[14] Ortega, D. and Rodr'iguez, F. and Miguel, E. (2006). Freed from illiteracy? A closer look at Venezuela?s Robinson Literacy Campaign Wesleyan Economics Working Papers

[15] Romain, R.I. and Armstrong, L.A. (1987). Review of World Bank operations in nonformal education and training, World Bank, Education and Training Dept., Policy Division

[16] Weisbrot, M. and Rosnick, D. (2008). ?Illiteracy?Revisited: What Ortega and Rodr'iguez Read in the Household Survey, Center for Economic and Policy Research (CEPR)

[17] Schaffner, J. (2005). Subjective and objective measures of literacy: implications for current results-oriented development initiatives, International Journal of Educational Development 
[18] Rubin, D. (1973). The use of Matched Sampling and Regression Adjustments to Remove Bias in Observational Studies, Biometrics, 29, 185-203

[19] Rubin, D. (2006). Matched Sampling for Causal Effects, Cambridge University Press, Cambridge, UK.

[20] Wooldridge, J. (2007). Inverse Probability Weighted M-Estimators for General Missing Data Problems, Journal of Econometrics. 


\section{A Estimación e Inferencia}

La estimación del SATT bajo el supuesto de ausencia de factores de confusión puede ser mediante diferentes métodos, entre los que se pueden destacar la regresión, emparejamiento mediante la utilización del propensity score, la estratificación y la combinación de todas ellas. En este documento realizamos ocho diferentes estimaciones para poder observar la sensibilidad de los resultados a la elección del método ${ }^{8}$. Los métodos utilizados son los siguientes:

1. Diferencias Simples. Primero, es usualmente informativo calcular la diferencia simple de los resultados promedios entre las unidades de tratamiento, $\overline{Y_{1}}$, y unidades de control, $\bar{Y}_{0}$.

$$
\hat{\tau}=\overline{Y_{1}}-\overline{Y_{0}}
$$

2. Regresión. El enfoque de regresión para la estimación de ATT define las funciones de regresión para cada resultado potencial. En el caso más simple, las medias condicionales son asumidas como lineales en los parámetros, i.e.

$$
\mu_{w}(x)=E\left[Y_{i}(w) \mid X_{i}=x\right]=\alpha_{w}+\beta^{\prime}{ }_{w}(x-\varphi X) \text { para } w \in\{0,1\}
$$

Dados estimadores consistentes, $\hat{\mu}_{0}(x)$ y $\hat{\mu}_{1}(x)$, un estimador consistente del ATT estará dado por:

$$
\hat{\mu}=\frac{1}{N_{1}} \sum_{i=1}^{N_{1}}\left(\hat{\mu}_{1}(x)-\hat{\mu}_{0}(x)\right)
$$

Note que la regresión por mínimos cuadrados,

$$
Y_{i}=\alpha+\tau W_{i}+\beta X_{i}+\gamma\left(X_{i}-\bar{X}_{i}\right) W_{i}+\varepsilon_{i}
$$

brinda un estimador consistente del ATT, $\hat{\tau}$; y su varianza, $\hat{\sigma}_{\tau}$. Note que la interacción de las covariables con el indicador de tratamiento está basado en la desviación a partir de los valores promedio de las covariables para los tratados por esto $\hat{\tau}$ estima el SATT - no el efecto promedio de tratamiento de la muestra (SATE, por sus siglas en inglés).

3. Ponderación. La estimación del propensity score, $\hat{e}\left(X_{i}\right)$, puede ser utilizado de diferentes maneras. Un método, propuesto por Horvitz y Thompson (1952), utiliza el propensity score para ponderar las observaciones con la finalidad de estimar el ATT, proporción de los tratados en la muestra. Siguiendo a Wooldridge (2007),

$$
\hat{\tau}=\frac{1}{N} \sum_{i=1}^{N} \frac{\left[W_{i}-\hat{e}\left(X_{i}\right)\right]}{\hat{\rho}\left[1-\hat{e}\left(X_{i}\right)\right]} Y_{i}=\frac{1}{N} \sum_{i=1}^{N} k_{i}
$$


donde $\hat{\rho}=(N 1 / N)$ es la desviación estándar asintótica, ${ }^{\wedge} \sigma_{\tau}$, estará dada por:

$$
\widehat{\sigma}_{\tau}=\left(\frac{1}{N} \sum_{i=1}^{N} \hat{u}_{i}^{2}\right)^{1 / 2}
$$

donde $\hat{u}_{i}$ son los residuos MCO de la regresión de $\hat{k}_{i}$ en 1 y el score de $\hat{e}_{i}$.

4. Bloques. Otra forma de utilizar el propensity score estimado es crear bloques o estratos, estimar el ATT dentro el estrato como la diferencia en resultados promedios entre tratados y controles, y estimar el ATT total como el promedio ponderado de las estimaciones dentro del estrato - con los ponderadores iguales al porcentaje de las unidades tratadas en cada estrato. Formalmente, sean $0=c_{0}<c_{1}<\cdots<c_{j}=1$ valores límites que definen los bloques,

$$
B_{I J}=\left\{\begin{array}{l}
1 \text { si } c_{j-1} \leq e\left(X_{i}\right)<c_{j} \\
0 \text { en otro caso }
\end{array}\right\}
$$

Las estimaciones dentro del estrato del efecto ATT están dadas por las simples diferencias en los resultados promedio.

$$
\widehat{\tau_{J}}=\overline{Y_{J 1}}-\overline{Y_{J 0}}
$$

La racionalidad detrás de esta estrategia es que, si $\mathrm{J}$ es lo suficientemente grande, entonces las diferencias $c j-c j-1$ son pequeñas, existirá una pequeña variación en el propensity score dentro del estrato. Por lo tanto, podemos analizar la información como si, dentro del estrato, la información fuera generada por un experimento completamente aleatorio con probabilidades de asignación constantes dentro del estrato pero variando entre estratos. El efecto promedio de tratamiento es estimado como el promedio ponderado de las estimaciones dentro del estrato con ponderaciones iguales a la proporción de las unidades de tratamiento en cada estrato.

$$
\hat{\tau}=\sum_{j=1}^{Y} \hat{\tau}_{j} \frac{N_{j 1}}{N_{1}}
$$

La desviación estándar es luego estimada como:

$$
\sigma_{\tau}=\left(\sum_{j=1}^{J}\left(\frac{N_{j 1}}{N_{1}}\right)^{2}\left(\widehat{V}_{0 j}-\widehat{V}_{1 j}\right)\right)^{1 / 2}
$$

5. Método de Pareo. El método de pareo empareja todas las unidades de tratamiento a sus controles más cercanos. Seguimos Abadie y Imbens (2006) y conducir el emparejamiento, con reemplazo, en todas las covariables, ponderado por la matriz diagonal con la inversa de las varianzas en la diagonal. Más formalmente, sea $l_{m}(i)$ el índice de la m-ésima unidad más cercana en el grupo de control a la unidad $i$ en el grupo de tratamiento - en términos de la medida de la distancia basada en la norma $\|$.$\| . Formalmente, l_{m}(i)$ satisface: 


$$
\begin{gathered}
W_{\operatorname{lm}(i)} \neq W_{i} \\
\sum_{l: W_{j} \neq W_{i}}\left\{\left\|X_{l}-X_{i}\right\| \leq\left\|X_{l_{m}(i)}-X_{i}\right\|\right\}=m
\end{gathered}
$$

Sea $J_{m}^{i}=\left\{l_{1}(i), l_{2}(i), \ldots, l_{M}(i)\right\} \subset\left\{1,2, \ldots, N_{0}\right\}$ lo que denota el set de índices de los emparejamientos M para la unidad i. Entonces el estimador de pareo del efecto ATT estará dado por:

$$
\hat{\tau}=\frac{1}{N_{1}} \sum_{i: W_{t}=1}\left[Y_{i}(1)-\hat{Y}_{i}(0)\right]
$$

donde:

$$
\widehat{Y}_{\iota}(0)=\frac{1}{M} \sum_{j \epsilon J_{M}(i)} Y_{j} \text { para cada } W_{i}=1
$$

\section{Ponderación y Regresión}

Basado en las regresiones de mínimos cuadrados ponderados,

$$
Y_{i}=\alpha+\tau W_{i}+\beta X_{i}+\varepsilon_{i}
$$

con ponderadores $\lambda_{i}=\sqrt{W_{i}+\left(1-W_{i}\right) \frac{\hat{e}\left(X_{i}\right)}{1-\hat{e}\left(X_{i}\right)}}$

7. Bloques y Regresión. Basado en el mismo estrato (bloques) como el quinto estimador donde la regresión lineal es utilizada para estimar el efecto promedio dentro de los bloques.

Imbens y Wooldridge (2008) argumentan que, con un modesto número de estratos, este estimador es considerablemente más flexible y robusto que el método de bloques solo o la regresión sola.

8. Método de Pareo y Regresión. Abadie y Imbens (2002) muestra que cuando el emparejamiento no es exacto, el estimador de pareo será sesgado en muestras finitas. Como Rubin(1973) y Abadie y Imbens(2002) han mostrado, es posible reducir el sesgo utilizando métodos de regresión. Dada la función de regresión estimada $\hat{\mu}_{w}(x)=\widehat{\alpha}+\widehat{\beta}^{\prime}{ }_{w} x$, el estimador de emparejamiento corregido del sesgo estará dado por:

$$
\hat{\tau}=\frac{1}{N_{1}} \sum_{i: W_{t}=1}\left[Y_{i}(1)-\hat{Y}_{i}(0)\right]
$$

donde:

$$
\widehat{Y}_{l}(0)=\frac{1}{M} \sum_{j \epsilon J_{M}(i)}\left(Y_{j}+\left(\hat{\mu}_{0}\left(X_{i}\right)-\hat{\mu}_{0}\left(X_{j}\right)\right) \text { para cada } W_{i}=1\right.
$$




\section{B Tablas y Figuras}

Tabla 1: Número de Supervisores, Facilitadores, Puntos, Grupos, Graduados e Indicadores de Tratamiento Nacional y por Departamento (Personas)

\begin{tabular}{l|rrrrrrr} 
& $\begin{array}{c}\text { Facilitadores } \\
\text { por } \\
\text { Supervisor }\end{array}$ & $\begin{array}{c}\text { Puntos por } \\
\text { Facilitador }\end{array}$ & $\begin{array}{c}\text { Grupos por } \\
\text { Punto }\end{array}$ & $\begin{array}{c}\text { Graduados por } \\
\text { Grupo }\end{array}$ & $\begin{array}{c}\text { Graduados por } \\
\text { Punto }\end{array}$ & $\begin{array}{c}\text { Graduados por } \\
\text { Facilitador }\end{array}$ & $\begin{array}{c}\text { Graduados por } \\
\text { Supervisor }\end{array}$ \\
\hline Bolivia & 9.39 & 0.61 & 1.78 & 16.29 & 28.97 & 17.74 & 166.52 \\
\hline Chuquisaca & 14.25 & 0.83 & 1.68 & 18.51 & 31.02 & 25.67 & 365.80 \\
La Paz & 12.27 & 0.38 & 2.73 & 15.35 & 41.95 & 15.74 & 193.16 \\
Cochabamba & 9.64 & 0.65 & 1.72 & 18.07 & 30.99 & 20.07 & 193.35 \\
Oruro & 7.89 & 0.58 & 1.74 & 14.67 & 25.50 & 14.67 & 115.71 \\
Potosi & 7.38 & 0.63 & 1.67 & 17.59 & 29.30 & 18.36 & 135.48 \\
Tarija & 10.14 & 0.63 & 1.67 & 15.50 & 25.96 & 16.26 & 164.92 \\
Santa Cruz & 7.29 & 0.85 & 1.35 & 15.18 & 20.50 & 17.46 & 127.27 \\
Beni & 7.95 & 0.92 & 1.11 & 15.32 & 16.95 & 15.63 & 124.32 \\
Pando & 5.25 & 0.91 & 1.43 & 10.88 & 15.59 & 14.22 & 74.69 \\
\hline \hline
\end{tabular}

Tabla 2: Número de Graduados, Población Objetivo y Tasas de Cobertura del PNA-Bolivia Nacional y por Departamento según Fuente de Información

Panel A. Censo PNA $2007 \quad$ Panel B. Proyección 2007*

\begin{tabular}{|c|c|c|c|}
\hline & $\begin{array}{c}\text { Número de Graduados** } \\
\text { (Personas) }\end{array}$ & Tasa de Cobertura $(\%)$ & Tasa de Cobertura (\%) \\
\hline Bolivia & 824,101 & 100.1 & 80.6 \\
\hline Chuquisaca & 79,378 & 98.5 & 60.4 \\
\hline $\mathrm{LaPaz}$ & 233,140 & 98.6 & 97.4 \\
\hline Cochabamba & 162,411 & 100.7 & 80.6 \\
\hline Oruro & 32,514 & 106.1 & 88.7 \\
\hline Potosi & 113,394 & 106.6 & 62.3 \\
\hline Tarija & 35,457 & 83.5 & 67.4 \\
\hline Santa Cruz & 131,475 & 101.9 & 91.7 \\
\hline Beni & 31,328 & 99.6 & 103.1 \\
\hline Pando & 5,004 & 102.4 & 102.9 \\
\hline
\end{tabular}

Fuente: Elaboración de los autores en base a información de la Unidad de Alfabetización y las tasas de alfabetización de los CNPV 1992 Y 2001.

* Proyección realizada en base a las tasas de analfabetismo del CNPV 92 Y 2001

** En base a información de la Unidad de Alfabetización del Viceministerio de Educación Alternativa y Especial. 
Tabla 3: Características Socio-Demográficas de los Beneficiarios según Departamento

\begin{tabular}{|c|c|c|c|c|c|c|c|c|c|c|}
\hline & Chuquisaca & La Paz & Cochabamba & Oruro & Potosí & Tarija & $\begin{array}{l}\text { Santa } \\
\text { Cruz }\end{array}$ & Beni & Pando & Bolivia \\
\hline Porcentaje & 12.8 & 13.8 & 13.4 & 12.5 & 7.7 & 9.3 & 14.1 & 7.3 & 9.2 & 100 \\
\hline \multicolumn{11}{|c|}{ Panel A. Características Individuales } \\
\hline \multicolumn{11}{|l|}{ Sexo } \\
\hline Hombre & 25.0 & 27.6 & 21.6 & 19.6 & 10.7 & 23.1 & 20.2 & 23.5 & 47.0 & 24.2 \\
\hline Mujer & 75.0 & 72.4 & 78.4 & 80.4 & 89.3 & 76.9 & 79.8 & 76.1 & 53.0 & 75.8 \\
\hline \multicolumn{11}{|l|}{ Edad } \\
\hline 15 a 25 & 18.9 & 3.6 & 6.0 & 6.2 & 11.3 & 18.0 & 9.9 & 5.8 & 33.3 & 11. \\
\hline 26 a 35 & 17.2 & 14.4 & 19.0 & 16.8 & 17.2 & 14.8 & 22.0 & 10.9 & 18.3 & 17.1 \\
\hline 36 a 45 & 20.3 & 18.7 & 24.8 & 23.0 & 21.4 & 20.4 & 25.8 & 24.2 & 18.0 & 22.0 \\
\hline 46 a 55 & 16.2 & 23.5 & 23.7 & 22.0 & 26.9 & 20.4 & 21.3 & 31.7 & 13.4 & 21.8 \\
\hline 56 a 65 & 11.9 & 20.2 & 16.6 & 18.0 & 12.9 & 15.1 & 11.5 & 19.1 & 11.2 & 15.2 \\
\hline 66 a 75 & 6.8 & 14.0 & 6.2 & 10.0 & 4.5 & 5.1 & 4.8 & 5.1 & 3.0 & 7.0 \\
\hline Mayor a 76 & 8.6 & 5.5 & 3.7 & 3.8 & 5.5 & 5.4 & 4.3 & 3.1 & 2.5 & 4.8 \\
\hline \multicolumn{11}{|l|}{ Idioma } \\
\hline Quechua & 44.5 & 4.0 & 29.7 & 17.8 & 32.4 & 2.7 & 5.5 & 2.0 & 0.0 & 16.1 \\
\hline Aymara & 0.0 & 24.0 & 1.9 & 18.8 & 2.6 & 0.5 & 1.6 & 0.0 & 0.5 & 6.4 \\
\hline Castellano & 55.5 & 70.2 & 67.5 & 61.7 & 65.0 & 89.8 & 79.4 & 95.2 & 87.4 & 73.0 \\
\hline Guarani & 0.0 & 0.4 & 0.0 & 0.4 & 0.0 & 5.6 & 6.9 & 0.7 & 0.3 & 1.7 \\
\hline Extranjero & 0.0 & 0.4 & 0.0 & 0.0 & 0.0 & 0.0 & 0.2 & 0.0 & 8.5 & 0.9 \\
\hline Otro & 0.0 & 0.0 & 0.0 & 1.0 & 0.0 & 0.0 & 5.9 & 0.3 & 2.5 & 1.2 \\
\hline NS/NR & 0.0 & 1.0 & 0.9 & 0.3 & 0.0 & 1.4 & 0.5 & 1.8 & 0.8 & 0.7 \\
\hline \multicolumn{11}{|l|}{ Estado Civil } \\
\hline Solter(a) & 30.1 & 9.6 & 12.5 & 12.4 & 14.2 & 30.4 & 18.3 & 14.7 & 35.8 & 19.3 \\
\hline Casado(a) & 47.5 & 65.6 & 62.5 & 60.3 & 65.4 & 37.6 & 45.3 & 40.3 & 22.1 & 50.9 \\
\hline Conviviente & 10.4 & 12.0 & 11.0 & 9.0 & 6.1 & 19.1 & 26.3 & 29.7 & 37.2 & 17.1 \\
\hline Divorciado(a) & 1.6 & 2.5 & 2.2 & 2.4 & 1.3 & 1.6 & 2.3 & 2.4 & 1.1 & 2.0 \\
\hline Viudo(a) & 9.6 & 9.6 & 10.8 & 15.6 & 12.3 & 10.5 & 7.5 & 10.2 & 2.2 & 9.9 \\
\hline $\mathrm{NS} / \mathrm{NR}$ & 0.8 & 0.7 & 1.0 & 0.3 & 0.7 & 0.8 & 0.3 & 2.7 & 1.6 & 0.8 \\
\hline \multicolumn{11}{|l|}{ Hijos } \\
\hline No & 18.8 & 0.2 & 8.0 & 14.0 & 10.4 & 19.9 & 9.1 & 4.8 & 27.0 & 12.0 \\
\hline $\mathrm{Si}$ & 81.3 & 90.5 & 92.0 & 86.0 & 89.6 & 80.1 & 90.9 & 95.2 & 73.0 & 86.7 \\
\hline NS/NR & 0.0 & 8.6 & 0.0 & 0.0 & 0.0 & 0.0 & 0.0 & 0.0 & 0.0 & 0.5 \\
\hline
\end{tabular}




\begin{tabular}{|c|c|c|c|c|c|c|c|c|c|c|}
\hline Ninguno & 3.3 & 0.7 & 0.7 & 2.2 & 0.6 & 3.2 & 1.4 & 1.7 & 0.3 & 1.6 \\
\hline Inicial & 6.4 & 3.1 & 5.0 & 3.4 & 3.2 & 4.3 & 4.3 & 4.8 & 4.6 & 4.4 \\
\hline Primaria & 39.1 & 33.8 & 40.1 & 25.9 & 36.2 & 41.4 & 38.7 & 36.9 & 31.1 & 35.9 \\
\hline Secundaria & 22.9 & 42.9 & 36.0 & 45.1 & 32.4 & 21.2 & 40.0 & 44.7 & 22.4 & 34.7 \\
\hline Superior & 1.4 & 2.7 & 3.7 & 5.2 & 9.4 & 5.4 & 2.5 & 2.0 & 0.8 & 3.5 \\
\hline $\mathrm{NS} / \mathrm{NR}$ & 26.9 & 16.8 & 14.5 & 18.2 & 18.2 & 24.5 & 13.1 & 9.9 & 40.8 & 19.9 \\
\hline \multicolumn{11}{|l|}{ Situación Ocupacional } \\
\hline Desocupado & 50.4 & 0.5 & 45.7 & 39.1 & 53.7 & 43.5 & 38.5 & 45.4 & 44.5 & 38.6 \\
\hline Ocupado & 44.5 & 68.7 & 54.3 & 59.9 & 46.3 & 54.3 & 60.4 & 54.6 & 54.4 & 56.0 \\
\hline $\mathrm{NS} / \mathrm{NR}$ & 5.1 & 30.8 & 0.0 & 1.0 & 0.0 & 2.2 & 1.1 & 0.0 & 1.1 & 5.4 \\
\hline \multicolumn{11}{|c|}{$\begin{array}{l}\text { Situación Ocupacional de la } \\
\text { Pareja }\end{array}$} \\
\hline Desocupado & 18.2 & 0.7 & 19.2 & 22.0 & 15.5 & 13.4 & 11.0 & 10.9 & 20.8 & 14.4 \\
\hline Ocupado & 49.6 & 54.5 & 57.1 & 62.3 & 58.9 & 51.1 & 65.2 & 66.6 & 50.3 & 57.2 \\
\hline NS/NR & 32.2 & 44.8 & 23.7 & 15.7 & 25.6 & 35.5 & 23.8 & 22.5 & 28.9 & 28.4 \\
\hline \multicolumn{11}{|c|}{$\begin{array}{l}\text { Actividades en Tiempo } \\
\text { Libre* }\end{array}$} \\
\hline Descansa en la casa & 43.2 & 36.9 & 48.5 & 47.3 & 31.7 & 54.0 & 47.6 & 61.8 & 66.4 & 47.8 \\
\hline Labores de la casa & 63.9 & 57.8 & 66.4 & 50.5 & 57.3 & 59.9 & 67.7 & 65.5 & 70.2 & 62.1 \\
\hline Conversa con amigos & 29.5 & 27.8 & 39.2 & 24.8 & 15.2 & 36.3 & 45.3 & 51.2 & 66.4 & 36.7 \\
\hline Trabaja en el campo & 48.8 & 43.6 & 44.4 & 39.1 & 47.2 & 36.3 & 34.6 & 19.5 & 41.8 & 40.2 \\
\hline Habla con familiares & 60.5 & 46.9 & 55.8 & 53.7 & 47.2 & 54.8 & 65.4 & 63.5 & 66.4 & 57.0 \\
\hline Ve televisión & 24.4 & 18.9 & 36.2 & 19.0 & 30.4 & 26.6 & 41.0 & 42.7 & 28.7 & 29.3 \\
\hline Escucha radio & 49.6 & 39.1 & 42.7 & 26.1 & 44.7 & 53.2 & 46.9 & 46.4 & 56.0 & 44.2 \\
\hline \multicolumn{11}{|c|}{ Panel B. Características de la Comunidad } \\
\hline \multicolumn{11}{|l|}{ Servicios Básicos } \\
\hline Agua & 87.1 & 84.0 & 80.8 & 85.2 & 95.8 & 90.6 & 87.9 & 68.9 & 49.5 & 81.9 \\
\hline Luz & 80.3 & 77.5 & 87.5 & 83.8 & 59.2 & 76.3 & 74.8 & 72.7 & 49.7 & 75.2 \\
\hline Alcantarillado & 53.5 & 32.0 & 35.4 & 18.8 & 40.1 & 43.8 & 11.7 & 19.8 & 15.6 & 30.0 \\
\hline Telefono & 23.0 & 20.2 & 20.7 & 17.2 & 17.8 & 41.1 & 27.2 & 31.7 & 7.4 & 22.7 \\
\hline \multicolumn{11}{|l|}{ Otros } \\
\hline Transporte & 84.0 & 77.6 & 78.9 & 75.2 & 76.1 & 86.6 & 82.1 & 46.1 & 48.1 & 74.6 \\
\hline Atención Médica & 72.7 & 46.4 & 39.4 & 60.1 & 60.8 & 73.1 & 69.8 & 50.9 & 57.1 & 58.7 \\
\hline Unidades Educativas & 81.3 & 85.5 & 70.5 & 85.6 & 67.6 & 87.9 & 85.8 & 66.9 & 68.6 & 78.9 \\
\hline Colegios & 35.4 & 35.8 & 26.3 & 27.9 & 49.2 & 35.8 & 44.8 & 47.1 & 24.0 & 35.5 \\
\hline Bibliotecas & 15.4 & 7.8 & 8.8 & 9.0 & 17.8 & 25.5 & 19.5 & 25.6 & 6.6 & 14.3 \\
\hline Centros Culturales & 26.0 & 10.5 & 10.3 & 11.8 & 10.4 & 14.5 & 24.5 & 31.7 & 5.7 & 16.1 \\
\hline Areas Recreativas & 17.6 & 38.4 & 32.1 & 34.9 & 26.9 & 46.2 & 44.2 & 49.1 & 25.7 & 34.7 \\
\hline
\end{tabular}


Panel C. Características de la Vivienda

\begin{tabular}{|c|c|c|c|c|c|c|c|c|c|c|}
\hline Agua & 80.9 & 76.9 & 78.0 & 85.6 & 92.2 & 86.6 & 85.8 & 58.0 & 45.1 & 77.7 \\
\hline Luz & 69.1 & 72.7 & 82.1 & 84.0 & 88.0 & 60.5 & 68.2 & 68.6 & 47.0 & 71.7 \\
\hline Alcantarillado & 38.9 & 24.9 & 32.6 & 14.6 & 57.3 & 35.8 & 8.2 & 5.1 & 16.1 & 25.4 \\
\hline Telefono & 2.9 & 6.2 & 16.2 & 4.8 & 8.4 & 18.8 & 7.5 & 6.5 & 6.0 & 8.5 \\
\hline $\begin{array}{l}\text { Más personas que no } \\
\text { estudiaron }\end{array}$ & 39.5 & 38.0 & 33.6 & 32.3 & 38.8 & 30.9 & 34.3 & 37.2 & 36.9 & 35.6 \\
\hline
\end{tabular}

Panel D. Participación PNA-Bolivia

\begin{tabular}{|c|c|c|c|c|c|c|c|c|c|c|}
\hline Entrega Material & & & & & & & & & & \\
\hline Necesita Lentes & 73.8 & 86.0 & 74.3 & 62.7 & 71.8 & 68.3 & 71.4 & 77.8 & 54.1 & 71.7 \\
\hline Entrega de Lentes PNA & 0.8 & 34.0 & 36.9 & 21.0 & 13.9 & 32.8 & 33.0 & 37.5 & 23.0 & 26.0 \\
\hline \multicolumn{11}{|l|}{ Motivaciones* } \\
\hline Aprender a leer y escribir & 91.6 & 72.4 & 82.1 & 84.6 & 86.4 & 73.4 & 62.9 & 82.3 & 75.1 & 78.5 \\
\hline Estudiar erimaria & 7.8 & 9.8 & 11.9 & 19.8 & 11.3 & 5.6 & 4.3 & 7.8 & 4.1 & 9.4 \\
\hline Conseguir el bachillerato & 6.4 & 8.4 & 13.4 & 14.0 & 7.4 & 5.1 & 3.6 & 10.2 & 13.1 & 9.0 \\
\hline económica & 10.0 & 9.3 & 9.5 & 20.0 & 8.1 & 7.5 & 5.5 & 11.3 & 3.6 & 9.6 \\
\hline $\begin{array}{l}\text { Ayudar a la familia en la } \\
\text { escuela }\end{array}$ & 10.7 & 11.8 & 15.5 & 16.8 & 10.0 & 12.4 & 10.3 & 13.7 & 6.3 & 12.1 \\
\hline
\end{tabular}


Tabla 4: Características Socio-Demográficas de los Facilitadores según Departamento

\begin{tabular}{|c|c|c|c|c|c|c|c|c|c|c|}
\hline & Chuquisaca & $\begin{array}{l}\text { La } \\
\text { Paz } \\
\end{array}$ & Cochabamba & Oruro & Potosí & Tarija & $\begin{array}{l}\text { Santa } \\
\text { Cruz }\end{array}$ & Beni & Pando & Bolivia \\
\hline Porcentaje & 15.3 & 13.5 & 13.3 & 14.5 & 9.8 & 8 & 15 & 4.8 & 6 & 100 \\
\hline \multicolumn{11}{|c|}{ Panel A. Características Individuales } \\
\hline \multicolumn{11}{|l|}{ Sexo } \\
\hline Hombre & 36.1 & 44.4 & 34.0 & 31.0 & 46.2 & 31.3 & 30.0 & 21.1 & 20.8 & 34.3 \\
\hline Mujer & 63.9 & 55.6 & 66.0 & 69.0 & 53.8 & 68.8 & 68.3 & 78.9 & 79.2 & 65.5 \\
\hline \multicolumn{11}{|l|}{ Edad } \\
\hline 15 a 25 & 21.3 & 24.1 & 20.8 & 17.2 & 7.7 & 31.3 & 16.7 & 10.5 & 41.7 & 20.5 \\
\hline 26 a 35 & 49.2 & 48.1 & 34.0 & 43.1 & 28.2 & 25.0 & 41.7 & 36.8 & 29.2 & 39.3 \\
\hline 36 a 45 & 18.0 & 16.7 & 32.1 & 32.8 & 43.6 & 34.4 & 21.7 & 21.1 & 16.7 & 26.3 \\
\hline 46 a 55 & 8.2 & 9.3 & 11.3 & 3.4 & 15.4 & 9.4 & 16.7 & 31.6 & 4.2 & 11.0 \\
\hline 56 a 65 & 0.0 & 0.0 & 1.9 & 0.0 & 2.6 & 0.0 & 1.7 & 0.0 & 0.0 & 0.8 \\
\hline 66 a 75 & 0.0 & 1.9 & 0.0 & 0.0 & 0.0 & 0.0 & 0.0 & 0.0 & 4.2 & 0.5 \\
\hline $\mathrm{NS} / \mathrm{NR}$ & 3.3 & 0.0 & 0.0 & 3.4 & 2.6 & 0.0 & 1.7 & 0.0 & 4.2 & 1.8 \\
\hline \multicolumn{11}{|l|}{ Idioma } \\
\hline Quechua & 19.7 & 0.0 & 3.8 & 5.2 & 2.6 & 0.0 & 0.0 & 0.0 & 0.0 & 4.5 \\
\hline Aymara & 0.0 & 0.0 & 3.8 & 0.0 & 0.0 & 0.0 & 0.0 & 0.0 & 0.0 & 0.5 \\
\hline Castellano & 77.0 & 98.1 & 92.5 & 87.9 & 97.4 & 96.9 & 91.7 & 94.7 & 91.7 & 91.0 \\
\hline Extranjero & 3.3 & 1.9 & 0.0 & 5.2 & 0.0 & 3.1 & 0.0 & 5.3 & 8.3 & 2.5 \\
\hline Otro & 0.0 & 0.0 & 0.0 & 1.7 & 0.0 & 0.0 & 8.3 & 0.0 & 0.0 & 1.5 \\
\hline \multicolumn{11}{|l|}{ Raza* } \\
\hline Amarilla & 0.0 & 0.0 & 0.0 & 0.0 & 100.0 & 0.0 & 0.0 & 0.0 & 0.0 & 100.0 \\
\hline Blanca & 12.1 & 10.6 & 13.6 & 9.1 & 4.5 & 1.5 & 31.8 & 6.1 & 10.6 & 100.0 \\
\hline Indígena & 30.6 & 38.7 & 4.8 & 3.2 & 4.8 & 1.6 & 12.9 & 0.0 & 3.2 & 100.0 \\
\hline Mestiza & 13.3 & 8.2 & 15.6 & 18.8 & 11.3 & 11.7 & 11.7 & 3.5 & 5.9 & 100.0 \\
\hline Negra & 0.0 & 100.0 & 0.0 & 0.0 & 0.0 & 0.0 & 0.0 & 0.0 & 0.0 & 100.0 \\
\hline NS/NR & 0.0 & 0.0 & 1.9 & 3.4 & 7.7 & 0.0 & 1.7 & 31.6 & 0.0 & 3.3 \\
\hline \multicolumn{11}{|c|}{ Educación Superior } \\
\hline Licenciatura & 9.8 & 14.8 & 15.1 & 17.2 & 28.2 & 9.4 & 10.0 & 10.5 & 8.3 & 14.0 \\
\hline Normal & 44.3 & 48.1 & 75.5 & 56.9 & 74.4 & 9.4 & 53.3 & 42.1 & 12.5 & 50.2 \\
\hline Otro & 45.9 & 37.1 & 9.4 & 25.9 & 0.0 & 81.2 & 36.7 & 47.4 & 79.2 & 35.8 \\
\hline \multicolumn{11}{|c|}{ Grupo Étnico* } \\
\hline Quechua & 93.4 & 11.1 & 88.7 & 55.2 & 100.0 & 21.9 & 28.3 & 0.0 & 12.5 & 52.0 \\
\hline Aymara & 1.6 & 83.3 & 7.5 & 37.9 & 0.0 & 3.1 & 1.7 & 0.0 & 16.7 & 19.5 \\
\hline Ninguno & 4.9 & 1.9 & 3.8 & 6.9 & 0.0 & 68.8 & 28.3 & 21.1 & 66.7 & 17.3 \\
\hline Chiquitano & 0.0 & 0.0 & 0.0 & 0.0 & 0.0 & 0.0 & 28.3 & 0.0 & 0.0 & 4.3 \\
\hline Guarani & 0.0 & 0.0 & 0.0 & 0.0 & 0.0 & 0.0 & 8.3 & 0.0 & 0.0 & 1.3 \\
\hline Otros & 0.0 & 3.7 & 0.0 & 0.0 & 0.0 & 3.1 & 1.7 & 68.4 & 4.2 & 4.5 \\
\hline $\mathrm{NS} / \mathrm{NR}$ & 0.0 & 0.0 & 0.0 & 0.0 & 0.0 & 3.1 & 3.3 & 10.5 & 0.0 & 1.3 \\
\hline
\end{tabular}

Panel A. Programa PNA Bolivia

Suficiencia en conocimientos** de:

\begin{tabular}{|c|c|c|c|c|c|c|c|c|c|}
\hline Zona donde trabaja & 88.5 & 77.8 & 71.7 & 87.9 & 82.1 & 87.5 & 86.7 & 68.4 & 75.0 \\
\hline Participantes & 75.4 & 72.2 & 66.0 & 58.6 & 79.5 & 71.9 & 83.3 & 78.9 & 95.8 \\
\hline $\begin{array}{l}\text { Elaboración de Diagnósticos a } \\
\text { participantes }\end{array}$ & 78.7 & 81.5 & 69.8 & 51.7 & 61.5 & 65.6 & 83.3 & 78.9 & 91.7 \\
\hline Pedagogía del adulto & 47.5 & 53.7 & 37.7 & 36.2 & 43.6 & 31.3 & 51.7 & 57.9 & 8.3 \\
\hline
\end{tabular}


Metodología de enseñanza de lengua

\begin{tabular}{|c|c|c|c|c|c|c|c|c|c|}
\hline 65.6 & 72.2 & 64.2 & 56.9 & 69.2 & 68.8 & 71.7 & 63.2 & 83.3 & 67.5 \\
\hline 85.2 & 74.1 & 56.6 & 74.1 & 71.8 & 90.6 & 98.3 & 89.5 & 100.0 & 80.5 \\
\hline 83.6 & 63.0 & 60.4 & 69.0 & 43.6 & 75.0 & 83.3 & 73.7 & 87.5 & 70.8 \\
\hline 68.9 & 72.2 & 47.2 & 44.8 & 64.1 & 9.4 & 35.0 & 21.1 & 12.5 & 47.0 \\
\hline 4.6 & 37.0 & 35.8 & 55.2 & 41.0 & 25.0 & 35.0 & 52.6 & 20.8 & 36.5 \\
\hline 59.0 & 50.0 & 34.0 & 43.1 & 59.0 & 31.3 & 35.0 & 52.6 & 25.0 & 44.0 \\
\hline 6.6 & 11.1 & 9.4 & 5.2 & 5.1 & 6.3 & 1.7 & 5.3 & 0.0 & 6.0 \\
\hline 1.1 & 55.6 & 52.8 & 46.6 & 48.7 & 62.5 & 38.3 & 73.7 & 45.8 & 47.8 \\
\hline 1.6 & 11.1 & 15.1 & 15.5 & 5.1 & 6.3 & 5.0 & 15.8 & 0.0 & 8.5 \\
\hline 6.6 & 3.7 & 0.0 & 5.2 & 0.0 & 0.0 & 1.7 & 5.3 & 8.3 & 3.3 \\
\hline 82.0 & 87.0 & 92.5 & 91.4 & 97.4 & 90.6 & 78.3 & 89.5 & 87.5 & 87.8 \\
\hline 3.3 & 16.7 & 15.1 & 27.6 & 10.3 & 12.5 & 1.7 & 26.3 & 0.0 & 12.3 \\
\hline 11.5 & 29.6 & 24.5 & 27.6 & 10.3 & 9.4 & 8.3 & 42.1 & 4.2 & 18.3 \\
\hline 3.3 & 16.7 & 11.3 & 20.7 & 5.1 & 3.1 & 3.3 & 21.1 & 0.0 & 9.5 \\
\hline 14.8 & 29.6 & 32.1 & 34.5 & 28.2 & 28.1 & 16.7 & 57.9 & 4.2 & 26.0 \\
\hline 4.9 & 16.7 & 13.2 & 17.2 & 10.3 & 6.3 & 5.0 & 31.6 & 0.0 & 11.0 \\
\hline 4.9 & 20.4 & 13.2 & 22.4 & 12.8 & 12.5 & 5.0 & 36.8 & 0.0 & 13.3 \\
\hline 18.0 & 25.9 & 24.5 & 24.1 & 20.5 & 15.6 & 11.7 & 42.1 & 4.2 & 20.3 \\
\hline 0.0 & 0.0 & 1.9 & 1.7 & 0.0 & 0.0 & 1.7 & 5.3 & 0.0 & 1.0 \\
\hline 95.1 & 96.3 & 98.1 & 87.9 & 100.0 & 96.9 & 100.0 & 100.0 & 100.0 & 96.5 \\
\hline 20 & 70.4 & 84.9 & 75.9 & 92.3 & 75.0 & 93.3 & 78.9 & 83.3 & 82.0 \\
\hline
\end{tabular}

Método "Yo Si Puedo"

Evaluación Final

Educación intercultural bilingue

Motivo de Participación en “Yo S

Puedo"

Aprender más como docente

Compromiso con analfabetos

Mejorar condiciones económicas

Disminuir analfabetismo

Puntos para el escalafon

Otros

Motivación de Participantes

Aprender a leer y escribir

Ser mejor trabajador

Ser mejor ciudadano

Mejorar condición económica

Ayudar a hijos en la escuela

Buscar mejor trabajo

Continuar estudios superiores

No ser marginado

Otro

Satisfacción con ser facilitador

Satisfecho

Ocupado fuera del Programa "Yo Si

$\begin{array}{rrrr}84.9 & 75.9 & 92.3 & 75.0\end{array}$

Fuente: Elaboración de los autores en base a la Encuesta de Diagnóstico Socio-Cultural de los Participantes del PNA, Ministerio de Educación y Culturas.

*Autodeclarado por el facilitador. El encuestado puede dar más de una respuesta

**Personas que consideran tener suficiente conocimiento en la lista de características descritas. El encuestado puede dar más de una respuesta 
Tabla 5: Características Socio-Demográficas según Participación en el PNA-Yo Si Puedo. Chuquisaca

\begin{tabular}{|c|c|c|c|c|c|c|}
\hline & \multicolumn{3}{|c|}{ Participantes PNA-Yo Si Puedo } & \multicolumn{3}{|c|}{ No Participantes PNA-Yo Si Puedo } \\
\hline & Urbano & Rural & $\begin{array}{c}\text { Total } \\
\text { Chuquisaca }\end{array}$ & Urbano & Rural & $\begin{array}{c}\text { Total } \\
\text { Chuquisaca }\end{array}$ \\
\hline \multicolumn{7}{|c|}{ Características Individuales ( \%) } \\
\hline \multicolumn{7}{|l|}{ Sexo } \\
\hline Hombre & 8.3 & 20.6 & 15.4 & 26.3 & 43.6 & 35.2 \\
\hline Mujer & 91.7 & 79.4 & 84.6 & 73.7 & 56.4 & 64.8 \\
\hline \multicolumn{7}{|l|}{ Edad } \\
\hline 15 a 25 & 10.0 & 5.0 & 7.1 & 15.9 & 14.6 & 15.3 \\
\hline 26 a 35 & 25.8 & 15.0 & 19.6 & 26.0 & 18.9 & 22.3 \\
\hline 36 a 45 & 28.3 & 27.5 & 27.9 & 26.9 & 23.8 & 25.3 \\
\hline 46 a 55 & 19.2 & 22.5 & 21.1 & 19.5 & 19.8 & 19.7 \\
\hline 56 a 65 & 16.7 & 30.0 & 24.3 & 11.7 & 22.9 & 17.5 \\
\hline \multicolumn{7}{|l|}{ Educación } \\
\hline Ninguno & 21.7 & 28.7 & 25.7 & 16.6 & 24.4 & 20.6 \\
\hline 1ro Básico & 14.2 & 12.5 & 13.2 & 10.7 & 6.7 & 8.6 \\
\hline 2do Básico & 19.2 & 15.0 & 16.8 & 12.0 & 11.3 & 11.6 \\
\hline 3ro Básico & 15.0 & 13.1 & 13.9 & 17.9 & 13.4 & 15.6 \\
\hline 4to Básico & 8.3 & 10.0 & 9.3 & 14.6 & 12.8 & 13.7 \\
\hline 5to Básico & 21.7 & 20.6 & 21.1 & 28.2 & 31.4 & 29.9 \\
\hline \multicolumn{7}{|l|}{ Idioma } \\
\hline Castellano & 76.7 & 85.6 & 81.8 & 82.1 & 79.0 & 80.5 \\
\hline Quechua & 23.3 & 14.4 & 18.2 & 17.9 & 20.4 & 19.2 \\
\hline Aymara & 0.0 & 0.0 & 0.0 & 0.0 & 0.3 & 0.2 \\
\hline Guaraní & 0.0 & 0.0 & 0.0 & 0.0 & 0.3 & 0.2 \\
\hline \multicolumn{7}{|l|}{ Estado Civil } \\
\hline Soltero(a) & 15.0 & 15.0 & 15.0 & 11.7 & 25.3 & 18.7 \\
\hline Casado(a) & 58.3 & 66.9 & 63.2 & 67.2 & 49.4 & 58.0 \\
\hline Concubinato(a) & 19.2 & 12.5 & 15.4 & 16.2 & 14.6 & 15.4 \\
\hline Separado(a) & 2.5 & 0.0 & 1.1 & 0.6 & 1.2 & 0.9 \\
\hline Divorciado(a) & 0.0 & 0.0 & 0.0 & 0.3 & 0.3 & 0.3 \\
\hline Viudo(a) & 4.2 & 5.6 & 5.0 & 3.9 & 8.5 & 6.3 \\
\hline $\mathrm{NS} / \mathrm{NR}$ & 0.8 & 0.0 & 0.4 & 0.0 & 0.3 & 0.2 \\
\hline \multicolumn{7}{|l|}{ Hijos } \\
\hline No & 4.2 & 7.5 & 6.1 & 8.8 & 12.5 & 10.7 \\
\hline $\mathrm{Si}$ & 95.8 & 92.5 & 93.9 & 91.2 & 87.2 & 89.2 \\
\hline \multicolumn{7}{|c|}{ Educación Padre } \\
\hline Primaria & 18.3 & 25.6 & 22.5 & 31.8 & 29.9 & 30.8 \\
\hline Secundaria & 5.8 & 0.0 & 2.5 & 1.6 & 0.3 & 0.9 \\
\hline
\end{tabular}




\begin{tabular}{|c|c|c|c|c|c|c|}
\hline Superior & 0.0 & 0.6 & 0.4 & 0.3 & 0.9 & 0.6 \\
\hline No fue a la escuela & 61.7 & 63.1 & 62.5 & 54.2 & 58.2 & 56.3 \\
\hline No conoció a su padre & 10.8 & 9.4 & 10.0 & 8.4 & 9.5 & 9.0 \\
\hline $\mathrm{NS} / \mathrm{NR}$ & 3.3 & 1.3 & 2.1 & 3.6 & 1.2 & 2.4 \\
\hline \multicolumn{7}{|l|}{ Educación Madre } \\
\hline Primaria & 16.7 & 15.6 & 16.1 & 19.8 & 16.8 & 18.2 \\
\hline Superior & 0.0 & 1.3 & 0.7 & 0.3 & 0.0 & 0.2 \\
\hline No fue la escuela & 75.8 & 73.8 & 74.6 & 72.1 & 76.5 & 74.4 \\
\hline No conocí a mi madre & 5.0 & 8.1 & 6.8 & 3.9 & 5.8 & 4.9 \\
\hline $\mathrm{NS} / \mathrm{NR}$ & 2.5 & 1.3 & 1.8 & 3.9 & 0.9 & 2.4 \\
\hline \multicolumn{7}{|c|}{ Ocupación } \\
\hline \multicolumn{7}{|l|}{ Horas Trabajadas (Hrs.) } \\
\hline Media & 9.8 & 11.6 & 10.5 & 10.9 & 11.2 & 11.1 \\
\hline Desv. Estándar & 6.0 & 3.8 & 4.9 & 5.7 & 4.7 & 5.2 \\
\hline \multicolumn{7}{|l|}{ Dias Trabajados (Días) } \\
\hline Media & 6.5 & 6.6 & 6.5 & 6.4 & 6.5 & 6.4 \\
\hline Desv. Estándar & 0.8 & 1.0 & 0.9 & 1.0 & 0.9 & 0.9 \\
\hline \multicolumn{7}{|c|}{ Meses Trabajados en un año (Meses) } \\
\hline Media & 11.3 & 11.1 & 11.2 & 11.2 & 11.3 & 11.2 \\
\hline Desv. Estándar & 2.4 & 2.0 & 2.2 & 2.2 & 1.9 & 2.1 \\
\hline \multicolumn{7}{|l|}{ Lee Direcciones } \\
\hline Todos los días & 7.5 & 5.6 & 6.4 & 10.7 & 3.0 & 6.8 \\
\hline Varios días a la semana & 7.5 & 2.5 & 4.6 & 4.9 & 3.7 & 4.2 \\
\hline Una vez a la semana & 5.8 & 3.1 & 4.3 & 4.5 & 3.7 & 4.1 \\
\hline Algunas veces al mes & 20.0 & 20.0 & 20.0 & 19.5 & 19.5 & 19.5 \\
\hline Nunca & 51.7 & 54.4 & 53.2 & 53.9 & 61.0 & 57.5 \\
\hline NS/NR & 7.5 & 14.4 & 11.4 & 6.5 & 9.1 & 7.9 \\
\hline \multicolumn{7}{|c|}{ Realiza Calc. Matemáticos } \\
\hline Todos los días & 26.7 & 7.5 & 15.7 & 24.7 & 7.9 & 16.0 \\
\hline Varios días a la semana & 8.3 & 6.3 & 7.1 & 7.1 & 7.6 & 7.4 \\
\hline Una vez a la semana & 5.8 & 6.3 & 6.1 & 4.5 & 6.1 & 5.3 \\
\hline Algunas veces al mes & 19.2 & 31.3 & 26.1 & 24.0 & 28.0 & 26.1 \\
\hline Nunca & 34.2 & 34.4 & 34.3 & 34.1 & 41.8 & 38.1 \\
\hline NS/NR & 5.8 & 14.4 & 10.7 & 5.5 & 8.5 & 7.1 \\
\hline \multicolumn{7}{|l|}{ Escribe Notas } \\
\hline Todos los días & 5.8 & 3.8 & 4.6 & 6.5 & 4.0 & 5.2 \\
\hline Varios días a la semana & 4.2 & 6.3 & 5.4 & 5.5 & 3.7 & 4.6 \\
\hline Una vez a la semana & 8.3 & 3.8 & 5.7 & 5.5 & 3.7 & 4.6 \\
\hline Algunas veces al mes & 15.0 & 18.1 & 16.8 & 16.2 & 19.2 & 17.8 \\
\hline Nunca & 60.0 & 55.0 & 57.1 & 61.0 & 61.3 & 61.2 \\
\hline NS/NR & 6.7 & 13.1 & 10.4 & 5.2 & 8.2 & 6.8 \\
\hline
\end{tabular}

Fuente: Elaboración de los autores en base a la Encuesta "Yo Si Puedo" Chuquisaca realizada por la Red Boliviana de Microdatos y Encuestas 
Tabla 6: Percepción de Participantes del PNA-Yo Si Puedo. Chuquisaca

\begin{tabular}{|c|c|c|c|}
\hline & Urbano & Rural & $\begin{array}{c}\text { Total } \\
\text { Chuquisaca }\end{array}$ \\
\hline \multicolumn{4}{|c|}{ PNA - Yo Si Puedo } \\
\hline \multicolumn{4}{|l|}{ Razones de Ingreso a PNA } \\
\hline Aprender a leer y escribir & 71.7 & 86.9 & 80.4 \\
\hline Estudiar primaria y continuar el bachillerato & 1.7 & 3.1 & 2.5 \\
\hline Conseguir trabajo & 1.7 & 0.0 & 0.7 \\
\hline Mejorar situación económica & 5.0 & 1.3 & 2.9 \\
\hline Otro & 6.7 & 5.0 & 5.7 \\
\hline NS/NR & 13.3 & 3.8 & 7.9 \\
\hline \multicolumn{4}{|l|}{ Idioma que fue Alfabetizado } \\
\hline Castellano & 40.0 & 64.4 & 53.9 \\
\hline Quechua & 21.7 & 8.1 & 13.9 \\
\hline NS/NR & 2.5 & 2.5 & 2.5 \\
\hline Castellano y quechua & 35.8 & 25.0 & 29.6 \\
\hline \multicolumn{4}{|l|}{ Opinión de Materiales } \\
\hline Buena & 61.7 & 58.1 & 59.6 \\
\hline Regular & 30.8 & 31.3 & 31.1 \\
\hline Deficiente (mala) & 5.0 & 4.4 & 4.6 \\
\hline $\mathrm{NS} / \mathrm{NR}$ & 2.5 & 6.3 & 4.6 \\
\hline \multicolumn{4}{|l|}{ Opinión de Método } \\
\hline Buena & 65.0 & 60.6 & 62.5 \\
\hline Regular & 25.8 & 33.1 & 30.0 \\
\hline Deficiente (mala) & 6.7 & 4.4 & 5.4 \\
\hline NS/NR & 2.5 & 1.9 & 2.1 \\
\hline \multicolumn{4}{|l|}{ Opinión de Facilitadores } \\
\hline Buena & 65.8 & 61.9 & 63.6 \\
\hline Regular & 21.7 & 33.8 & 28.6 \\
\hline Deficiente (mala) & 5.8 & 2.5 & 3.9 \\
\hline $\mathrm{NS} / \mathrm{NR}$ & 6.7 & 1.9 & 3.9 \\
\hline \multicolumn{4}{|l|}{ Recibió Lentes } \\
\hline $\mathrm{Si}$ & 8.3 & 18.8 & 14.3 \\
\hline No & 87.5 & 80.6 & 83.6 \\
\hline $\mathrm{NS} / \mathrm{NR}$ & 4.2 & 0.0 & 1.8 \\
\hline \multicolumn{4}{|l|}{ Recibieron Panel Solar } \\
\hline $\mathrm{Si}$ & 3.3 & 20.6 & 13.2 \\
\hline No & 95.8 & 74.4 & 83.6 \\
\hline $\mathrm{NS} / \mathrm{NR}$ & 0.8 & 5.0 & 3.2 \\
\hline \multicolumn{4}{|l|}{ Utilizaron Video } \\
\hline $\mathrm{Si}$ & 49.2 & 73.1 & 62.9 \\
\hline No & 50.8 & 26.9 & 37.1 \\
\hline \multicolumn{4}{|l|}{ Recibió Libreta de Aprobación } \\
\hline $\mathrm{Si}$ & 42.5 & 50.0 & 46.8 \\
\hline No & 57.5 & 49.4 & 52.9 \\
\hline $\mathrm{NS} / \mathrm{NR}$ & 0.0 & 0.6 & 0.4 \\
\hline \multicolumn{4}{|l|}{ Asiste Programa Post-Alfabetización } \\
\hline $\mathrm{Si}$ & 50.8 & 51.9 & 51.4 \\
\hline No & 46.7 & 46.9 & 46.8 \\
\hline $\mathrm{NS} / \mathrm{NR}$ & 2.5 & 1.3 & 1.8 \\
\hline
\end{tabular}

Fuente: Elaboración de los autores en base a la Encuesta "Yo Si Puedo" Chuquisaca realizada por la Red Boliviana de Microdatos y Encuestas 
Tabla 7: Tasas de Analfabetismo, Variación Anual Intercensal y Esfuerzo Necesario para Erradicación

\begin{tabular}{|c|c|c|c|c|c|c|c|c|}
\hline & \multicolumn{3}{|c|}{ Tasas de Analfabetismo } & \multicolumn{2}{|c|}{ Variación Anual Intercensal } & \multicolumn{3}{|c|}{$\begin{array}{c}\text { Esfuerzo para erradicar el } \\
\text { analfabetismo }<4 \%\end{array}$} \\
\hline & (1) & (2) & (3) & (4) & (5) & (6) & (7) & (8) \\
\hline & 1976 & 1992 & 2001 & 1976-1992 & 1992-2001 & $\begin{array}{l}\text { Proyec } \\
2010^{*}\end{array}$ & $\begin{array}{l}\text { T. } \\
\text { Óptima*** }\end{array}$ & Esfuerzo $* * *$ \\
\hline Bolivia & 36.79 & 20 & 13.3 & -3.74 & -4.43 & 8.8 & -10.35 & 2.33 \\
\hline \multicolumn{9}{|c|}{ Panel A. Departamentos } \\
\hline Chuquisaca & 61.15 & 39.48 & 28.36 & -2.7 & -3.61 & 20.4 & -16.31 & 4.52 \\
\hline $\mathrm{La} \mathrm{Paz}$ & 31.93 & 16.87 & 11.92 & -3.91 & -3.79 & 8.4 & -9.45 & 2.5 \\
\hline Cochabamba & 40.79 & 21.19 & 14.32 & -4.01 & -4.26 & 9.7 & -10.95 & 2.57 \\
\hline Oruro & 26.88 & 15.39 & 11.26 & -3.43 & -3.41 & 8.2 & -8.98 & 2.63 \\
\hline Potosi & 54.47 & 38.19 & 30.06 & -2.19 & -2.62 & 23.7 & -16.75 & 6.38 \\
\hline Tarija & 36.91 & 21.22 & 15.39 & -3.4 & -3.51 & 11.2 & -11.53 & 3.29 \\
\hline Santa Cruz & 21.08 & 11.07 & 7.64 & -3.95 & -4.04 & 5.3 & -5.71 & 1.42 \\
\hline Beni & 22.01 & 12.84 & 9.59 & -3.31 & -3.19 & 7.2 & -7.64 & 2.39 \\
\hline Pando & 33.58 & 21.02 & 15.53 & -2.89 & -3.31 & 11.5 & -11.6 & 3.51 \\
\hline \multicolumn{9}{|c|}{ Panel B. Grupos etáreos } \\
\hline $15-24$ & 16.45 & 6.1 & 4.2 & -6.01 & -4.06 & 2.9 & -0.44 & 0.11 \\
\hline $25-34$ & 29.87 & 11.23 & 8.13 & -5.93 & -3.53 & 5.9 & -6.24 & 1.77 \\
\hline $35-44$ & 45.05 & 28.08 & 20.16 & -2.91 & -3.61 & 14.5 & -13.67 & 3.78 \\
\hline $45-54$ & 54.25 & 33.79 & 24.11 & -2.92 & -3.68 & 17.2 & -15.07 & 4.09 \\
\hline $55-64$ & 64.56 & 45.4 & 32.2 & -2.18 & -3.75 & 22.8 & -17.27 & 4.61 \\
\hline$>65$ & 74.48 & 59.59 & 40.21 & -1.38 & -4.28 & 27.1 & -18.93 & 4.43 \\
\hline
\end{tabular}

Fuente: Elaboración de los autores en base a datos del INE, Censo Nacional de Población y Vivienda de Bolivia 1976, 1992 y 2001

Nota: Las tasas de variación intercensal del periodo 1976-1992 y 1992-2001 fueron obtenidas de la siguiente forma:

\$lleft[ $\backslash$ left( $\left.\left(\mid \operatorname{frac}\left\{\mathrm{r}_{-}\left\{\mathrm{t} \_\{2\}\right\}\right\}\left\{\mathrm{r}_{-}\left\{\mathrm{t} \_\{1\}\right\}\right\} \backslash \text { right }\right)^{\wedge}\left\{1 /\left(\mathrm{t} \_\{2\}-\mathrm{t} \_\{1\}\right)\right\} \backslash \operatorname{right}\right]-1 \$$

* Es la proyección al año 2010 con la tasa de variación intercensal 1992-2001

** Es la tasa anual de reducción del analfabetismo necesaria para tener una tasa de analfabetismo menor a $4\lfloor \%$ en 2010

*** El esfuerzo es el ratio de la Tasa óptima (7) entre la Tasa de variación intercensal 1992-2001 (5) 
Tabla 8: Tasa de Analfabetismo Según Año de Nacimiento por Área, Sexo y Departamento

\begin{tabular}{|c|c|c|c|c|c|c|c|c|c|c|c|c|c|c|}
\hline & 1920 & 1925 & 1930 & 1935 & 1940 & 1945 & 1950 & 1955 & 1960 & 1965 & 1970 & 1975 & 1980 & 1985 \\
\hline Bolivia & 60.5 & 53.8 & 49.0 & 44.5 & 40.5 & 31.2 & 25.3 & 18.1 & 14.0 & 10.6 & 7.5 & 5.2 & 3.6 & 1.8 \\
\hline Urbano & 45.2 & 34.1 & 30.2 & 27.9 & 22.1 & 17.5 & 12.9 & 8.7 & 6.4 & 5.0 & 3.1 & 2.5 & 1.5 & 0.7 \\
\hline Hombre & 29.4 & 16.2 & 10.7 & 10.6 & 8.0 & 6.5 & 3.5 & 2.9 & 1.7 & 1.2 & 1.2 & 0.9 & 0.8 & 0.5 \\
\hline Mujer & 54.6 & 46.7 & 46.6 & 42.3 & 34.3 & 27.2 & 21.2 & 13.9 & 10.8 & 8.2 & 4.9 & 4.0 & 2.2 & 0.9 \\
\hline Chuquisaca & 53.3 & 41.9 & 35.8 & 37.5 & 30.4 & 25.6 & 25.8 & 14.3 & 11.9 & 9.7 & 6.6 & 7.2 & 2.8 & 1.2 \\
\hline La Paz & 46.3 & 33.3 & 33.9 & 29.3 & 23.0 & 18.1 & 11.7 & 9.0 & 5.2 & 5.8 & 2.6 & 1.9 & 1.0 & 0.6 \\
\hline Cochabamba & 36.0 & 29.9 & 23.4 & 23.4 & 19.1 & 15.4 & 11.1 & 6.7 & 7.4 & 5.4 & 3.4 & 2.8 & 1.8 & 0.7 \\
\hline Oruro & 70.3 & 49.0 & 38.9 & 29.0 & 23.6 & 20.8 & 10.5 & 7.0 & 6.6 & 3.8 & 2.3 & 0.5 & 0.5 & 0.5 \\
\hline Potosi & 66.9 & 43.0 & 39.0 & 40.0 & 37.6 & 28.5 & 23.3 & 13.6 & 9.3 & 7.4 & 7.1 & 4.2 & 1.9 & 0.8 \\
\hline Tarija & 51.8 & 36.8 & 28.1 & 30.8 & 28.9 & 23.0 & 17.5 & 14.4 & 6.5 & 4.7 & 3.4 & 2.2 & 1.8 & 1.3 \\
\hline Santa Cruz & 41.1 & 27.1 & 25.7 & 22.5 & 15.1 & 12.0 & 9.9 & 7.5 & 5.8 & 2.8 & 2.2 & 2.5 & 1.9 & 0.7 \\
\hline Beni & 30.2 & 43.2 & 26.7 & 26.1 & 17.7 & 13.4 & 7.5 & 5.5 & 6.0 & 4.5 & 2.0 & 1.1 & 1.0 & 0.7 \\
\hline Pando & 0.0 & 19.0 & 0.0 & 6.2 & 24.7 & 6.5 & 6.6 & 0.9 & 3.0 & 2.1 & 2.2 & 2.0 & 1.9 & 0.0 \\
\hline Rural & 78.6 & 73.5 & 69.5 & 61.0 & 59.0 & 47.8 & 43.9 & 33.8 & 28.1 & 21.4 & 16.9 & 11.6 & 9.4 & 4.5 \\
\hline Hombre & 58.5 & 51.4 & 49.1 & 36.4 & 37.4 & 23.8 & 21.6 & 15.8 & 12.9 & 10.3 & 7.0 & 4.9 & 3.9 & 2.1 \\
\hline Mujer & 94.4 & 92.0 & 88.9 & 83.0 & 80.5 & 69.8 & 65.1 & 51.9 & 43.3 & 32.6 & 26.1 & 17.9 & 14.5 & 7.0 \\
\hline Chuquisaca & 83.1 & 78.3 & 82.5 & 80.6 & 73.8 & 67.4 & 60.1 & 52.1 & 45.0 & 35.7 & 30.1 & 14.0 & 13.6 & 4.7 \\
\hline $\mathrm{La} \mathrm{Paz}$ & 76.0 & 64.3 & 68.2 & 51.8 & 56.0 & 43.5 & 34.7 & 25.4 & 17.9 & 10.6 & 9.3 & 5.2 & 4.8 & 2.7 \\
\hline Cochabamba & 82.5 & 85.8 & 73.8 & 78.4 & 66.3 & 51.1 & 46.6 & 35.3 & 34.7 & 29.1 & 21.7 & 17.3 & 13.2 & 5.3 \\
\hline Oruro & 86.0 & 65.7 & 56.3 & 46.7 & 42.4 & 31.1 & 29.2 & 14.3 & 10.2 & 5.6 & 3.0 & 4.0 & 1.5 & 2.6 \\
\hline Potosi & 90.6 & 88.7 & 79.8 & 71.1 & 67.0 & 55.5 & 55.5 & 42.5 & 36.5 & 28.1 & 23.8 & 18.2 & 11.4 & 7.7 \\
\hline Tarija & 60.9 & 84.6 & 62.5 & 62.1 & 55.3 & 51.8 & 48.1 & 45.6 & 25.1 & 19.1 & 16.3 & 11.2 & 11.4 & 7.2 \\
\hline Santa Cruz & 52.4 & 49.9 & 65.3 & 49.5 & 49.5 & 41.2 & 43.7 & 32.7 & 25.7 & 21.0 & 11.6 & 8.1 & 8.1 & 3.1 \\
\hline Beni & 72.5 & 62.8 & 53.0 & 41.9 & 33.3 & 18.5 & 33.0 & 21.2 & 12.5 & 10.5 & 11.7 & 8.3 & 7.1 & 3.5 \\
\hline Pando & 50.5 & 68.2 & 28.9 & 33.4 & 42.9 & 29.1 & 18.3 & 28.4 & 12.5 & 12.7 & 4.7 & 8.5 & 6.7 & 2.9 \\
\hline
\end{tabular}

Fuente: Elaboración de los autores en base a la serie armonizada de Encuesta de Hogares de la Fundación ARU 
Tabla 9: Evolución de las Tasas de Analfabetismo y Población Analfabeta por Área y Sexo

\begin{tabular}{|c|c|c|c|c|c|c|c|c|c|c|c|}
\hline & 1999 & 2000 & 2001 & 2002 & 2003 & 2004 & 2005 & 2006 & 2007 & 2008 & 2009 \\
\hline \multicolumn{12}{|c|}{ Panel A: Muestra: Mayores a 15 años } \\
\hline Bolivia & 15.2 & 14.2 & 13.9 & 12.7 & 13.0 & 12.7 & 11.2 & 10.4 & 10.0 & 9.4 & 9.0 \\
\hline Urbano & 5.3 & 6.2 & 7.3 & 5.9 & 6.4 & 6.2 & 5.4 & 4.8 & 4.2 & 4.1 & 4.7 \\
\hline Hombre & 1.7 & 2.5 & 3.0 & 1.7 & 2.3 & 2.2 & 1.8 & 1.9 & 1.1 & 1.5 & 1.9 \\
\hline Mujer & 8.5 & 9.5 & 11.1 & 9.5 & 9.9 & 9.6 & 8.6 & 7.5 & 7.0 & 6.4 & 7.4 \\
\hline Rural & 33.7 & 29.0 & 26.3 & 25.7 & 26.0 & 25.7 & 23.3 & 21.9 & 22.2 & 20.6 & 18.4 \\
\hline Hombre & 18.6 & 16.6 & 12.9 & 13.7 & 13.7 & 14.3 & 11.4 & 10.9 & 11.1 & 11.5 & 9.1 \\
\hline Mujer & 48.3 & 41.1 & 39.8 & 37.7 & 38.5 & 36.9 & 34.8 & 32.3 & 32.7 & 29.1 & 27.2 \\
\hline \multicolumn{12}{|c|}{ Panel B: Muestra: Mayores a 25 años } \\
\hline Bolivia & 21.0 & 19.5 & 19.3 & 17.9 & 18.2 & 17.8 & 15.3 & 14.2 & 14.0 & 13.0 & 12.4 \\
\hline Urbano & 7.7 & 8.9 & 10.3 & 8.5 & 9.3 & 9.0 & 7.7 & 6.8 & 5.8 & 5.9 & 6.7 \\
\hline Hombre & 2.3 & 3.4 & 4.0 & 2.3 & 3.3 & 3.1 & 2.4 & 2.6 & 1.5 & 2.0 & 2.6 \\
\hline Mujer & 12.5 & 13.8 & 15.9 & 14.0 & 14.4 & 14.0 & 12.2 & 10.6 & 9.6 & 9.3 & 10.4 \\
\hline Rural & 42.6 & 37.3 & 34.8 & 34.5 & 33.7 & 33.6 & 30.0 & 28.1 & 29.9 & 26.8 & 23.8 \\
\hline Hombre & 23.8 & 21.7 & 17.1 & 18.8 & 17.8 & 18.7 & 14.7 & 14.7 & 15.2 & 15.1 & 11.6 \\
\hline Mujer & 60.2 & 52.5 & 51.9 & 49.8 & 49.9 & 47.8 & 44.3 & 40.5 & 43.3 & 37.6 & 35.4 \\
\hline
\end{tabular}

Fuente: Elaboración de los autores en base a la serie armonizada de Encuesta de Hogares de la Fundación ARU

Tabla 10: Evolución de la Variación de las Tasas de Analfabetismo y Población Analfabeta por Muestra, Área y Sexo

\begin{tabular}{|c|c|c|c|c|c|c|c|c|c|c|c|}
\hline & & 2000 & 2001 & 2002 & 2003 & 2004 & 2005 & 2006 & 2007 & 2008 & 2009 \\
\hline \multicolumn{12}{|c|}{ Panel A: Tasa de Analfabetismo } \\
\hline \multicolumn{12}{|c|}{ Muestra: Mayores a 15 años } \\
\hline Bolivia & & -0.07 & -0.02 & -0.09 & 0.02 & -0.03 & -0.13 & -0.08 & -0.03 & -0.07 & -0.04 \\
\hline Urbano & & 0.16 & 0.14 & -0.24 & 0.09 & -0.04 & -0.14 & -0.12 & -0.16 & -0.02 & 0.14 \\
\hline & Hombre & -0.16 & -0.1 & -0.02 & 0.01 & -0.01 & -0.1 & -0.07 & 0.02 & -0.08 & -0.12 \\
\hline & Mujer & 0.31 & 0.18 & -0.78 & 0.29 & -0.08 & -0.18 & 0.05 & -0.74 & 0.25 & 0.22 \\
\hline Rural & & 0.1 & 0.14 & -0.16 & 0.04 & -0.03 & -0.12 & -0.15 & -0.07 & -0.08 & 0.14 \\
\hline & Hombre & -0.12 & -0.29 & 0.06 & 0 & 0.04 & -0.26 & -0.04 & 0.02 & 0.04 & -0.27 \\
\hline & Mujer & -0.17 & -0.03 & -0.06 & 0.02 & -0.04 & -0.06 & -0.08 & 0.01 & -0.12 & -0.07 \\
\hline \multicolumn{12}{|c|}{ Muestra: Mayores a 25 años } \\
\hline Bolivia & & -0.08 & -0.01 & -0.08 & 0.02 & -0.02 & -0.16 & -0.08 & -0.02 & -0.07 & -0.05 \\
\hline Urbano & & 0.14 & 0.13 & -0.21 & 0.09 & -0.03 & -0.17 & -0.12 & -0.18 & 0.01 & 0.12 \\
\hline & Hombre & -0.14 & -0.07 & -0.01 & -0.02 & 0 & -0.12 & -0.07 & 0.06 & -0.12 & -0.12 \\
\hline & Mujer & 0.33 & 0.15 & -0.76 & 0.31 & -0.07 & -0.28 & 0.08 & -0.69 & 0.25 & 0.22 \\
\hline Rural & & 0.09 & 0.13 & -0.14 & 0.03 & -0.03 & -0.14 & -0.16 & -0.1 & -0.03 & 0.11 \\
\hline & Hombre & -0.1 & -0.26 & 0.09 & -0.05 & 0.05 & -0.27 & -0.01 & 0.03 & -0.01 & -0.3 \\
\hline & Mujer & -0.15 & -0.01 & -0.04 & 0 & -0.04 & -0.08 & -0.09 & 0.07 & -0.15 & -0.06 \\
\hline \multicolumn{12}{|c|}{ Panel B: Población Analfabeta } \\
\hline \multicolumn{12}{|c|}{ Muestra: Mayores a 15 años } \\
\hline Bolivia & & -0.03 & 0.01 & -0.05 & 0.04 & 0.02 & -0.06 & -0.07 & 0.01 & -0.03 & -0.01 \\
\hline
\end{tabular}




\begin{tabular}{|c|c|c|c|c|c|c|c|c|c|c|c|}
\hline \multicolumn{2}{|l|}{ Urbano } & 0.19 & 0.16 & -0.18 & 0.13 & 0.01 & -0.05 & -0.12 & -0.11 & 0.03 & 0.17 \\
\hline \multirow{5}{*}{ Rural } & Hombre & -0.12 & -0.07 & 0 & 0 & 0.02 & -0.06 & -0.05 & 0.06 & -0.05 & -0.12 \\
\hline & Mujer & 0.32 & 0.21 & -0.74 & 0.31 & -0.03 & -0.06 & 0.05 & -0.65 & 0.28 & 0.26 \\
\hline & & 0.16 & 0.14 & -0.09 & 0.08 & 0.02 & -0.05 & -0.16 & -0.03 & -0.03 & 0.15 \\
\hline & Hombre & -0.06 & -0.23 & 0.08 & -0.01 & 0.06 & -0.23 & -0.02 & 0.05 & 0.07 & -0.27 \\
\hline & Mujer & -0.14 & -0.01 & -0.03 & 0.01 & 0.01 & -0.02 & -0.05 & 0.06 & -0.08 & -0.07 \\
\hline \multicolumn{12}{|c|}{ Muestra: Mayores a 25 años } \\
\hline Bolivia & & -0.05 & 0.01 & -0.03 & 0.05 & 0.02 & -0.06 & -0.07 & 0.03 & -0.03 & -0.01 \\
\hline \multirow[t]{3}{*}{ Urbano } & & 0.17 & 0.15 & -0.15 & 0.12 & 0.01 & -0.05 & -0.12 & -0.11 & 0.04 & 0.18 \\
\hline & Hombre & -0.13 & -0.06 & 0.02 & 0.02 & 0.03 & -0.07 & -0.05 & 0.08 & -0.06 & -0.12 \\
\hline & Mujer & 0.35 & 0.17 & -0.64 & 0.31 & -0.03 & -0.13 & 0.09 & -0.62 & 0.3 & 0.27 \\
\hline \multirow[t]{3}{*}{ Rural } & & 0.14 & 0.14 & -0.08 & 0.08 & 0.02 & -0.04 & -0.17 & -0.03 & -0.01 & 0.15 \\
\hline & Hombre & -0.08 & -0.25 & 0.12 & 0.02 & 0.04 & -0.22 & 0 & 0.05 & 0.06 & -0.27 \\
\hline & Mujer & -0.16 & 0 & -0.02 & 0.02 & 0.02 & -0.02 & -0.06 & 0.09 & -0.1 & -0.07 \\
\hline
\end{tabular}

Fuente: Elaboración de los autores en base a la serie armonizada de Encuesta de Hogares de la Fundación ARU

Tabla 11: Evolución de las Tasas de Analfabetismo por Área y Departamento

\begin{tabular}{|c|c|c|c|c|c|c|c|}
\hline & & 1999-2002 & 2003-2005 & 2006-2009 & Dif 1 & Dif 2 & Dif Total \\
\hline \multicolumn{8}{|c|}{ Panel A: Muestra: Mayores a 15 años } \\
\hline Bolivia & & 14.0 & 12.3 & 9.7 & -1.7 & -2.6 & -0.9 \\
\hline & Chuquisaca & 26.7 & 22.7 & 17.6 & -4.0 & -5.1 & -1.1 \\
\hline & $\mathrm{La} \mathrm{Paz}$ & 11.4 & 9.0 & 8.5 & -2.4 & -0.5 & 1.9 \\
\hline & Cochabamba & 16.5 & 14.5 & 10.2 & -2.0 & -4.3 & -2.3 \\
\hline & Oruro & 12.1 & 10.7 & 9.3 & -1.4 & -1.4 & 0.0 \\
\hline & Potosi & 26.7 & 18.1 & 19.7 & -8.6 & 1.6 & 10.2 \\
\hline & Tarija & 15.9 & 13.2 & 8.5 & -2.7 & -4.7 & -2.0 \\
\hline & Santa Cruz & 7.7 & 8.4 & 5.6 & 0.7 & -2.8 & -3.5 \\
\hline & Beni & 6.7 & 6.5 & 5.4 & -0.2 & -1.1 & -0.9 \\
\hline & Pando & 6.8 & 7.0 & 5.1 & 0.2 & -1.9 & -2.1 \\
\hline Urbano & & 6.2 & 6.0 & 4.5 & -0.2 & -1.5 & -1.3 \\
\hline Rural & & 28.6 & 25.1 & 20.8 & -3.5 & -4.3 & -0.8 \\
\hline \multicolumn{8}{|c|}{ Panel B: Muestra: Mayores a 25 años } \\
\hline Bolivia & & 19.4 & 17.1 & 13.4 & -2.3 & -3.7 & -1.4 \\
\hline & Chuquisaca & 37.0 & 31.4 & 23.8 & -5.6 & -7.6 & -2.0 \\
\hline & $\mathrm{LaPaz}$ & 15.8 & 12.8 & 11.6 & -3.0 & -1.2 & 1.8 \\
\hline & Cochabamba & 22.9 & 20.0 & 14.0 & -2.9 & -6.0 & -3.1 \\
\hline & Oruro & 17.1 & 14.2 & 12.5 & -2.9 & -1.7 & 1.2 \\
\hline & Potosi & 35.2 & 25.3 & 26.4 & -9.9 & 1.1 & 11.0 \\
\hline & Tarija & 21.4 & 17.7 & 11.8 & -3.7 & -5.9 & -2.2 \\
\hline & Santa Cruz & 10.8 & 11.7 & 7.9 & 0.9 & -3.8 & -4.7 \\
\hline & Beni & 9.3 & 9.3 & 7.8 & 0.0 & -1.5 & -1.5 \\
\hline & Pando & 8.3 & 9.7 & 6.9 & 1.4 & -2.8 & -4.2 \\
\hline Urbano & & 8.9 & 8.7 & 6.3 & -0.2 & -2.4 & -2.2 \\
\hline Rural & & 37.3 & 32.4 & 27.1 & -4.9 & -5.3 & -0.4 \\
\hline
\end{tabular}

Fuente: Elaboración de los autores en base a la serie armonizada de Encuesta de Hogares de la Fundación ARU 
Tabla 12: Estados Financieros del PNA-Bolivia por Tipo de Gasto y Fuente de Financiamiento

\begin{tabular}{|c|c|c|c|c|c|}
\hline & 2006 & 2007 & 2008 & 2009 & Total \\
\hline \multicolumn{6}{|c|}{ Panel A. Gasto del PNA (Miles de Bs.) } \\
\hline Servicios no personales & $11,856.00$ & $12,578.10$ & $13,092.30$ & $2,822.50$ & $40,348.90$ \\
\hline Servicios Básicos & 78.4 & 305.8 & 497.5 & 197.7 & 1079.4 \\
\hline Servicios de Transporte y Seguros & 948.7 & 3854.1 & 3740.8 & 546.3 & 9089.9 \\
\hline Alquileres & 553.7 & 2188.8 & 2790.5 & 530.4 & 6063.5 \\
\hline Instalación, Mantenimiento y reparaciones & 5.2 & 70.1 & 134.4 & 86.3 & 296.0 \\
\hline Servicios Profesionales y Comerciales & 10270.0 & 6159.0 & 5929.0 & 1456.7 & 23814.7 \\
\hline Otros servicios no personales & 0.0 & 0.3 & 0.0 & 5.1 & 5.4 \\
\hline Materiales y suministros & 2488.5 & 11775.0 & 36691.7 & 2356.8 & 53312.1 \\
\hline Alimentos y Productos agroforestales & 1275.4 & 11019.4 & 30072.9 & 2022.3 & 44390.0 \\
\hline Productos de papel, cartón e impresos & 0.0 & 10.6 & 4781.5 & 8.2 & 4800.3 \\
\hline Textiles y vestuario & 0.0 & 276.1 & 138.5 & 72.1 & 486.6 \\
\hline $\begin{array}{l}\text { Combustibles, productos químicos, farmaceutic } \\
\text { y otros }\end{array}$ & 4.1 & 398.2 & 660.5 & 191.5 & 1254.3 \\
\hline Productos varios & 1209.0 & 70.7 & 1038.4 & 62.8 & 2380.9 \\
\hline Activos Reales & 109693.2 & 3779.4 & 48974.1 & 4.8 & 162451.5 \\
\hline Maquinaria y equipo & 109693.2 & 3779.4 & 48974.1 & 4.8 & 162451.5 \\
\hline Impuestos regalías y tasas & 0.0 & 137.4 & 11.2 & 6450.4 & 6599.0 \\
\hline Renta Interna & 0.0 & 0.0 & 0.0 & 6450.0 & 6450.0 \\
\hline Renta Aduanera & 0.0 & 137.2 & 6.8 & 0.0 & 144.1 \\
\hline Tasas, multas y otros & 0.0 & 0.2 & 4.3 & 0.4 & 4.9 \\
\hline Total & 124037.8 & 28269.9 & 98769.3 & 11634.5 & 262711.5 \\
\hline \multicolumn{6}{|c|}{ Panel B. Fuente de financiamiento del PNA (Miles de Bs.) } \\
\hline Donación Externa & $120,255.30$ & 351.3 & $54,892.00$ & $1,565.90$ & $177,064.40$ \\
\hline República de Cuba & $119,785.00$ & 0 & $54,538.40$ & 0 & $174,323.40$ \\
\hline Fondo Naciones Unidas para la infancia & 95.6 & 351.3 & 353.6 & 0 & 800.4 \\
\hline Otros organismos financiadores externos & 374.8 & 0 & 0 & 169.7 & 544.5 \\
\hline Otros organismos financiadores multilaterales & 0 & 0 & 0 & $1,396.10$ & $1,396.10$ \\
\hline Tesoro General de la Nación & $3,782.50$ & $27,918.60$ & $43,877.30$ & $10,068.60$ & $85,647.10$ \\
\hline Recursos de contravalor & $3,782.50$ & $16,829.50$ & $13,057.70$ & 851.2 & $34,520.90$ \\
\hline Tesoro General de la Nacion & 0 & $11,089.10$ & $9,627.90$ & $9,217.50$ & $29,934.40$ \\
\hline $\mathrm{NC}$ & 0 & 0 & $21,191.80$ & 0 & 21191.8 \\
\hline Total & $124,037.80$ & $28,269.90$ & $98,769.30$ & $11,634.50$ & $262,711.50$ \\
\hline
\end{tabular}

Fuente: Unidad Financiera del Ministerio de Educación y Cultura de Bolivia 
Tabla 13: Evaluación de Tendencias por Muestra según Especificación. Bolivia

\begin{tabular}{|c|c|c|c|c|c|c|c|c|}
\hline \multirow[b]{3}{*}{$\mathrm{t}$} & 1) & & & & 1) & & & \\
\hline & \multicolumn{4}{|c|}{ Panel A } & \multicolumn{4}{|c|}{ Panel B } \\
\hline & $-0.059 * * *$ & $-0.050 * * *$ & $-0.049 * * *$ & $-0.050 * * *$ & $-0.059 * * *$ & $-0.056^{* * *}$ & $-0.056^{* * *}$ & $-0.056 * * *$ \\
\hline & $(0.00)$ & $(0.01)$ & $(0.01)$ & $(0.01)$ & $(0.00)$ & $(0.01)$ & $(0.01)$ & $(0.01)$ \\
\hline \multirow[t]{2}{*}{ D } & & -0.073 & & -0.054 & & -0.03 & & -0.023 \\
\hline & & $(0.05)$ & & $(0.03)$ & & $(0.04)$ & & $(0.04)$ \\
\hline \multirow[t]{2}{*}{ Dt } & & & -0.009 & -0.003 & & & -0.003 & -0.001 \\
\hline & & & $(0.01)$ & $(0.01)$ & & & $(0.01)$ & $(0.01)$ \\
\hline \multirow[t]{2}{*}{ cons } & $-1.707 * * *$ & $-1.727 * * *$ & $-1.730 * * *$ & $-1.728 * * *$ & $-1.707 * * *$ & $-1.715^{* * *}$ & $-1.716^{* * *}$ & $-1.715^{* * *}$ \\
\hline & $(0.02)$ & $(0.01)$ & $(0.02)$ & $(0.02)$ & $(0.02)$ & $(0.02)$ & $(0.02)$ & $(0.02)$ \\
\hline $\mathrm{r} 2$ & 0.97 & 0.98 & 0.98 & 0.98 & 0.97 & 0.972 & 0.972 & 0.972 \\
\hline \multirow[t]{2}{*}{$\mathrm{N}$} & 11 & 11 & 11 & 11 & 11 & 11 & 11 & 11 \\
\hline & \multicolumn{4}{|c|}{ Panel C } & \multicolumn{4}{|c|}{ Panel D } \\
\hline \multirow[t]{2}{*}{$\mathrm{t}$} & $-0.063 * * *$ & $-0.052 * * *$ & $-0.051 * *$ & $-0.051 * *$ & $-0.063 * * *$ & $-0.060 * * *$ & $-0.059 * * *$ & $-0.059 * * *$ \\
\hline & $(0.00)$ & $(0.01)$ & $(0.01)$ & $(0.01)$ & $(0.00)$ & $(0.01)$ & $(0.01)$ & $(0.01)$ \\
\hline \multirow[t]{2}{*}{ D } & & -0.089 & & -0.055 & & -0.03 & & 0.053 \\
\hline & & $(0.06)$ & & $(0.06)$ & & $(0.06)$ & & $(0.04)$ \\
\hline \multirow[t]{2}{*}{ Dt } & & & -0.011 & -0.004 & & & -0.004 & -0.009 \\
\hline & & & $(0.01)$ & $(0.01)$ & & & $(0.01)$ & $(0.01)$ \\
\hline \multirow[t]{2}{*}{ cons } & $-1.309 * * *$ & $-1.333 * * *$ & $-1.337 * * *$ & $-1.335^{* * *}$ & $-1.309 * * *$ & $-1.317 * * *$ & $-1.318^{* * *}$ & $-1.319 * * *$ \\
\hline & $(0.02)$ & $(0.02)$ & $(0.02)$ & $(0.02)$ & $(0.02)$ & $(0.02)$ & $(0.02)$ & $(0.02)$ \\
\hline $\mathrm{r} 2$ & 0.958 & 0.972 & 0.971 & 0.972 & 0.958 & 0.96 & 0.96 & 0.96 \\
\hline $\mathrm{N}$ & 11 & 11 & 11 & 11 & 11 & 11 & 11 & 11 \\
\hline
\end{tabular}


Tabla 14: Evaluación de Tendencias por Área y Sego según Especificación (Muestra: Mayores a 15 años)

\begin{tabular}{|c|c|c|c|c|c|c|c|c|}
\hline & $(1)$ & $(2)$ & (3) & (4) & (1) & $(2)$ & (3) & (4) \\
\hline \multicolumn{9}{|c|}{ Panel A. Area Urbana } \\
\hline \multirow[t]{2}{*}{$\mathrm{t}$} & $-0.042 *$ & -0.003 & 0 & -0.002 & $-0.042^{*}$ & -0.017 & -0.019 & -0.021 \\
\hline & $(0.02)$ & $(0.02)$ & $(0.03)$ & $(0.03)$ & $(0.02)$ & $(0.02)$ & $(0.02)$ & $(0.03)$ \\
\hline \multirow[t]{2}{*}{$\mathrm{D}$} & & $-0.310^{*}$ & & -0.27 & & -0.231 & & $-1.025^{* *}$ \\
\hline & & $(0.13)$ & & $(0.39)$ & & $(0.14)$ & & $(0.27)$ \\
\hline \multirow[t]{2}{*}{ Dt } & & & -0.037 & -0.005 & & & -0.023 & $0.091^{*}$ \\
\hline & & & $(0.02)$ & $(0.05)$ & & & $(0.02)$ & $(0.04)$ \\
\hline \multirow[t]{2}{*}{ cons } & $-2.638 * * *$ & $-2.723 * * *$ & $-2.733 * * *$ & $-2.725^{* * *}$ & $-2.638^{* * *}$ & $-2.701 * * *$ & $-2.697 * * *$ & $-2.687 * * *$ \\
\hline & $(0.10)$ & $(0.10)$ & $(0.10)$ & $(0.11)$ & $(0.10)$ & $(0.11)$ & $(0.11)$ & $(0.12)$ \\
\hline $\mathrm{r} 2$ & 0.525 & 0.72 & 0.708 & 0.721 & 0.525 & 0.649 & 0.624 & 0.691 \\
\hline $\mathrm{N}$ & 11 & 11 & 11 & 11 & 11 & 11 & 11 & 11 \\
\hline \multicolumn{9}{|c|}{ Panel B. Urbano Hombre } \\
\hline \multirow[t]{2}{*}{$\mathrm{t}$} & -0.041 & -0.004 & -0.004 & -0.008 & -0.041 & 0 & -0.007 & -0.013 \\
\hline & $(0.03)$ & $(0.04)$ & $(0.04)$ & $(0.04)$ & $(0.03)$ & $(0.03)$ & $(0.03)$ & $(0.04)$ \\
\hline \multirow[t]{2}{*}{$\mathrm{D}$} & & -0.293 & & -0.508 & & -0.371 & & $-2.829^{* * *}$ \\
\hline & & $(0.25)$ & & $(1.11)$ & & $(0.21)$ & & $(0.20)$ \\
\hline \multirow[t]{2}{*}{$\mathrm{Dt}$} & & & -0.032 & 0.028 & & & -0.033 & $0.281 * * *$ \\
\hline & & & $(0.03)$ & $(0.13)$ & & & $(0.02)$ & $(0.04)$ \\
\hline \multirow[t]{2}{*}{ cons } & $-3.720 * * *$ & $-3.800 * * *$ & $-3.802 * * *$ & $-3.787 * * *$ & $-3.720 * * *$ & $-3.821 * * *$ & $-3.805^{* * *}$ & $-3.776 * * *$ \\
\hline & $(0.16)$ & $(0.18)$ & $(0.18)$ & $(0.20)$ & $(0.16)$ & $(0.17)$ & $(0.18)$ & $(0.19)$ \\
\hline $\mathrm{r} 2$ & 0.246 & 0.333 & 0.315 & 0.338 & 0.246 & 0.406 & 0.348 & 0.608 \\
\hline $\mathrm{N}$ & 11 & 11 & 11 & 11 & 11 & 11 & 11 & 11 \\
\hline \multicolumn{9}{|c|}{ Panel C. Urbano Mujer } \\
\hline \multirow[t]{2}{*}{ t } & $-0.043^{*}$ & -0.004 & -0.001 & -0.003 & $-0.043^{*}$ & -0.021 & -0.023 & -0.024 \\
\hline & $(0.01)$ & $(0.02)$ & $(0.02)$ & $(0.02)$ & $(0.01)$ & $(0.02)$ & $(0.02)$ & $(0.03)$ \\
\hline \multirow[t]{2}{*}{$\mathrm{D}$} & & $-0.304 *$ & & -0.254 & & -0.194 & & -0.716 \\
\hline & & $(0.10)$ & & $(0.28)$ & & $(0.13)$ & & (0.34) \\
\hline \multirow[t]{2}{*}{ Dt } & & & $-0.037^{*}$ & -0.007 & & & -0.02 & 0.06 \\
\hline & & & $(0.01)$ & $(0.04)$ & & & $(0.02)$ & $(0.04)$ \\
\hline \multirow[t]{2}{*}{ cons } & $-2.151 * * *$ & $-2.234 * * *$ & $-2.245^{* * *}$ & $-2.237 * * *$ & $-2.151 * * *$ & $-2.204 * * *$ & $-2.202 * * *$ & $-2.195 * * *$ \\
\hline & $(0.09)$ & $(0.08)$ & $(0.08)$ & $(0.09)$ & $(0.09)$ & $(0.10)$ & $(0.10)$ & $(0.11)$ \\
\hline $\mathrm{r} 2$ & 0.58 & 0.786 & 0.774 & 0.786 & 0.58 & 0.676 & 0.66 & 0.696 \\
\hline $\mathrm{N}$ & 11 & 11 & 11 & 11 & 11 & 11 & 11 & 11 \\
\hline \multicolumn{9}{|c|}{ Panel D. Area Rural } \\
\hline \multirow[t]{2}{*}{$\mathrm{t}$} & $-0.064 * * *$ & $-0.068 * *$ & $-0.068^{* *}$ & $-0.067^{* *}$ & $-0.064 * * *$ & $-0.070^{* * *}$ & $-0.069^{* * *}$ & $-0.068 * *$ \\
\hline & $(0.01)$ & $(0.01)$ & $(0.02)$ & $(0.02)$ & $(0.01)$ & $(0.01)$ & $(0.01)$ & $(0.01)$ \\
\hline \multirow[t]{2}{*}{$\mathrm{D}$} & & 0.029 & & 0.082 & & 0.053 & & $0.494 * *$ \\
\hline & & $(0.07)$ & & $(0.22)$ & & $(0.06)$ & & $(0.10)$ \\
\hline \multirow[t]{2}{*}{$\mathrm{Dt}$} & & & 0.003 & -0.007 & & & 0.004 & $-0.050 *$ \\
\hline & & & $(0.01)$ & $(0.03)$ & & & $(0.01)$ & $(0.02)$ \\
\hline \multirow[t]{2}{*}{ cons } & $-0.795^{* * *}$ & $-0.787 * * *$ & $-0.788^{* * *}$ & $-0.790 * * *$ & $-0.795 * * *$ & $-0.781 * * *$ & $-0.784 * * *$ & $-0.789 * * *$ \\
\hline & $(0.06)$ & $(0.07)$ & $(0.07)$ & $(0.08)$ & $(0.06)$ & $(0.06)$ & $(0.07)$ & $(0.07)$ \\
\hline
\end{tabular}




\begin{tabular}{|c|c|c|c|c|c|c|c|c|}
\hline $\mathrm{r} 2$ & 0.913 & 0.914 & 0.913 & 0.914 & 0.913 & 0.918 & 0.915 & 0.927 \\
\hline $\mathrm{N}$ & 11 & 11 & 11 & 11 & 11 & 11 & 11 & 11 \\
\hline \multicolumn{9}{|c|}{ Panel E. Rural Hombre } \\
\hline \multirow[t]{2}{*}{$\mathrm{t}$} & $-0.064 * * *$ & $-0.070 * *$ & $-0.071 * *$ & $-0.072 *$ & $-0.064 * * *$ & $-0.075 * * *$ & $-0.074 * * *$ & $-0.074 * *$ \\
\hline & $(0.01)$ & $(0.02)$ & $(0.02)$ & $(0.02)$ & $(0.01)$ & $(0.01)$ & $(0.01)$ & $(0.02)$ \\
\hline \multirow[t]{2}{*}{$\mathrm{D}$} & & 0.042 & & -0.065 & & 0.102 & & 0.44 \\
\hline & & $(0.11)$ & & $(0.34)$ & & $(0.08)$ & & $(0.42)$ \\
\hline \multirow[t]{2}{*}{ Dt } & & & 0.006 & 0.014 & & & 0.01 & -0.039 \\
\hline & & & $(0.01)$ & $(0.05)$ & & & $(0.01)$ & $(0.05)$ \\
\hline \multirow[t]{2}{*}{ cons } & $-1.589 * * *$ & $-1.577 * * *$ & $-1.573^{* * *}$ & $-1.571^{* * *}$ & $-1.589^{* * *}$ & $-1.561 * * *$ & $-1.563 * * *$ & $-1.567 * * *$ \\
\hline & $(0.07)$ & $(0.08)$ & $(0.08)$ & $(0.09)$ & $(0.07)$ & $(0.07)$ & $(0.07)$ & $(0.08)$ \\
\hline r2 & 0.818 & 0.821 & 0.822 & 0.822 & 0.818 & 0.834 & 0.831 & 0.839 \\
\hline $\mathrm{N}$ & 11 & 11 & 11 & 11 & 11 & 11 & 11 & 11 \\
\hline \multicolumn{9}{|c|}{ Panel F. Rural Mujer } \\
\hline \multirow[t]{2}{*}{$\mathrm{t}$} & $-0.075 * * *$ & $-0.077 * *$ & $-0.076^{* *}$ & $-0.075^{* *}$ & $-0.075^{* * *}$ & $-0.079 * * *$ & $-0.077 * * *$ & $-0.076 * *$ \\
\hline & $(0.01)$ & $(0.02)$ & $(0.02)$ & $(0.02)$ & $(0.01)$ & $(0.01)$ & $(0.01)$ & $(0.02)$ \\
\hline \multirow[t]{2}{*}{$\mathrm{D}$} & & 0.013 & & 0.128 & & 0.031 & & $0.511 * *$ \\
\hline & & $(0.07)$ & & $(0.20)$ & & $(0.07)$ & & $(0.12)$ \\
\hline \multirow[t]{2}{*}{$\mathrm{Dt}$} & & & 0 & -0.015 & & & 0.002 & $-0.055^{*}$ \\
\hline & & & $(0.01)$ & $(0.03)$ & & & $(0.01)$ & $(0.02)$ \\
\hline \multirow[t]{2}{*}{ cons } & $-0.194 * *$ & $-0.191 *$ & $-0.194 *$ & -0.198 & $-0.194 * *$ & $-0.186^{*}$ & $-0.189 *$ & $-0.195 *$ \\
\hline & $(0.06)$ & $(0.07)$ & $(0.08)$ & $(0.09)$ & $(0.06)$ & $(0.07)$ & $(0.07)$ & $(0.08)$ \\
\hline $\mathrm{r} 2$ & 0.933 & 0.933 & 0.933 & 0.934 & 0.933 & 0.934 & 0.933 & 0.942 \\
\hline $\mathrm{N}$ & 11 & 11 & 11 & 11 & 11 & 11 & 11 & 11 \\
\hline
\end{tabular}

Fuente: Elaboración de los autores en base a la serie armonizada de la Encuesta de Hogares de la Fundación ARU 
Tabla 15: Evaluación de Tendencias por Área y Sexo según Especificación (Muestra: Mayores a 25 años)

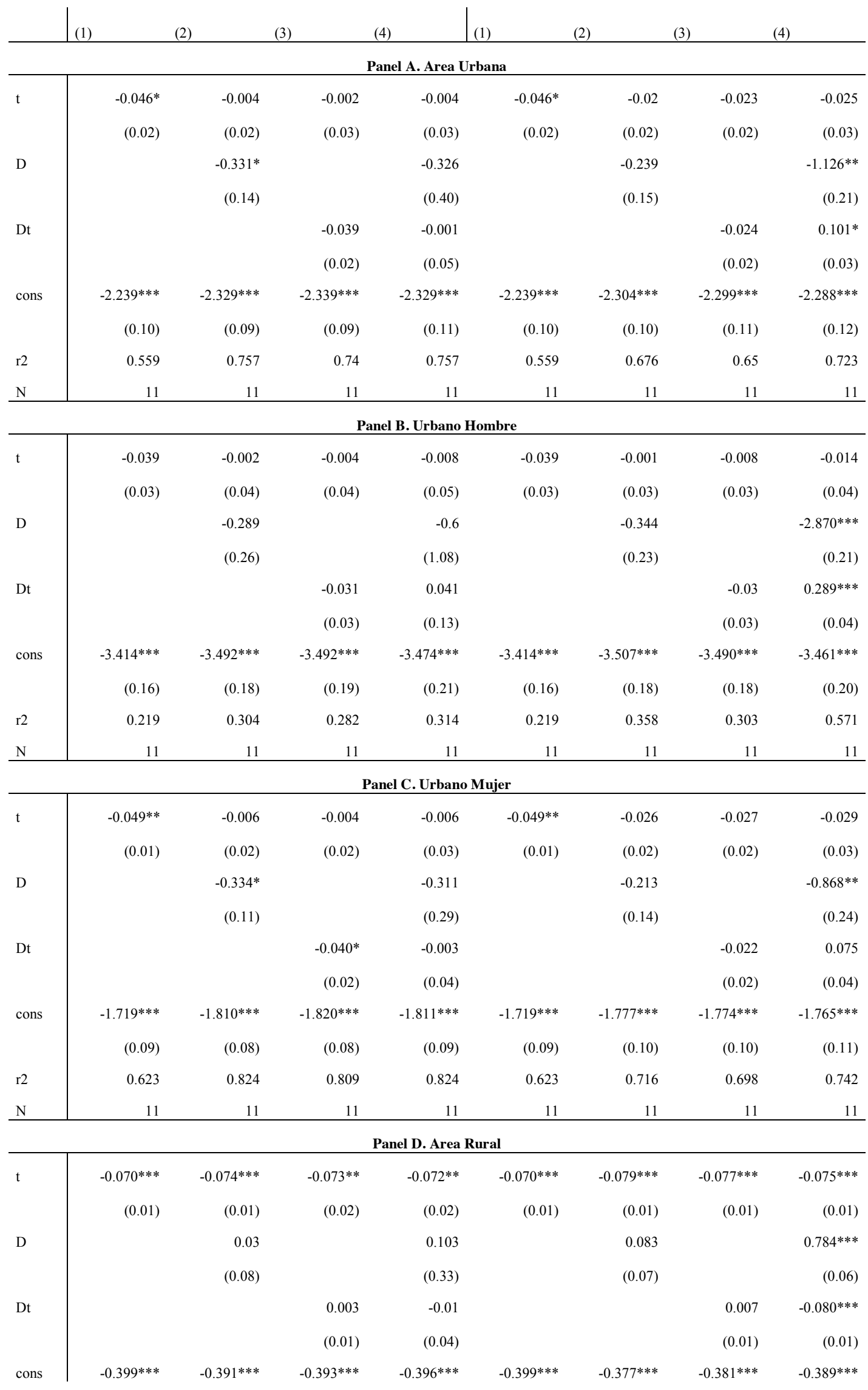




\begin{tabular}{|c|c|c|c|c|c|c|c|c|}
\hline & $(0.05)$ & $(0.06)$ & $(0.06)$ & $(0.07)$ & $(0.05)$ & $(0.05)$ & $(0.05)$ & $(0.06)$ \\
\hline $\mathrm{r} 2$ & 0.921 & 0.923 & 0.922 & 0.923 & 0.921 & 0.932 & 0.927 & 0.953 \\
\hline $\mathrm{N}$ & 11 & 11 & 11 & 11 & 11 & 11 & 11 & 11 \\
\hline
\end{tabular}

Panel E. Rural Hombre

\begin{tabular}{|c|c|c|c|c|c|c|c|c|}
\hline \multicolumn{9}{|c|}{ Panel E. Rural Hombre } \\
\hline \multirow[t]{2}{*}{$\mathrm{t}$} & $-0.067 * * *$ & $-0.075^{* *}$ & $-0.075 * *$ & $-0.074 * *$ & $-0.067 * * *$ & $-0.078 * * *$ & $-0.076 * * *$ & $-0.074 * *$ \\
\hline & $(0.01)$ & $(0.02)$ & $(0.02)$ & $(0.02)$ & $(0.01)$ & $(0.01)$ & $(0.01)$ & $(0.01)$ \\
\hline \multirow[t]{2}{*}{$\mathrm{D}$} & & 0.067 & & 0.121 & & 0.103 & & 0.803 \\
\hline & & $(0.11)$ & & $(0.41)$ & & $(0.09)$ & & $(0.40)$ \\
\hline \multirow[t]{2}{*}{ Dt } & & & 0.007 & -0.007 & & & 0.009 & -0.08 \\
\hline & & & $(0.02)$ & $(0.05)$ & & & $(0.01)$ & $(0.05)$ \\
\hline \multirow[t]{2}{*}{ cons } & $-1.252 * * *$ & $-1.234 * * *$ & $-1.234 * * *$ & $-1.237 * * *$ & $-1.252 * * *$ & $-1.224 * * *$ & $-1.229 * * *$ & $-1.237 * * *$ \\
\hline & $(0.06)$ & $(0.07)$ & $(0.07)$ & $(0.08)$ & $(0.06)$ & $(0.07)$ & $(0.07)$ & $(0.07)$ \\
\hline $\mathrm{r} 2$ & 0.825 & 0.831 & 0.83 & 0.831 & 0.825 & 0.841 & 0.835 & 0.862 \\
\hline $\mathrm{N}$ & 11 & 11 & 11 & 11 & 11 & 11 & 11 & 11 \\
\hline \multicolumn{9}{|c|}{ Panel F. Rural Mujer } \\
\hline \multirow[t]{2}{*}{$\mathrm{t}$} & $-0.086 * * *$ & $-0.085^{* * *}$ & $-0.085^{* *}$ & $-0.085 * *$ & $-0.086^{* * *}$ & $-0.095 * * *$ & $-0.093 * * *$ & $-0.092 * * *$ \\
\hline & $(0.01)$ & $(0.02)$ & $(0.02)$ & $(0.02)$ & $(0.01)$ & $(0.01)$ & $(0.01)$ & $(0.02)$ \\
\hline \multirow[t]{2}{*}{$\mathrm{D}$} & & -0.007 & & 0.02 & & 0.08 & & $0.731^{*}$ \\
\hline & & $(0.09)$ & & $(0.35)$ & & $(0.08)$ & & $(0.21)$ \\
\hline \multirow[t]{2}{*}{ Dt } & & & -0.001 & -0.004 & & & 0.007 & $-0.074 *$ \\
\hline & & & $(0.01)$ & $(0.04)$ & & & $(0.01)$ & $(0.03)$ \\
\hline \multirow[t]{2}{*}{ cons } & $0.297 * * *$ & $0.295^{* *}$ & $0.294 * *$ & $0.293^{*}$ & $0.297 * * *$ & $0.319^{* *}$ & $0.315^{* *}$ & $0.307 * *$ \\
\hline & $(0.06)$ & $(0.08)$ & $(0.08)$ & $(0.09)$ & $(0.06)$ & $(0.07)$ & $(0.07)$ & $(0.08)$ \\
\hline $\mathrm{r} 2$ & 0.934 & 0.934 & 0.934 & 0.934 & 0.934 & 0.94 & 0.938 & 0.952 \\
\hline $\mathrm{N}$ & 11 & 11 & 11 & 11 & 11 & 11 & 11 & 11 \\
\hline
\end{tabular}

Fuente: Elaboración de los autores en base a la serie armonizada de la Encuesta de Hogares de la Fundación ARU 
Tabla 16: Evaluación de Tendencias por Muestra según Especificación. Bolivia (Con Dummie para los años 2003-2004)

\begin{tabular}{|c|c|c|c|c|c|c|c|c|}
\hline & & & & & 1) & & & \\
\hline \multirow[b]{2}{*}{ t } & \multicolumn{4}{|c|}{ Panel A } & \multicolumn{4}{|c|}{ Panel B } \\
\hline & $-0.059^{* * *}$ & $-0.057 * * *$ & $-0.057 * * *$ & $-0.058^{* * *}$ & $-0.059^{* * *}$ & $-0.060^{* * *}$ & $-0.060^{* * *}$ & $-0.060^{* * *}$ \\
\hline & $(0.00)$ & $(0.00)$ & $(0.00)$ & $(0.00)$ & $(0.00)$ & $(0.00)$ & $(0.00)$ & $(0.00)$ \\
\hline \multirow[t]{2}{*}{ De } & $0.078^{* * *}$ & $0.071^{* *}$ & $0.073^{* *}$ & $0.073^{* *}$ & $0.078^{* * * *}$ & $0.080^{* *}$ & $0.081 * *$ & $0.081 * *$ \\
\hline & $(0.01)$ & $(0.02)$ & $(0.02)$ & $(0.02)$ & $(0.01)$ & $(0.02)$ & $(0.02)$ & $(0.02)$ \\
\hline \multirow[t]{2}{*}{$\mathrm{D}$} & & -0.017 & & -0.057 & & 0.01 & & -0.017 \\
\hline & & $(0.02)$ & & $(0.03)$ & & $(0.02)$ & & $(0.04)$ \\
\hline \multirow[t]{2}{*}{$\mathrm{Dt}$} & & & -0.001 & 0.005 & & & 0.001 & 0.003 \\
\hline & & & $(0.00)$ & $(0.00)$ & & & $(0.00)$ & $(0.01)$ \\
\hline \multirow[t]{2}{*}{ cons } & $-1.725^{* * *}$ & $-1.728 * * *$ & $-1.727 * * *$ & $-1.726 * * *$ & $-1.725^{* * *}$ & $-1.723^{* * *}$ & $-1.722 * * *$ & $-1.722 * * *$ \\
\hline & $(0.01)$ & $(0.01)$ & $(0.01)$ & $(0.01)$ & $(0.01)$ & $(0.01)$ & $(0.01)$ & $(0.01)$ \\
\hline $\mathrm{r} 2$ & 0.994 & 0.995 & 0.995 & 0.995 & 0.994 & 0.995 & 0.995 & 0.995 \\
\hline \multirow[t]{2}{*}{$\mathrm{N}$} & 11 & 11 & 11 & 11 & 11 & 11 & 11 & 11 \\
\hline & \multicolumn{4}{|c|}{ Panel C } & \multicolumn{4}{|c|}{ Panel D } \\
\hline \multirow[t]{2}{*}{ t } & $-0.062^{* * *}$ & $-0.060^{* * *}$ & $-0.061^{* * *}$ & $-0.061^{* * *}$ & $-0.062^{* * *}$ & $-0.065^{* * *}$ & $-0.064^{* * *}$ & $-0.064 * * *$ \\
\hline & $(0.00)$ & $(0.00)$ & $(0.00)$ & $(0.00)$ & $(0.00)$ & $(0.00)$ & $(0.00)$ & $(0.00)$ \\
\hline \multirow[t]{2}{*}{$\mathrm{De}$} & $0.099 * * *$ & $0.093 * *$ & $0.095^{* *}$ & $0.095^{* *}$ & $0.099^{* * *}$ & $0.105^{* *}$ & $0.105 * *$ & $0.105^{* *}$ \\
\hline & $(0.02)$ & $(0.02)$ & $(0.02)$ & $(0.02)$ & $(0.02)$ & $(0.02)$ & $(0.02)$ & $(0.02)$ \\
\hline \multirow[t]{2}{*}{$\mathrm{D}$} & & -0.016 & & -0.059 & & 0.022 & & 0.061 \\
\hline & & $(0.02)$ & & $(0.06)$ & & $(0.02)$ & & $(0.04)$ \\
\hline \multirow[t]{2}{*}{$\mathrm{Dt}$} & & & -0.001 & 0.006 & & & 0.002 & -0.005 \\
\hline & & & $(0.00)$ & $(0.01)$ & & & $(0.00)$ & $(0.01)$ \\
\hline \multirow[t]{2}{*}{ cons } & $-1.332^{* * *}$ & $-1.335^{* * *}$ & $-1.334 * * *$ & $-1.332^{* * *}$ & $-1.332 * * *$ & $-1.327 * * *$ & $-1.327 * * *$ & $-1.328 * * *$ \\
\hline & $(0.01)$ & $(0.01)$ & $(0.01)$ & $(0.02)$ & $(0.01)$ & $(0.01)$ & $(0.01)$ & $(0.02)$ \\
\hline $\mathrm{r} 2$ & 0.993 & 0.993 & 0.993 & 0.994 & 0.993 & 0.994 & 0.994 & 0.994 \\
\hline $\mathrm{N}$ & 11 & 11 & 11 & 11 & 11 & 11 & 11 & 11 \\
\hline
\end{tabular}

Fuente: Elaboración de los autores en base a la serie armonizada de la Encuesta de Hogares de la Fundación ARU 
Tabla 17: Evaluación de Tendencias por Área y Sexo según Especificación con Dummie para los años 2003-2004 (Muestra: Mayores a 15 años)

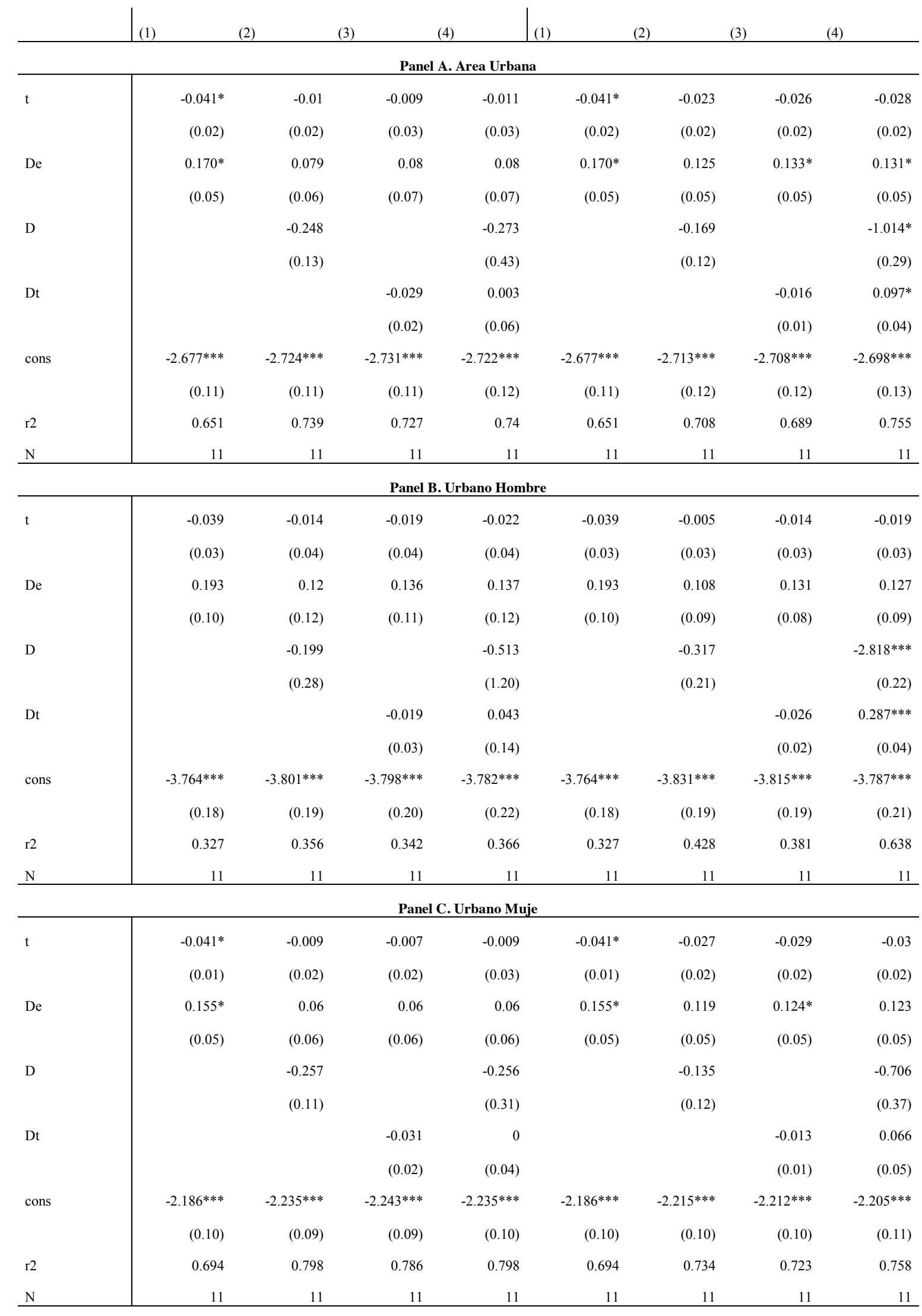

Panel D. Area Rural

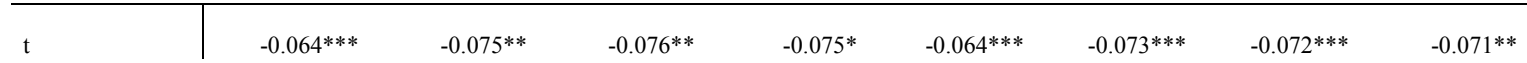




\begin{tabular}{|c|c|c|c|c|c|c|c|c|}
\hline & $(0.01)$ & $(0.02)$ & $(0.02)$ & $(0.02)$ & $(0.01)$ & $(0.01)$ & $(0.01)$ & $(0.02)$ \\
\hline \multirow[t]{2}{*}{$\mathrm{De}$} & 0.044 & 0.077 & 0.078 & 0.078 & 0.044 & 0.068 & 0.064 & 0.065 \\
\hline & $(0.04)$ & $(0.05)$ & $(0.05)$ & $(0.06)$ & $(0.04)$ & $(0.04)$ & $(0.04)$ & $(0.04)$ \\
\hline \multirow[t]{2}{*}{$\mathrm{D}$} & & 0.09 & & 0.08 & & 0.087 & & $0.499 * *$ \\
\hline & & $(0.10)$ & & $(0.23)$ & & $(0.06)$ & & $(0.10)$ \\
\hline \multirow[t]{2}{*}{$\mathrm{Dt}$} & & & 0.011 & 0.001 & & & 0.008 & $-0.047^{*}$ \\
\hline & & & $(0.01)$ & $(0.03)$ & & & $(0.01)$ & $(0.02)$ \\
\hline \multirow[t]{2}{*}{ cons } & $-0.805^{* * *}$ & $-0.788^{* * *}$ & $-0.785^{* * *}$ & $-0.788^{* * *}$ & $-0.805^{* * *}$ & $-0.787 * * *$ & $-0.789 * * *$ & $-0.794 * * *$ \\
\hline & $(0.06)$ & $(0.07)$ & $(0.07)$ & $(0.08)$ & $(0.06)$ & $(0.07)$ & $(0.07)$ & $(0.08)$ \\
\hline r2 & 0.919 & 0.928 & 0.927 & 0.928 & 0.919 & 0.93 & 0.927 & 0.939 \\
\hline $\mathrm{N}$ & 11 & 11 & 11 & 11 & 11 & 11 & 11 & 11 \\
\hline \multicolumn{9}{|c|}{ Panel E. Rural Hombre } \\
\hline \multirow[t]{2}{*}{$t$} & $-0.063^{* * *}$ & $-0.082^{* *}$ & $-0.087 * *$ & $-0.087^{* *}$ & $-0.063^{* * *}$ & $-0.081 * * *$ & $-0.080^{* * *}$ & $-0.079 * *$ \\
\hline & $(0.01)$ & $(0.02)$ & $(0.02)$ & $(0.02)$ & $(0.01)$ & $(0.01)$ & $(0.01)$ & $(0.02)$ \\
\hline \multirow[t]{2}{*}{$\mathrm{De}$} & 0.08 & 0.135 & 0.146 & 0.146 & 0.08 & 0.124 & 0.121 & 0.122 \\
\hline & $(0.06)$ & $(0.07)$ & $(0.08)$ & $(0.08)$ & $(0.06)$ & $(0.07)$ & $(0.07)$ & $(0.07)$ \\
\hline \multirow[t]{2}{*}{$\mathrm{D}$} & & 0.148 & & -0.071 & & 0.163 & & 0.45 \\
\hline & & $(0.09)$ & & $(0.37)$ & & $(0.07)$ & & $(0.45)$ \\
\hline \multirow[t]{2}{*}{$\mathrm{Dt}$} & & & 0.021 & 0.03 & & & 0.017 & -0.033 \\
\hline & & & $(0.01)$ & $(0.05)$ & & & $(0.01)$ & $(0.05)$ \\
\hline \multirow[t]{2}{*}{ cons } & $-1.607 * * *$ & $-1.579 * * *$ & $-1.568 * * *$ & $-1.566 * * *$ & $-1.607 * * *$ & $-1.572 * * *$ & $-1.573 * * *$ & $-1.577^{* * *}$ \\
\hline & $(0.08)$ & $(0.08)$ & $(0.08)$ & $(0.08)$ & $(0.08)$ & $(0.08)$ & $(0.08)$ & $(0.09)$ \\
\hline r2 & 0.837 & 0.858 & 0.864 & 0.865 & 0.837 & 0.873 & 0.868 & 0.877 \\
\hline $\mathrm{N}$ & 11 & 11 & 11 & 11 & 11 & 11 & 11 & 11 \\
\hline \multicolumn{9}{|c|}{ Panel F. Rural Mujer } \\
\hline \multirow[t]{2}{*}{$\mathrm{t}$} & $-0.075^{* * *}$ & $-0.083^{* *}$ & $-0.082^{* *}$ & $-0.081 *$ & $-0.075^{* * *}$ & $-0.081 * * *$ & $-0.080^{* *}$ & $-0.079 * *$ \\
\hline & $(0.01)$ & $(0.02)$ & $(0.02)$ & $(0.03)$ & $(0.01)$ & $(0.02)$ & $(0.02)$ & $(0.02)$ \\
\hline \multirow[t]{2}{*}{$\mathrm{De}$} & 0.042 & 0.066 & 0.063 & 0.063 & 0.042 & 0.058 & 0.054 & 0.055 \\
\hline & $(0.03)$ & $(0.05)$ & $(0.05)$ & $(0.05)$ & $(0.03)$ & $(0.03)$ & $(0.03)$ & $(0.03)$ \\
\hline \multirow[t]{2}{*}{$\mathrm{D}$} & & 0.065 & & 0.126 & & 0.06 & & $0.515^{* *}$ \\
\hline & & $(0.11)$ & & $(0.21)$ & & $(0.08)$ & & $(0.13)$ \\
\hline \multirow[t]{2}{*}{$\mathrm{Dt}$} & & & 0.007 & -0.008 & & & 0.005 & $-0.052^{*}$ \\
\hline & & & $(0.02)$ & $(0.03)$ & & & $(0.01)$ & $(0.02)$ \\
\hline \multirow[t]{2}{*}{ cons } & $-0.204^{*}$ & $-0.192 *$ & -0.192 & -0.195 & $-0.204 *$ & $-0.191 *$ & $-0.194 *$ & -0.199 \\
\hline & $(0.07)$ & $(0.08)$ & $(0.08)$ & $(0.10)$ & $(0.07)$ & $(0.08)$ & $(0.08)$ & $(0.09)$ \\
\hline $\mathrm{r} 2$ & 0.937 & 0.94 & 0.939 & 0.941 & 0.937 & 0.941 & 0.939 & 0.949 \\
\hline $\mathrm{N}$ & 11 & 11 & 11 & 11 & 11 & 11 & 11 & 11 \\
\hline
\end{tabular}

Fuente: Elaboración de los autores en base a la serie armonizada de la Encuesta de Hogares de la Fundación ARU 
Tabla 18: Evaluación de Tendencias por Área y Sexo según Especificación con Dummie para los años 2003-2004 (Muestra: Mayores a 25 años)

\begin{tabular}{|c|c|c|c|c|c|c|c|c|}
\hline & (1) & (2) & (3) & 4) & (1) & (2) & (3) & \\
\hline \multicolumn{9}{|c|}{ Panel A. Area Urbana } \\
\hline \multirow[t]{2}{*}{$\mathrm{t}$} & $-0.045^{*}$ & -0.013 & -0.013 & -0.015 & $-0.045^{*}$ & -0.027 & -0.03 & -0.032 \\
\hline & $(0.02)$ & $(0.02)$ & $(0.02)$ & $(0.03)$ & $(0.02)$ & $(0.02)$ & $(0.02)$ & $(0.02)$ \\
\hline \multirow[t]{2}{*}{$\mathrm{De}$} & $0.193^{* *}$ & 0.1 & 0.103 & 0.104 & $0.193 * *$ & $0.149^{*}$ & $0.157^{*}$ & $0.156^{*}$ \\
\hline & $(0.05)$ & $(0.06)$ & $(0.06)$ & $(0.07)$ & $(0.05)$ & $(0.05)$ & $(0.05)$ & $(0.05)$ \\
\hline \multirow[t]{2}{*}{$\mathrm{D}$} & & -0.252 & & -0.33 & & -0.164 & & $-1.113^{* *}$ \\
\hline & & $(0.14)$ & & $(0.43)$ & & $(0.12)$ & & $(0.23)$ \\
\hline \multirow[t]{2}{*}{$\mathrm{Dt}$} & & & -0.029 & 0.011 & & & -0.015 & $0.109 *$ \\
\hline & & & $(0.02)$ & $(0.06)$ & & & $(0.01)$ & $(0.03)$ \\
\hline \multirow[t]{2}{*}{ cons } & $-2.282 * * *$ & $-2.330^{* * *}$ & $-2.335^{* * *}$ & $-2.325^{* * *}$ & $-2.282^{* * *}$ & $-2.317 * * *$ & $-2.312^{* * *}$ & $-2.301 * * *$ \\
\hline & $(0.10)$ & $(0.10)$ & $(0.10)$ & $(0.12)$ & $(0.10)$ & $(0.11)$ & $(0.11)$ & $(0.13)$ \\
\hline $\mathrm{r} 2$ & 0.702 & 0.784 & 0.768 & 0.785 & 0.702 & 0.75 & 0.733 & 0.804 \\
\hline $\mathrm{N}$ & 11 & 11 & 11 & 11 & 11 & 11 & 11 & 11 \\
\hline \multicolumn{9}{|c|}{ Panel B. Urbano Hombre } \\
\hline \multirow[t]{2}{*}{$\mathrm{t}$} & -0.036 & -0.018 & -0.025 & -0.029 & -0.036 & -0.008 & -0.017 & -0.023 \\
\hline & $(0.03)$ & $(0.04)$ & $(0.04)$ & $0.05)$ & $(0.03)$ & $(0.03)$ & $(0.03)$ & $(0.04)$ \\
\hline \multirow[t]{2}{*}{$\mathrm{De}$} & $0.229 *$ & 0.173 & 0.196 & 0.197 & $0.229 *$ & 0.158 & 0.181 & 0.177 \\
\hline & $(0.10)$ & $(0.13)$ & $(0.11)$ & $0.11)$ & $(0.10)$ & $(0.09)$ & $(0.09)$ & $(0.09)$ \\
\hline \multirow[t]{2}{*}{$\mathrm{D}$} & & -0.153 & & -0.607 & & -0.265 & & $-2.856^{* * *}$ \\
\hline & & $(0.28)$ & & $1.17)$ & & $(0.22)$ & & $(0.23)$ \\
\hline \multirow[t]{2}{*}{$\mathrm{Dt}$} & & & -0.011 & 0.062 & & & -0.02 & $0.297^{* * *}$ \\
\hline & & & $(0.03)$ & $0.13)$ & & & $(0.03)$ & $(0.04)$ \\
\hline \multirow[t]{2}{*}{ cons } & $-3.466 * * *$ & $-3.494 * * *$ & $-3.485^{* * *}$ & $-3.467 * * *$ & $-3.466^{* * *}$ & $-3.522 * * *$ & $-3.505^{* * *}$ & $-3.476 * * *$ \\
\hline & $(0.18)$ & $(0.20)$ & $(0.20)$ & $0.23)$ & $(0.18)$ & $(0.19)$ & $(0.20)$ & $(0.22)$ \\
\hline $\mathrm{r} 2$ & 0.334 & 0.351 & 0.339 & 0.371 & 0.334 & 0.405 & 0.365 & 0.63 \\
\hline $\mathrm{N}$ & 11 & 11 & 11 & 11 & 11 & 11 & 11 & 11 \\
\hline \multicolumn{9}{|c|}{ Panel C. Urbano Muje } \\
\hline \multirow[t]{2}{*}{$\mathrm{t}$} & $-0.047 * *$ & -0.013 & -0.012 & -0.014 & $-0.047 * *$ & -0.032 & -0.034 & -0.036 \\
\hline & $(0.01)$ & $(0.02)$ & $(0.02)$ & $(0.03)$ & $(0.01)$ & $(0.02)$ & $(0.02)$ & $(0.02)$ \\
\hline \multirow[t]{2}{*}{ De } & $0.174 * *$ & 0.072 & 0.074 & 0.074 & $0.174 * *$ & $0.135^{*}$ & $0.142 *$ & $0.141 *$ \\
\hline & $(0.05)$ & $(0.06)$ & $(0.07)$ & $(0.07)$ & $(0.05)$ & $(0.06)$ & $(0.05)$ & $(0.06)$ \\
\hline \multirow[t]{2}{*}{$\mathrm{D}$} & & -0.277 & & -0.314 & & -0.145 & & $-0.857^{*}$ \\
\hline & & $(0.13)$ & & $(0.32)$ & & $(0.13)$ & & $(0.26)$ \\
\hline \multirow[t]{2}{*}{$\mathrm{Dt}$} & & & -0.032 & 0.005 & & & -0.014 & 0.082 \\
\hline & & & $(0.02)$ & $(0.04)$ & & & $(0.02)$ & 0.08 \\
\hline \multirow[t]{2}{*}{ cons } & $-1.758^{* * *}$ & $-1.811 * * *$ & $-1.818^{* * *}$ & $-1.808^{* * *}$ & $-1.758^{* * *}$ & $-1.789^{* * *}$ & $-1.785^{* * *}$ & $-1.777 * * *$ \\
\hline & $(0.10)$ & $(0.09)$ & $(0.09)$ & $(0.10)$ & $(0.10)$ & $(0.10)$ & $(0.10)$ & $(0.12)$ \\
\hline $\mathrm{r} 2$ & 0.74 & 0.838 & 0.823 & 0.838 & 0.74 & 0.778 & 0.765 & 0.808 \\
\hline $\mathrm{N}$ & 11 & 11 & 11 & 11 & 11 & 11 & 11 & 11 \\
\hline \multicolumn{9}{|c|}{ Panel D. Area Rural } \\
\hline $\mathrm{t}$ & $-0.069 * * *$ & $-0.081 * * *$ & $-0.081^{* * *}$ & $-0.080^{* *}$ & $-0.069^{* * *}$ & $-0.082 * * *$ & $-0.080^{* * * *}$ & $-0.079^{* * *}$ \\
\hline
\end{tabular}




\begin{tabular}{|c|c|c|c|c|c|c|c|c|}
\hline & $(0.01)$ & $(0.01)$ & $(0.02)$ & $(0.02)$ & $(0.01)$ & $(0.01)$ & $(0.01)$ & $(0.01)$ \\
\hline \multirow[t]{2}{*}{ De } & 0.044 & 0.077 & 0.077 & 0.077 & 0.044 & 0.077 & 0.071 & 0.072 \\
\hline & $(0.04)$ & $(0.04)$ & $(0.04)$ & $(0.05)$ & $(0.04)$ & $(0.04)$ & $(0.04)$ & $(0.04)$ \\
\hline \multirow[t]{2}{*}{$\mathrm{D}$} & & 0.09 & & 0.1 & & 0.122 & & $0.790 * * *$ \\
\hline & & $(0.09)$ & & $(0.36)$ & & $(0.06)$ & & $(0.07)$ \\
\hline \multirow[t]{2}{*}{$\mathrm{Dt}$} & & & 0.011 & -0.001 & & & 0.011 & $-0.077 * * *$ \\
\hline & & & $(0.01)$ & $(0.04)$ & & & $(0.01)$ & $(0.01)$ \\
\hline \multirow[t]{2}{*}{ cons } & $-0.409 * * *$ & $-0.392 * * *$ & $-0.390^{* * *}$ & $-0.393 * *$ & $-0.409 * * *$ & $-0.384^{* * * *}$ & $-0.387^{* * * *}$ & $-0.395^{* * *}$ \\
\hline & $(0.05)$ & $(0.06)$ & $(0.06)$ & $(0.07)$ & $(0.05)$ & $(0.06)$ & $(0.06)$ & $(0.07)$ \\
\hline $\mathrm{r} 2$ & 0.927 & 0.934 & 0.933 & 0.934 & 0.927 & 0.946 & 0.939 & 0.965 \\
\hline $\mathrm{N}$ & 11 & 11 & 11 & 11 & 11 & 11 & 11 & 11 \\
\hline \multicolumn{9}{|c|}{ Panel E. Rural Hombre } \\
\hline \multirow[t]{2}{*}{$\mathrm{t}$} & $-0.066 * * *$ & $-0.088 * * *$ & $-0.090^{* * *}$ & $-0.089 * * *$ & $-0.066^{* * *}$ & $-0.083^{* * *}$ & $-0.081^{* * *}$ & $-0.079 * *$ \\
\hline & $(0.01)$ & $(0.01)$ & $(0.01)$ & $(0.01)$ & $(0.01)$ & $(0.01)$ & $(0.01)$ & $(0.01)$ \\
\hline \multirow[t]{2}{*}{$\mathrm{De}$} & 0.072 & 0.136 & 0.14 & 0.139 & 0.072 & 0.115 & 0.109 & 0.11 \\
\hline & $(0.07)$ & $(0.08)$ & $(0.08)$ & $(0.08)$ & $(0.07)$ & $(0.07)$ & $(0.07)$ & $(0.08)$ \\
\hline \multirow[t]{2}{*}{$\mathrm{D}$} & & 0.174 & & 0.116 & & 0.16 & & 0.812 \\
\hline & & $(0.08)$ & & $(0.44)$ & & $(0.08)$ & & $(0.43)$ \\
\hline \multirow[t]{2}{*}{$\mathrm{Dt}$} & & & 0.022 & 0.008 & & & 0.015 & -0.075 \\
\hline & & & $(0.01)$ & $(0.05)$ & & & $(0.01)$ & $(0.05)$ \\
\hline \multirow[t]{2}{*}{ cons } & $-1.269 * * *$ & $-1.236 * * *$ & $-1.229 * * *$ & $-1.232 * * *$ & $-1.269 * * *$ & $-1.235^{* * *}$ & $-1.238^{* * *}$ & $-1.246^{* * *}$ \\
\hline & $(0.07)$ & $(0.07)$ & $(0.07)$ & $(0.07)$ & $(0.07)$ & $(0.07)$ & $(0.07)$ & $(0.08)$ \\
\hline $\mathrm{r} 2$ & 0.84 & 0.867 & 0.866 & 0.868 & 0.84 & 0.872 & 0.863 & 0.89 \\
\hline $\mathrm{N}$ & 11 & 11 & 11 & 11 & 11 & 11 & 11 & 11 \\
\hline \multicolumn{9}{|c|}{ Panel F. Rural Mujer } \\
\hline \multirow[t]{2}{*}{$\mathrm{t}$} & $-0.086^{* * *}$ & $-0.092^{* *}$ & $-0.093^{* *}$ & $-0.093 * *$ & $-0.086^{* * *}$ & $-0.099 * * *$ & $-0.097^{* * * *}$ & $-0.096 * * *$ \\
\hline & $(0.01)$ & $(0.02)$ & $(0.02)$ & $(0.02)$ & $(0.01)$ & $(0.01)$ & $(0.01)$ & $(0.02)$ \\
\hline \multirow[t]{2}{*}{$\mathrm{De}$} & 0.058 & $0.077^{*}$ & $0.079 *$ & $0.079 *$ & 0.058 & $0.092 *$ & $0.086^{*}$ & $0.087 *$ \\
\hline & $(0.03)$ & $(0.03)$ & $(0.03)$ & $(0.03)$ & $(0.03)$ & $(0.03)$ & $(0.03)$ & $(0.03)$ \\
\hline \multirow[t]{2}{*}{ D } & & 0.054 & & 0.018 & & 0.126 & & $0.738^{*}$ \\
\hline & & $(0.11)$ & & $(0.37)$ & & $(0.08)$ & & $(0.23)$ \\
\hline \multirow[t]{2}{*}{$\mathrm{Dt}$} & & & 0.007 & 0.005 & & & 0.012 & $-0.070^{*}$ \\
\hline & & & $(0.01)$ & $(0.04)$ & & & $(0.01)$ & $(0.03)$ \\
\hline \multirow[t]{2}{*}{ cons } & $0.284^{* *}$ & $0.294 * *$ & $0.297 * *$ & $0.296^{*}$ & $0.284 * *$ & $0.311^{* *}$ & $0.307 * *$ & $0.300^{*}$ \\
\hline & $(0.07)$ & $(0.08)$ & $(0.08)$ & $(0.09)$ & $(0.07)$ & $(0.07)$ & $(0.07)$ & $(0.08)$ \\
\hline r2 & 0.94 & 0.942 & 0.942 & 0.942 & 0.94 & 0.954 & 0.949 & 0.964 \\
\hline $\mathrm{N}$ & 11 & 11 & 11 & 11 & 11 & 11 & 11 & 11 \\
\hline
\end{tabular}


Tabla 19: Media de Personas con Respuestas Correctas y Diferencia Normalizada por Outcome según Sexo, Años de Educación y Grupo

\begin{tabular}{|c|c|c|c|c|c|c|}
\hline & \multicolumn{6}{|c|}{ Panel A. Muestra Completa } \\
\hline & \multicolumn{6}{|c|}{ Bolivia } \\
\hline & & tra Tratado & & & ntrol= & \\
\hline & Media & d.e. & Media & & & \\
\hline Lectura 1 & & 0.62 & 0.49 & 0.61 & 0.49 & 0.31 \\
\hline Lectura 2 & & 0.24 & 0.43 & 0.26 & 0.44 & -0.58 \\
\hline Lectura 3 & & 0.15 & 0.35 & 0.16 & 0.37 & -0.54 \\
\hline Escritura 1 & & 0.46 & 0.5 & 0.39 & 0.49 & 2.06 \\
\hline Escritura 2 & & 0.27 & 0.45 & 0.2 & 0.4 & 2.28 \\
\hline Escritura 3 & & 0.24 & 0.43 & 0.18 & 0.38 & 2.04 \\
\hline & & & Urbano & & & \\
\hline & & tra Tratado & & $\mathbf{M}$ & ntrol & \\
\hline & Media & d.e. & Media & & & \\
\hline Lectura 1 & & 0.67 & 0.47 & 0.64 & 0.48 & 0.59 \\
\hline Lectura 2 & & 0.28 & 0.45 & 0.23 & 0.42 & 1.03 \\
\hline Lectura 3 & & 0.13 & 0.34 & 0.14 & 0.35 & -0.17 \\
\hline Escritura 1 & & 0.48 & 0.5 & 0.41 & 0.49 & 1.35 \\
\hline Escritura 2 & & 0.16 & 0.37 & 0.15 & 0.35 & 0.34 \\
\hline Escritura 3 & & 0.14 & 0.35 & 0.13 & 0.33 & 0.43 \\
\hline & & & Rural & & & \\
\hline & & tra Tratado & & & ntrol & \\
\hline & Media & d.e. & Media & & & \\
\hline Lectura 1 & & 0.58 & 0.49 & 0.58 & 0.49 & 0.04 \\
\hline Lectura 2 & & 0.21 & 0.41 & 0.29 & 0.45 & -1.8 \\
\hline Lectura 3 & & 0.16 & 0.36 & 0.18 & 0.38 & -0.66 \\
\hline Escritura 1 & & 0.44 & 0.5 & 0.37 & 0.48 & 1.63 \\
\hline Escritura 2 & & 0.36 & 0.48 & 0.25 & 0.44 & 2.29 \\
\hline Escritura 3 & & 0.31 & 0.46 & 0.22 & 0.42 & 1.93 \\
\hline & & & B. Muestra Hon & bres & & \\
\hline & & & Bolivia & & & \\
\hline & & stra Tratado & & & ntrol & \\
\hline & Media & d.e. & Media & & & \\
\hline Lectura 1 & & 0.74 & 0.44 & 0.7 & 0.46 & 0.64 \\
\hline Lectura 2 & & 0.3 & 0.46 & 0.36 & 0.48 & -0.7 \\
\hline Lectura 3 & & 0.19 & 0.39 & 0.18 & 0.38 & 0.11 \\
\hline Escritura 1 & & 0.63 & 0.49 & 0.47 & 0.5 & 1.87 \\
\hline Escritura 2 & & 0.44 & 0.5 & 0.3 & 0.46 & 1.71 \\
\hline Escritura 3 & & 0.37 & 0.49 & 0.28 & 0.45 & 1.17 \\
\hline & & & Urbano & & & \\
\hline & & stra Tratado & & & ontrol & \\
\hline & Media & d.e. & Media & & & \\
\hline Lectura 1 & & 0.7 & 0.48 & 0.69 & 0.46 & 0.05 \\
\hline Lectura 2 & & 0.3 & 0.48 & 0.32 & 0.47 & -0.12 \\
\hline Lectura 3 & & 0.2 & 0.42 & 0.19 & 0.39 & 0.1 \\
\hline Escritura 1 & & 0.6 & 0.52 & 0.46 & 0.5 & 0.79 \\
\hline Escritura 2 & & 0.2 & 0.42 & 0.2 & 0.4 & 0.02 \\
\hline Escritura 3 & & 0.2 & 0.42 & 0.2 & 0.4 & 0.02 \\
\hline & & & Rural & & & \\
\hline & & stra Tratado & & & ntrol & \\
\hline & Media & d.e. & Media & & & \\
\hline Lectura 1 & & 0.76 & 0.44 & 0.7 & 0.46 & 0.68 \\
\hline Lectura 2 & & 0.3 & 0.47 & 0.38 & 0.49 & -0.81 \\
\hline Lectura 3 & & 0.18 & 0.39 & 0.17 & 0.38 & 0.09 \\
\hline Escritura 1 & & 0.64 & 0.49 & 0.48 & 0.5 & 1.6 \\
\hline Escritura 2 & & 0.52 & 0.51 & 0.36 & 0.48 & 1.61 \\
\hline Escritura 3 & & 0.42 & 0.5 & 0.32 & 0.47 & 1.06 \\
\hline & & & C. Muestra Mu & eres & & \\
\hline & & & Bolivia & & & \\
\hline & & tra Tratado & & & ntrol & \\
\hline & Media & d.e. & Media & & & \\
\hline Lectura 1 & & 0.59 & 0.49 & 0.56 & 0.5 & 0.91 \\
\hline Lectura 2 & & 0.23 & 0.42 & 0.21 & 0.41 & 0.68 \\
\hline Lectura 3 & & 0.14 & 0.35 & 0.15 & 0.36 & -0.39 \\
\hline Escritura 1 & & 0.43 & 0.5 & 0.34 & 0.47 & 2.27 \\
\hline Escritura 2 & & 0.24 & 0.43 & 0.15 & 0.36 & 2.83 \\
\hline Escritura 3 & & 0.21 & 0.41 & 0.12 & 0.33 & 2.9 \\
\hline & & & Urbano & & & \\
\hline & & stra Tratados & & & ntrol & \\
\hline & Media & d.e. & Media & & & \\
\hline Lectura 1 & & 0.66 & 0.47 & 0.62 & 0.49 & 0.84 \\
\hline Lectura 2 & & 0.28 & 0.45 & 0.2 & 0.4 & 1.55 \\
\hline Lectura 3 & & 0.13 & 0.33 & 0.12 & 0.33 & 0.1 \\
\hline Escritura 1 & & 0.47 & 0.5 & 0.39 & 0.49 & 1.38 \\
\hline Escritura 2 & & 0.16 & 0.36 & 0.13 & 0.33 & 0.68 \\
\hline Escritura 3 & & 0.14 & 0.35 & 0.1 & 0.3 & 0.93 \\
\hline & & & Rural & & & \\
\hline & & tra Tratado & & & ntrol & \\
\hline & Media & d.e. & Media & & & \\
\hline Lectura 1 & & 0.54 & 0.5 & 0.49 & 0.5 & 0.84 \\
\hline Lectura 2 & & 0.19 & 0.39 & 0.22 & 0.41 & -0.59 \\
\hline Lectura 3 & & 0.15 & 0.36 & 0.18 & 0.39 & -0.8 \\
\hline Escritura 1 & & 0.39 & 0.49 & 0.28 & 0.45 & 2.15 \\
\hline Escritura 2 & & 0.31 & 0.47 & 0.17 & 0.38 & 2.83 \\
\hline Escritura 3 & & 0.28 & 0.45 & 0.15 & 0.35 & 2.71 \\
\hline & & & $<2$ años de ed & cación & & \\
\hline & & & Bolivia & & & \\
\hline & & tra Tratado & & & ntrol & \\
\hline & Media & d.e. & Media & & & \\
\hline
\end{tabular}




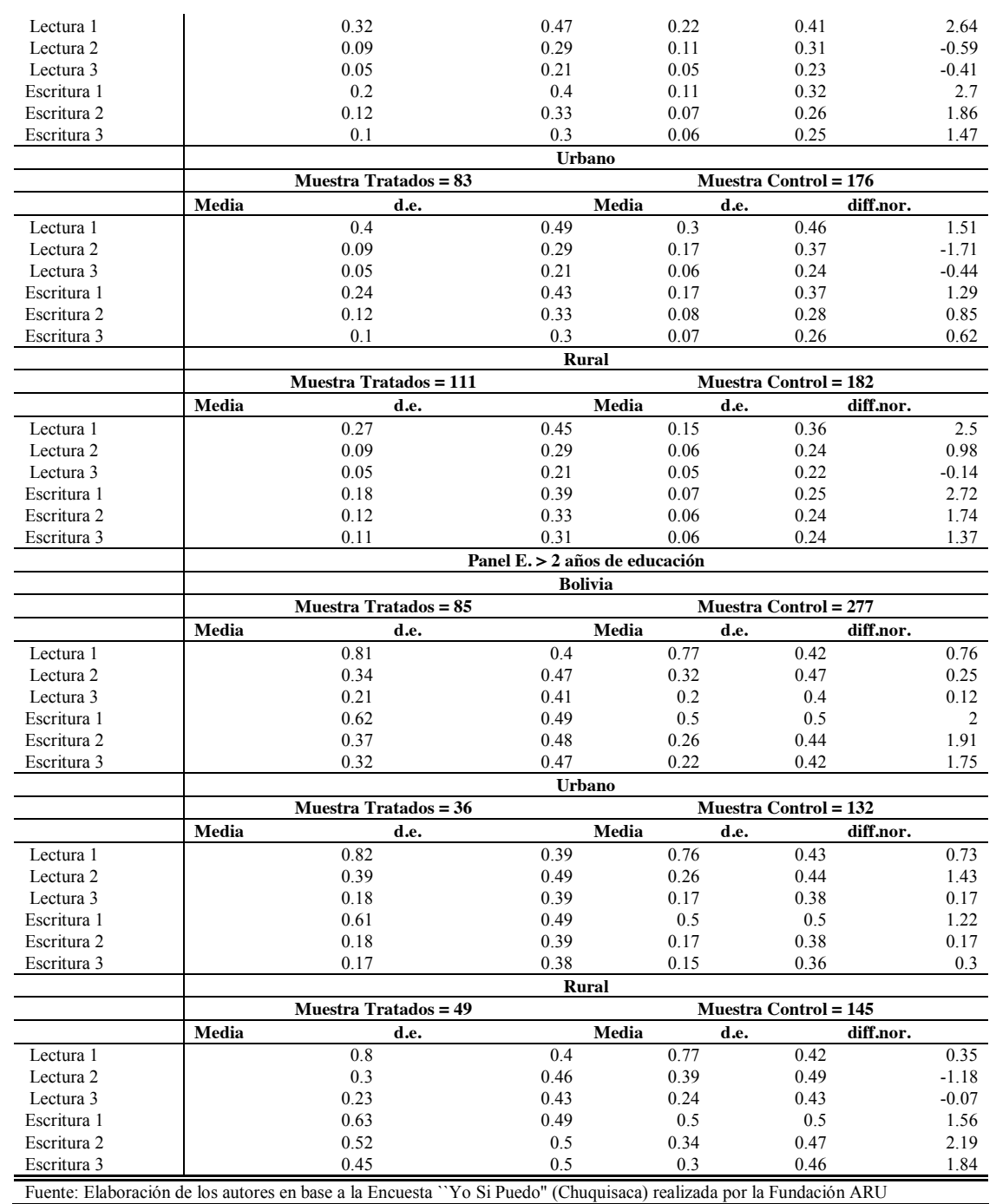


Tabla 20: Media y Diferencia Normalizada por Variable de Estudio según Muestra

\begin{tabular}{|c|c|c|c|c|c|c|c|c|}
\hline \multirow{2}{*}{ 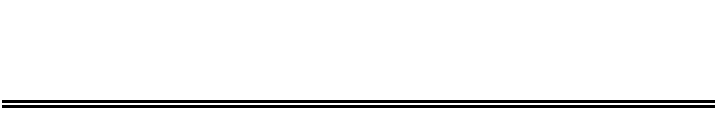 } & \multicolumn{2}{|c|}{ Muestra Tratados=279 } & \multicolumn{3}{|c|}{ Muestra Control Completa $=635$} & \multicolumn{3}{|c|}{ Muestra Control Emparejada=279 } \\
\hline & Mean & s.e. & Mean & s.e. & nor.diff. & Mean & s.e. & nor.diff. \\
\hline & 0.62 & 0.49 & 0.61 & 0.49 & 0.35 & 0.52 & 0.50 & 2.94 \\
\hline Lectura Comprensiva & 0.24 & 0.43 & 0.26 & 0.44 & -0.57 & 0.21 & 0.41 & 1.18 \\
\hline Expresión Escrita Básica & 0.46 & 0.50 & 0.39 & 0.49 & 2.05 & 0.26 & 0.44 & 5.80 \\
\hline Expresión Escrita Intermedia & 0.27 & 0.45 & 0.20 & 0.40 & 2.27 & 0.14 & 0.34 & 4.54 \\
\hline Expresión Escrita Avanzada & 0.24 & 0.43 & 0.18 & 0.38 & 2.03 & 0.12 & 0.32 & 4.14 \\
\hline Interpretación Básica & 0.43 & 0.50 & 0.41 & 0.49 & 0.44 & 0.40 & 0.49 & 0.71 \\
\hline Interpretación Basica & 0.15 & 0.35 & 0.16 & 0.37 & -0.53 & 0.14 & 0.34 & 0.43 \\
\hline Suma & 0.46 & 0.50 & 0.40 & 0.49 & 1.56 & 0.35 & 0.48 & 3.03 \\
\hline Resta & 0.24 & 0.43 & 0.23 & 0.42 & 0.50 & 0.20 & 0.40 & 1.54 \\
\hline Multiplicación & 0.12 & 0.32 & 0.12 & 0.33 & -0.26 & 0.12 & 0.32 & 0.00 \\
\hline Alguna ayuda para escribir cartas & 0.50 & 0.50 & 0.41 & 0.49 & 2.67 & 0.54 & 0.50 & -1.00 \\
\hline Alguna ayuda para leer noticias & 0.52 & 0.50 & 0.46 & 0.50 & 1.61 & 0.48 & 0.50 & 1.00 \\
\hline Alguna ayuda para llenar formularios & 0.50 & 0.50 & 0.43 & 0.50 & 1.91 & 0.45 & 0.50 & 1.49 \\
\hline Lee periodico por lo menos una vez semana & 0.08 & 0.28 & 0.10 & 0.29 & -0.67 & 0.07 & 0.26 & 0.55 \\
\hline Revisa cuentas por lo menos una vez semana & 0.11 & 0.32 & 0.10 & 0.30 & 0.62 & 0.08 & 0.28 & 1.46 \\
\hline Edad & 44.42 & 12.57 & 40.67 & 13.58 & 4.04 & 45.43 & 12.24 & -1.13 \\
\hline Edad2 & 2130.15 & 1116.54 & 1838.48 & 1131.34 & 3.62 & 2212.90 & 1078.02 & -1.04 \\
\hline Mujer & 0.85 & 0.36 & 0.65 & 0.48 & 6.89 & 0.85 & 0.36 & 0.00 \\
\hline Años de Educación & 2.32 & 1.86 & 2.83 & 1.90 & -3.80 & 2.36 & 1.90 & -0.32 \\
\hline Casados & 0.79 & 0.41 & 0.74 & 0.44 & 1.76 & 0.79 & 0.41 & -0.12 \\
\hline Casados*Urbano & 0.33 & 0.47 & 0.40 & 0.49 & -2.08 & 0.37 & 0.48 & -1.15 \\
\hline Certificado de Nacimiento & 0.68 & 0.47 & 0.61 & 0.49 & 1.95 & 0.68 & 0.47 & -0.21 \\
\hline Nunca recibe ayuda para escribir cartas & 0.39 & 0.49 & 0.55 & 0.50 & -4.39 & 0.34 & 0.48 & 1.44 \\
\hline Cálculos matemáticos en última ocup. & 0.25 & 0.43 & 0.20 & 0.40 & 1.72 & 0.23 & 0.42 & 0.69 \\
\hline Lectura direcciones primera ocup. & 0.27 & 0.44 & 0.45 & 0.50 & -5.46 & 0.29 & 0.45 & -0.67 \\
\hline
\end{tabular}


Tabla 21. Resultados de Estimaciones según Muestra

\begin{tabular}{|c|c|c|c|c|c|c|}
\hline & \multicolumn{3}{|c|}{ Muestra No Emparejada } & \multicolumn{3}{|c|}{ Muestra Emparejada } \\
\hline & Logit & Efectos Marginales & $\mathbf{X +}$ & Logit & Efect. Marginales & $\mathrm{X}+$ \\
\hline \multirow[t]{2}{*}{ Edad } & $0.082 * *$ & $0.016^{* *}$ & 41.82 & -0.009 & -0.002 & 44.22 \\
\hline & $(0.039)$ & $(0.008)$ & & $(0.047)$ & $(0.001)$ & \\
\hline \multirow[t]{2}{*}{ Edad $^{\wedge} 2$} & $-0.001 *$ & $-0.000 *$ & 1927.52 & 0.000 & 0.000 & 2110.75 \\
\hline & $(0.000)$ & $(0.000)$ & & $(0.001)$ & $(0.000)$ & \\
\hline \multirow[t]{2}{*}{ Mujer } & $1.259 * * *$ & $0.220 * * *$ & 0.71 & 0.013 & 0.003 & 0.85 \\
\hline & $(0.202)$ & $(0.030)$ & & $(0.244)$ & $(0.061)$ & \\
\hline \multirow[t]{2}{*}{ Años de Educacion } & $-0.096 * *$ & $-0.019 * *$ & 2.68 & -0.031 & -0.008 & 2.38 \\
\hline & $(0.046)$ & $(0.009)$ & & $(0.050)$ & $(0.012)$ & \\
\hline \multirow[t]{2}{*}{ Casado } & $0.560 * * *$ & $0.105 * * *$ & 0.75 & 0.087 & 0.022 & 0.79 \\
\hline & $(0.208)$ & $(0.036)$ & & $(0.231)$ & $(0.058)$ & \\
\hline \multirow[t]{2}{*}{ Casado*Urbano } & $-0.497 * * *$ & $-0.097 * * *$ & 0.38 & -0.229 & -0.057 & 0.35 \\
\hline & $(0.192)$ & $(0.036)$ & & $(0.216)$ & $(0.053)$ & \\
\hline \multirow[t]{2}{*}{ Certificado de Nacimiento } & $0.369^{* *}$ & $0.072 * *$ & 0.63 & 0.036 & 0.009 & 0.68 \\
\hline & $(0.165)$ & $(0.031)$ & & $(0.186)$ & $(0.046)$ & \\
\hline \multirow{4}{*}{$\begin{array}{l}\text { Direcciones en primera } \\
\text { ocup. }\end{array}$} & $0.658 * * *$ & $0.142 * * *$ & 0.50 & 0.045 & 0.011 & 0.25 \\
\hline & $(0.192)$ & $(0.044)$ & & $(0.208)$ & $(0.052)$ & \\
\hline & $-0.704 * * *$ & $-0.136 * * *$ & 0.21 & 0.273 & 0.068 & 0.25 \\
\hline & $(0.181)$ & $(0.033)$ & & $(0.217)$ & $(0.054)$ & \\
\hline \multirow[t]{2}{*}{ Constante } & $-3.902 * * *$ & & 0.39 & 0.099 & & \\
\hline & $(0.838)$ & & & $(1.016)$ & & \\
\hline Observations & 914 & & & 558 & & \\
\hline R-squared & 0.10 & & & 0.00 & & \\
\hline
\end{tabular}

Fuente: Elaboración de los autores en base a la Encuesta "Yo Si Puedo" (Chuquisaca) realizada por la Fundación ARU Errores estándar en paréntisis

* significativo al 10\%; ** significativo al 5\%; *** significativo al $1 \%$

+ Valor de variable utilizada para el cálculo de efectos marginales 
Tabla 22: Efecto de Tratamiento Medio para la Muestra del PNA-Bolivia por Tipo de Muestra y Especificación según Indicador de Resultado

\begin{tabular}{|c|c|c|c|c|c|c|c|c|c|c|c|c|c|c|c|c|c|c|}
\hline & \multicolumn{3}{|c|}{$\begin{array}{c}\text { Panel A } \\
\text { Lectura } 1\end{array}$} & \multicolumn{3}{|c|}{$\begin{array}{c}\text { Panel B } \\
\text { Lectura } 2 \\
\end{array}$} & \multicolumn{3}{|c|}{$\begin{array}{c}\text { Panel C } \\
\text { Lectura } 3 \\
\end{array}$} & \multicolumn{3}{|c|}{$\begin{array}{c}\text { Panel E } \\
\text { Escritura 1 }\end{array}$} & \multicolumn{3}{|c|}{$\begin{array}{c}\text { Panel F } \\
\text { Escritura 2 }\end{array}$} & \multicolumn{3}{|c|}{$\begin{array}{c}\text { Panel G } \\
\text { Escritura } 3 \\
\end{array}$} \\
\hline & \multicolumn{18}{|c|}{ Muestra No Emparejada con Un Set Extendido de Variables } \\
\hline & SATT & d.e. & $\mathbf{t}$ & SATT & d.e. & $\mathbf{t}$ & SATT & d.e. & $\mathbf{t}$ & SATT & d.e. & t & SATT & d.e. & $\mathbf{t}$ & SATT & d.e. & $\mathbf{t}$ \\
\hline Diferencias Simples & 0.012 & 0.035 & 0.349 & -0.018 & 0.031 & -0.569 & -0.014 & 0.026 & -0.531 & 0.073 & 0.036 & 2.050 & 0.071 & 0.031 & 2.278 & 0.060 & 0.030 & 2.030 \\
\hline Regresión (Separado) & 0.081 & 0.030 & 2.697 & 0.017 & 0.030 & 0.561 & 0.062 & 0.029 & 2.149 & 0.000 & 0.026 & 0.016 & 0.105 & 0.033 & 3.196 & 0.073 & 0.030 & 2.434 \\
\hline Ponderación & 0.089 & 0.038 & 2.358 & 0.019 & 0.033 & 0.565 & -0.003 & 0.028 & -0.089 & 0.108 & 0.039 & 2.801 & 0.083 & 0.034 & 2.468 & 0.071 & 0.032 & 2.194 \\
\hline Estratificación & 0.101 & 0.037 & 2.707 & 0.026 & 0.032 & 0.796 & 0.002 & 0.028 & 0.063 & 0.132 & 0.038 & 3.492 & 0.101 & 0.033 & 3.084 & 0.087 & 0.031 & 2.779 \\
\hline Emparejamiento & 0.091 & 0.033 & 2.741 & 0.037 & 0.033 & 1.129 & 0.006 & 0.029 & 0.203 & 0.123 & 0.036 & 3.463 & 0.104 & 0.031 & 3.325 & 0.083 & 0.030 & 2.759 \\
\hline Ponderación y Regresión & 0.096 & 0.031 & 3.120 & 0.021 & 0.030 & 0.698 & -0.001 & 0.027 & -0.039 & 0.114 & 0.033 & 3.442 & 0.086 & 0.031 & 2.819 & 0.073 & 0.030 & 2.476 \\
\hline $\begin{array}{l}\text { Estratificación y Regresión } \\
\text { Emparejamiento y }\end{array}$ & 0.100 & 0.031 & 3.242 & 0.024 & 0.029 & 0.827 & -0.002 & 0.027 & -0.056 & 0.128 & 0.033 & 3.837 & 0.102 & 0.030 & 3.392 & 0.086 & 0.029 & 2.950 \\
\hline \multirow[t]{3}{*}{ Regresión } & 0.104 & 0.033 & 3.165 & 0.037 & 0.032 & 1.129 & 0.005 & 0.028 & 0.180 & 0.129 & 0.036 & 3.638 & 0.104 & 0.031 & 3.353 & 0.083 & 0.030 & 2.746 \\
\hline & \multicolumn{18}{|c|}{ Muestra Emparejada con Un Set Extendido de Variables } \\
\hline & SATT & d.e. & t & SATT & d.e. & $\mathbf{t}$ & SATT & d.e. & $\mathbf{t}$ & SATT & d.e. & $\mathbf{t}$ & SATT & d.e. & $\mathbf{t}$ & SATT & d.e. & $\mathbf{t}$ \\
\hline Diferencias Simples & 0.086 & 0.042 & 2.061 & 0.011 & 0.036 & 0.298 & 0.014 & 0.029 & 0.488 & 0.140 & 0.041 & 3.416 & 0.086 & 0.035 & 2.425 & 0.079 & 0.034 & 2.348 \\
\hline Regresión (Separado) & 0.101 & 0.032 & 3.184 & 0.011 & 0.033 & 0.333 & 0.004 & 0.028 & 0.130 & 0.143 & 0.035 & 4.084 & 0.084 & 0.032 & 2.634 & 0.075 & 0.031 & 2.424 \\
\hline Ponderación & 0.100 & 0.042 & 2.407 & 0.012 & 0.036 & 0.339 & 0.005 & 0.030 & 0.168 & 0.143 & 0.041 & 3.524 & 0.085 & 0.036 & 2.376 & 0.075 & 0.034 & 2.226 \\
\hline Estratificación & 0.090 & 0.041 & 2.198 & 0.006 & 0.036 & 0.173 & 0.006 & 0.030 & 0.183 & 0.138 & 0.040 & 3.426 & 0.083 & 0.035 & 2.346 & 0.074 & 0.033 & 2.211 \\
\hline Emparejamiento & 0.104 & 0.036 & 2.910 & 0.021 & 0.034 & 0.617 & 0.037 & 0.029 & 1.254 & 0.160 & 0.040 & 4.055 & 0.087 & 0.036 & 2.427 & 0.072 & 0.034 & 2.078 \\
\hline Ponderación y Regresión & 0.100 & 0.032 & 3.135 & 0.012 & 0.032 & 0.364 & 0.004 & 0.028 & 0.145 & 0.144 & 0.035 & 4.086 & 0.084 & 0.032 & 2.604 & 0.075 & 0.031 & 2.393 \\
\hline $\begin{array}{l}\text { Estratificación y Regresión } \\
\text { Emparejamiento y }\end{array}$ & 0.097 & 0.033 & 2.932 & 0.016 & 0.032 & 0.508 & 0.009 & 0.027 & 0.322 & 0.150 & 0.036 & 4.190 & 0.095 & 0.032 & 2.953 & 0.088 & 0.031 & 2.838 \\
\hline Regresión & 0.102 & 0.035 & 2.944 & 0.007 & 0.034 & 0.217 & 0.026 & 0.029 & 0.907 & 0.152 & 0.039 & 3.883 & 0.082 & 0.035 & 2.319 & 0.067 & 0.034 & 1.967 \\
\hline
\end{tabular}

Fuente: Elaboración de los autores en base a la Encuesta "Yo Si Puedo" (Chuquisaca) realizada por Fundación ARU 
Tabla 23: Efecto de Tratamiento Medio para la Muestra del PNA-Bolivia por Tipo de Muestra y Especificación según Pseudo- Indicador de Resultado

\begin{tabular}{|c|c|c|c|c|c|c|c|c|c|c|c|c|c|c|c|c|c|c|}
\hline & \multicolumn{3}{|c|}{$\begin{array}{c}\text { Panel A } \\
\text { Suma }\end{array}$} & \multicolumn{3}{|c|}{$\begin{array}{c}\text { Panel B } \\
\text { Resta } \\
\end{array}$} & \multicolumn{3}{|c|}{$\begin{array}{c}\text { Panel C } \\
\text { Multiplicación }\end{array}$} & \multicolumn{3}{|c|}{$\begin{array}{c}\text { Panel E } \\
\text { Ninguna ayuda en carta }\end{array}$} & \multicolumn{3}{|c|}{$\begin{array}{c}\text { Panel F } \\
\text { Ninguna ayuda en noticias }\end{array}$} & \multicolumn{3}{|c|}{$\begin{array}{c}\text { Panel G } \\
\text { Ninguna ayuda en formularios }\end{array}$} \\
\hline & \multicolumn{18}{|c|}{ Muestra Completa } \\
\hline & SATT & d.e. & $\mathrm{t}$ & SATT & d.e. & $\mathrm{t}$ & SATT & d.e. & $\mathrm{t}$ & SATT & d.e. & $\mathrm{t}$ & SATT & d.e. & $\mathrm{t}$ & SATT & d.e. & $\mathbf{t}$ \\
\hline Diferencias Simples & 0.056 & 0.036 & 1.559 & 0.015 & 0.031 & 0.501 & -0.006 & 0.023 & -0.262 & -0.155 & 0.035 & -4.395 & -0.123 & 0.035 & -3.491 & -0.118 & 0.035 & -3.357 \\
\hline Regresión (Separado) & 0.103 & 0.033 & 3.175 & 0.035 & 0.029 & 1.182 & 0.001 & 0.024 & 0.04 & -0.181 & 0.038 & -4.76 & -0.154 & 0.038 & -4.097 & -0.14 & 0.038 & -3.697 \\
\hline Ponderación & 0.105 & 0.038 & 2.794 & 0.033 & 0.033 & 0.975 & 0.001 & 0.025 & 0.043 & -0.175 & 0.041 & -4.281 & -0.156 & 0.041 & -3.834 & -0.138 & 0.041 & -3.385 \\
\hline Estratificación & 0.119 & 0.037 & 3.186 & 0.043 & 0.033 & 1.334 & 0.006 & 0.025 & 0.242 & -0.172 & 0.039 & -4.363 & -0.16 & 0.039 & -4.086 & -0.138 & 0.039 & -3.545 \\
\hline $\begin{array}{l}\text { Emparejamiento } \\
\text { Ponderación y }\end{array}$ & 0.105 & 0.037 & 2.838 & 0.059 & 0.033 & 1.759 & 0.018 & 0.025 & 0.694 & -0.181 & 0.042 & -4.344 & -0.155 & 0.042 & -3.72 & -0.135 & 0.041 & -3.252 \\
\hline $\begin{array}{l}\text { Regresión } \\
\text { Estratificación y }\end{array}$ & 0.111 & 0.033 & 3.344 & 0.035 & 0.03 & 1.162 & 0.003 & 0.024 & 0.135 & -0.175 & 0.039 & -4.539 & -0.155 & 0.039 & -4.015 & -0.139 & 0.039 & -3.581 \\
\hline $\begin{array}{l}\text { Regresión } \\
\text { Emparejamiento y }\end{array}$ & 0.116 & 0.034 & 3.415 & 0.035 & 0.031 & 1.132 & 0.001 & 0.024 & 0.041 & -0.164 & 0.04 & -4.083 & -0.152 & 0.04 & -3.827 & -0.131 & 0.04 & -3.291 \\
\hline \multirow[t]{3}{*}{ Regresión } & 0.111 & 0.037 & 3.053 & 0.062 & 0.033 & 1.858 & 0.02 & 0.025 & 0.801 & -0.187 & 0.042 & -4.492 & -0.162 & 0.042 & -3.89 & -0.139 & 0.041 & -3.365 \\
\hline & \multicolumn{18}{|c|}{ Muestra Emparejada } \\
\hline & SATT & d.e. & $\mathbf{t}$ & SATT & d.e. & $\mathbf{t}$ & SATT & d.e. & $\mathbf{t}$ & SATT & d.e. & $\mathbf{t}$ & SATT & d.e. & $\mathbf{t}$ & SATT & d.e. & $\mathrm{t}$ \\
\hline Diferencias Simples & 0.093 & 0.042 & 2.242 & 0.014 & 0.036 & 0.398 & -0.014 & 0.028 & -0.51 & -0.229 & 0.041 & -5.558 & -0.176 & 0.042 & -4.218 & -0.19 & 0.042 & -4.57 \\
\hline Regresión (Separado) & 0.097 & 0.035 & 2.78 & 0.012 & 0.032 & 0.369 & -0.014 & 0.027 & -0.523 & -0.235 & 0.041 & -5.775 & -0.19 & 0.04 & -4.715 & -0.191 & 0.04 & -4.723 \\
\hline Ponderación & 0.098 & 0.041 & 2.362 & 0.012 & 0.036 & 0.325 & -0.014 & 0.028 & -0.493 & -0.234 & 0.042 & -5.615 & -0.188 & 0.042 & -4.47 & -0.19 & 0.042 & -4.543 \\
\hline Estratificación & 0.1 & 0.041 & 2.453 & 0.017 & 0.036 & 0.466 & -0.011 & 0.028 & -0.412 & -0.233 & 0.041 & -5.625 & -0.186 & 0.042 & -4.48 & -0.186 & 0.042 & -4.435 \\
\hline $\begin{array}{l}\text { Emparejamiento } \\
\text { Ponderación y }\end{array}$ & 0.103 & 0.04 & 2.575 & 0.014 & 0.036 & 0.406 & -0.011 & 0.028 & -0.375 & -0.236 & 0.044 & -5.359 & -0.158 & 0.044 & -3.597 & -0.193 & 0.044 & -4.372 \\
\hline $\begin{array}{l}\text { Regresión } \\
\text { Estratificación y }\end{array}$ & 0.098 & 0.036 & 2.751 & 0.012 & 0.033 & 0.37 & -0.013 & 0.027 & -0.503 & -0.235 & 0.041 & -5.717 & -0.189 & 0.041 & -4.563 & -0.191 & 0.041 & -4.662 \\
\hline $\begin{array}{l}\text { Regresión } \\
\text { Emparejamiento y }\end{array}$ & 0.118 & 0.036 & 3.33 & 0.017 & 0.032 & 0.535 & -0.004 & 0.026 & -0.17 & -0.241 & 0.042 & -5.716 & -0.19 & 0.042 & -4.514 & -0.188 & 0.042 & -4.436 \\
\hline Regresión & 0.085 & 0.04 & 2.139 & 0.002 & 0.035 & 0.052 & -0.018 & 0.028 & -0.653 & -0.236 & 0.044 & -5.336 & -0.163 & 0.044 & -3.688 & -0.184 & 0.044 & -4.183 \\
\hline
\end{tabular}

Fuente: Elaboración de los autores en base a la Encuesta "Yo Si Puedo" (Chuquisaca) realizada por Fundación ARU 


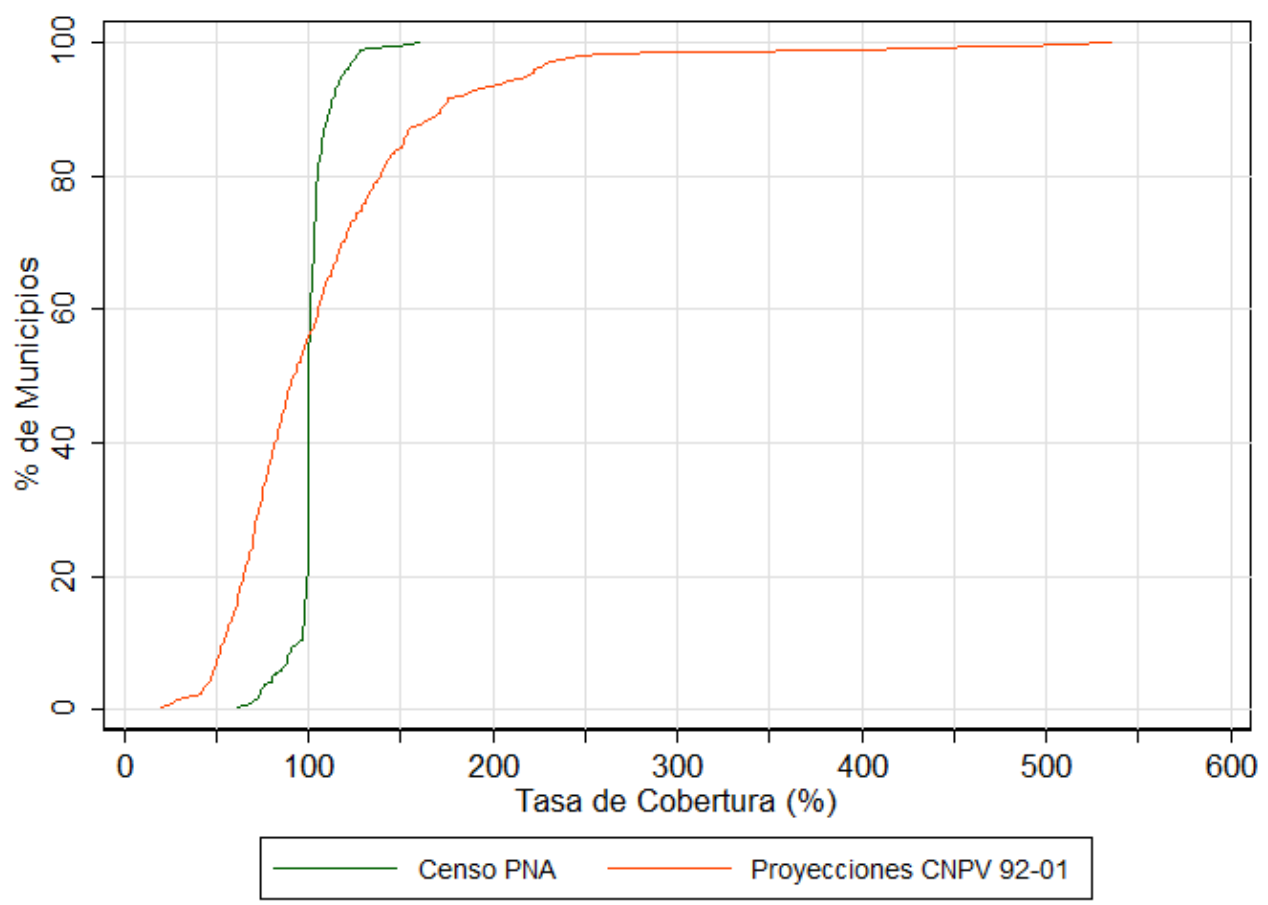

Figura 1: Distribución de Cobertura de Municipios 


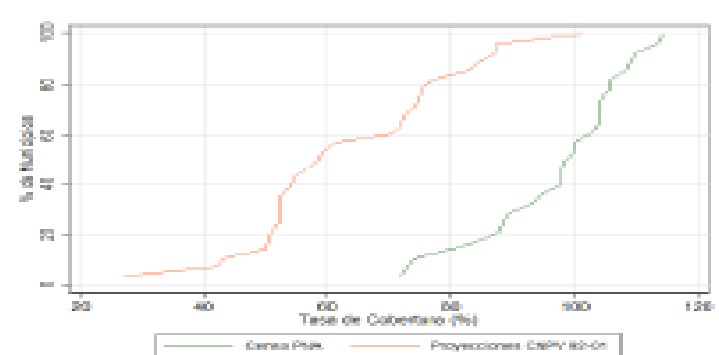

(a) Chuquisaca

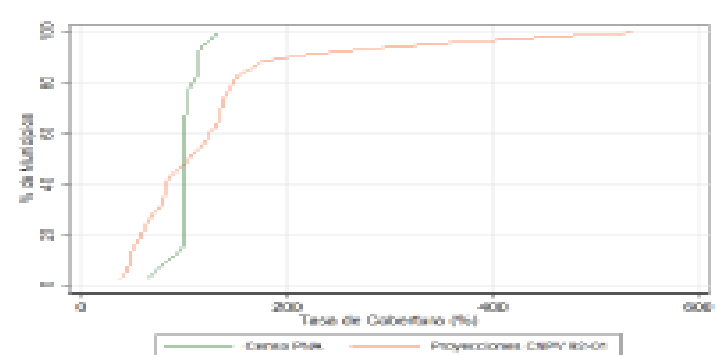

(d) Oruro

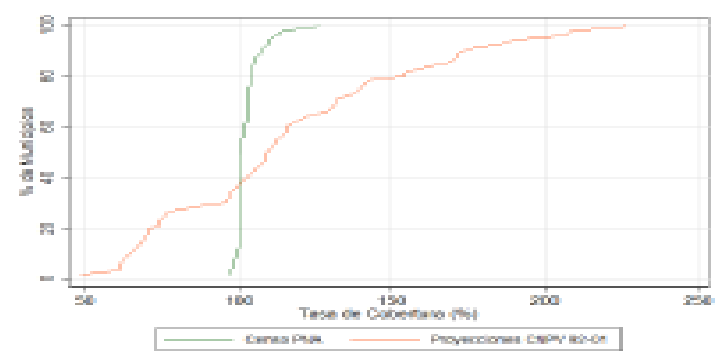

(g) Santa Cru

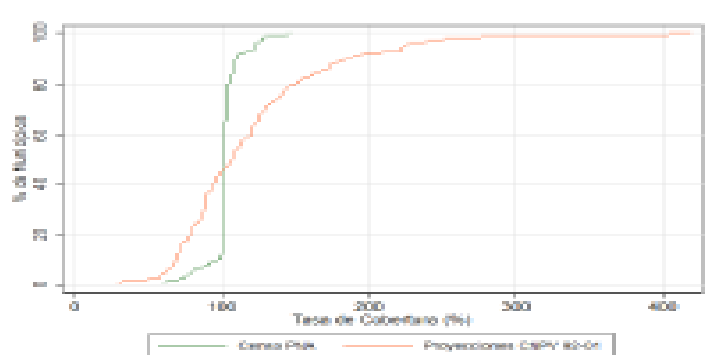

(b) La Paz

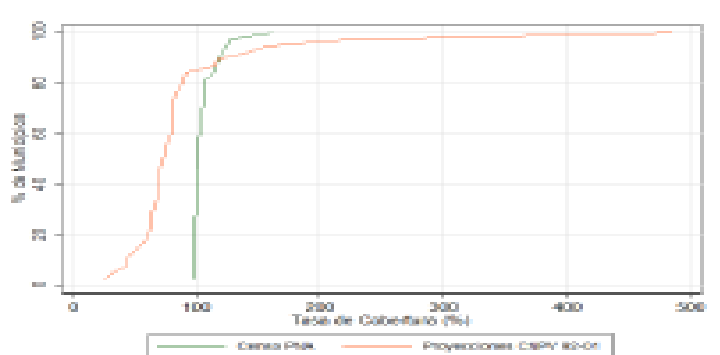

(e) Potosí

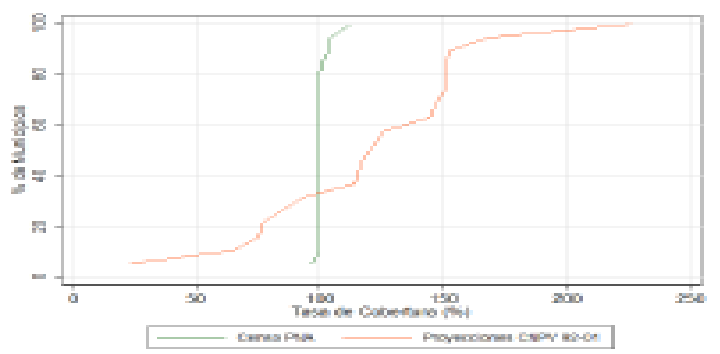

(h) Beni

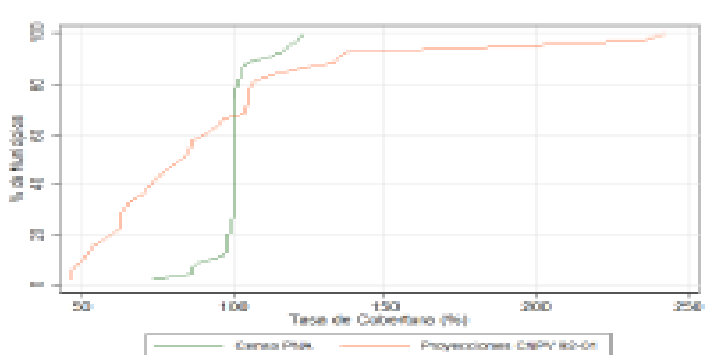

(c) Cochabamba

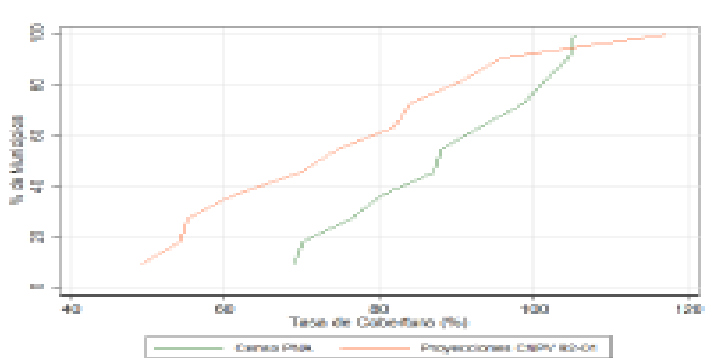

(f) Tarija

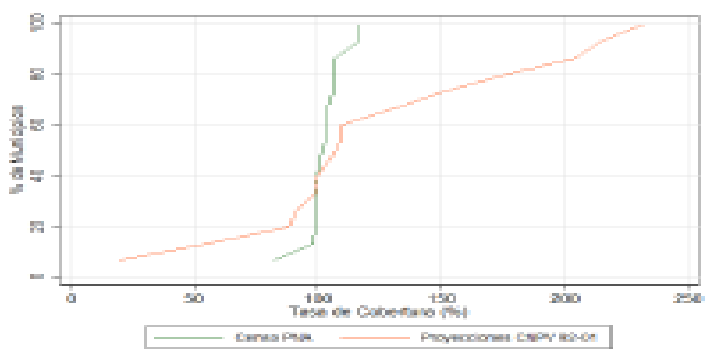

(i) Pando

Figura 2: Distribución de Cobertura de Municipios según Departamento 


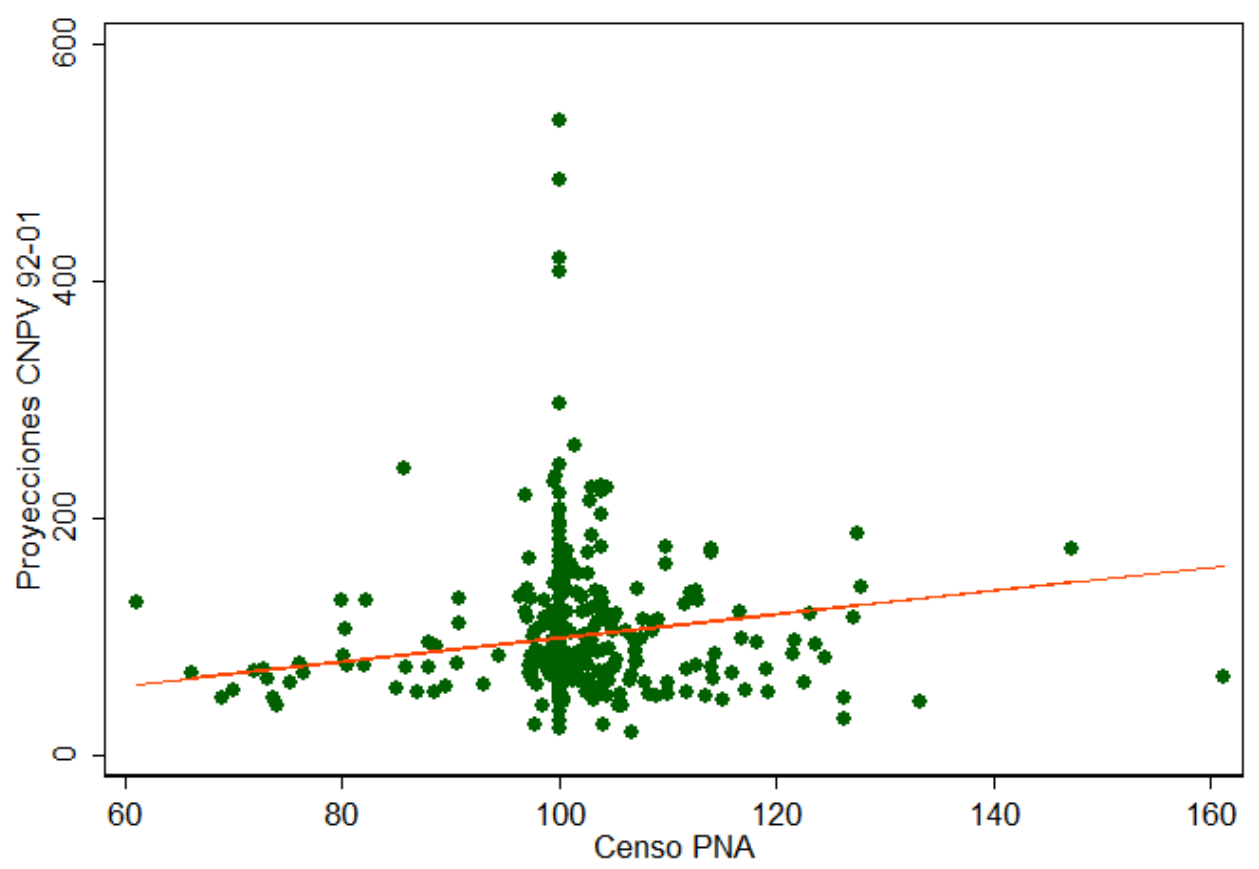

Nota: La linea continua representa asociación perfecta entre las dos fuentes

Figura 3: Asociación de Cobertura de Municipios entre Fuentes de Información 


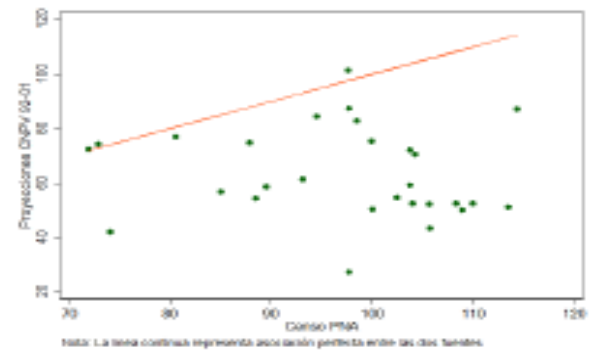

(a) Chuquisaca

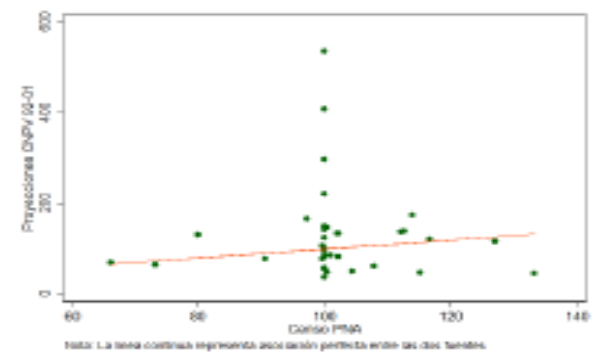

(d) Oruro

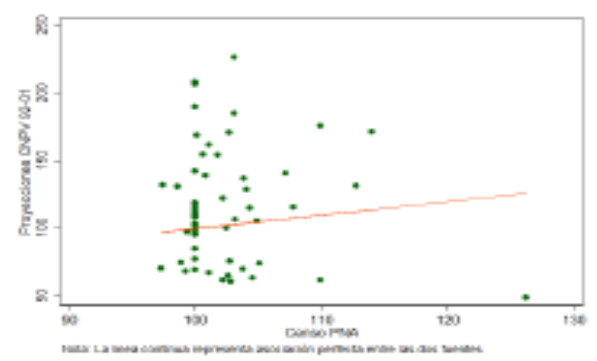

(g) Santa Cru

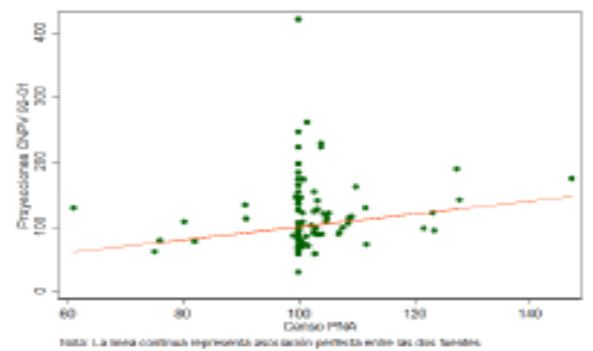

(b) La Paz

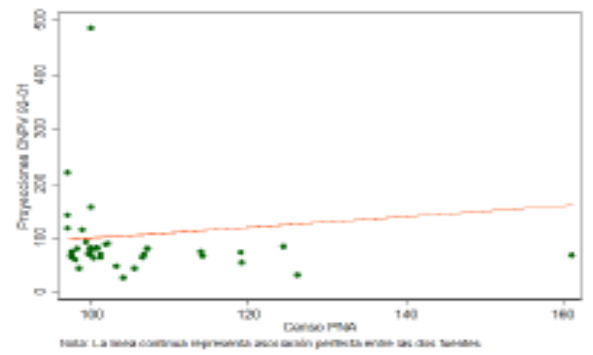

(e) Potosí

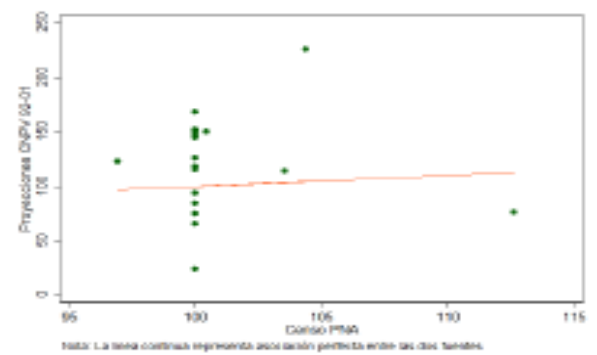

(h) Beni

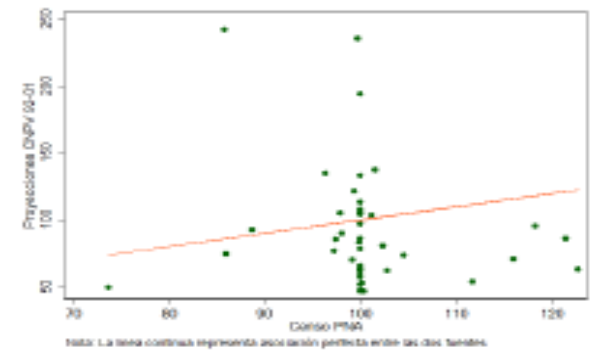

(c) Cochabamba

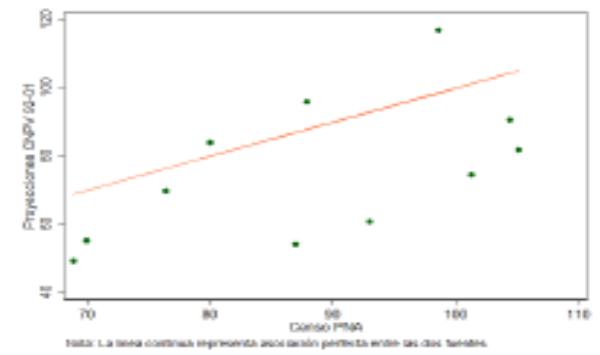

(f) Tarija

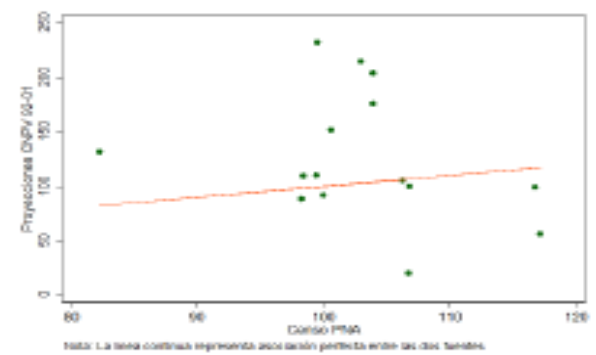

(i) Pando

Figura 4: Asociación de Cobertura de Municipios por Fuente de Información y Departamento 


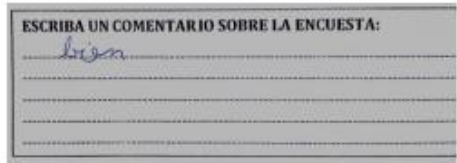

(a) Ejemplo: Escritura 1

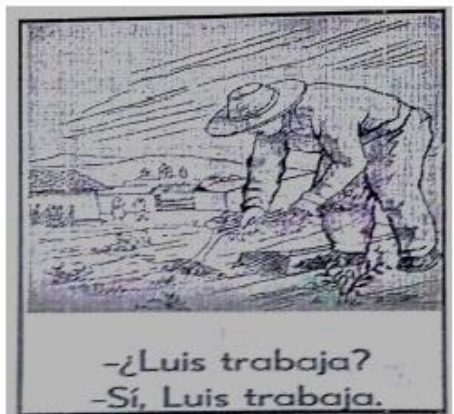

"Está Trabajando un Hombre"

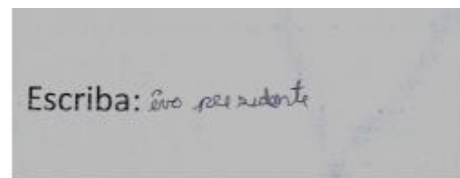

(b) Ejemplo: Escritura 2

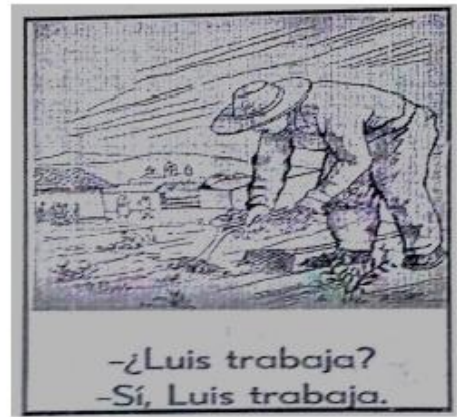

"Hombre Trabajando"

"Luis Siembra"

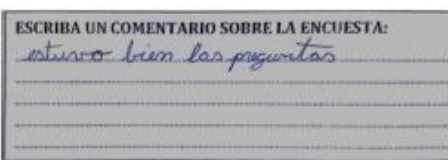

(c) Ejemplo: Escritra 3

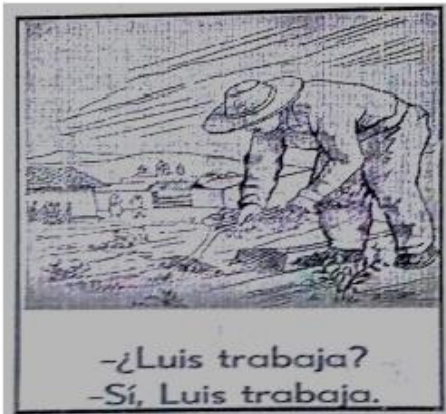

"Luis Trabaja"

Figura 5: Ejemplos de Respuesta de Boletas sobre Indicadores de Resutado de Escritura y Lectura

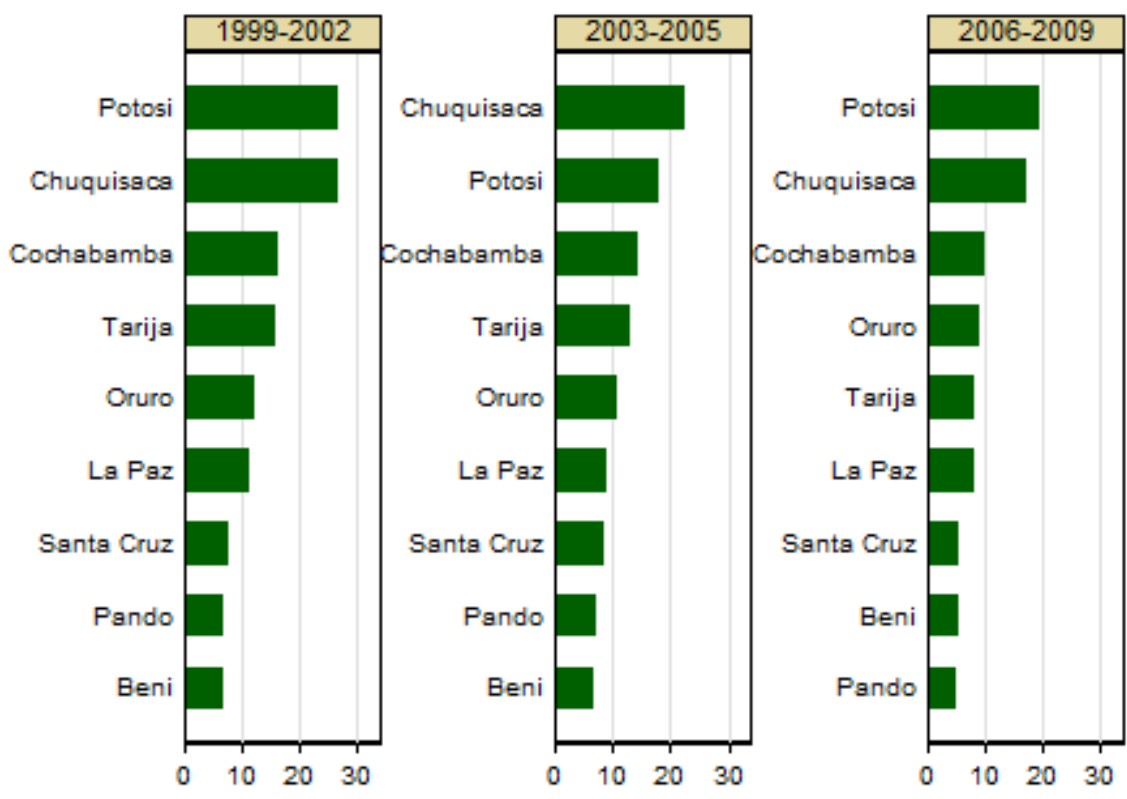

Figura 6: Tasas de Analfabetismo por Departamentos según Periodo 


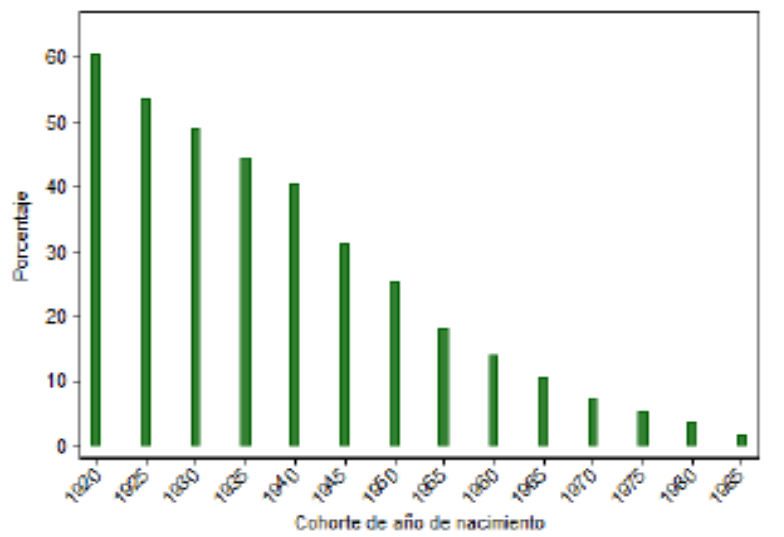

(a) Bolivia

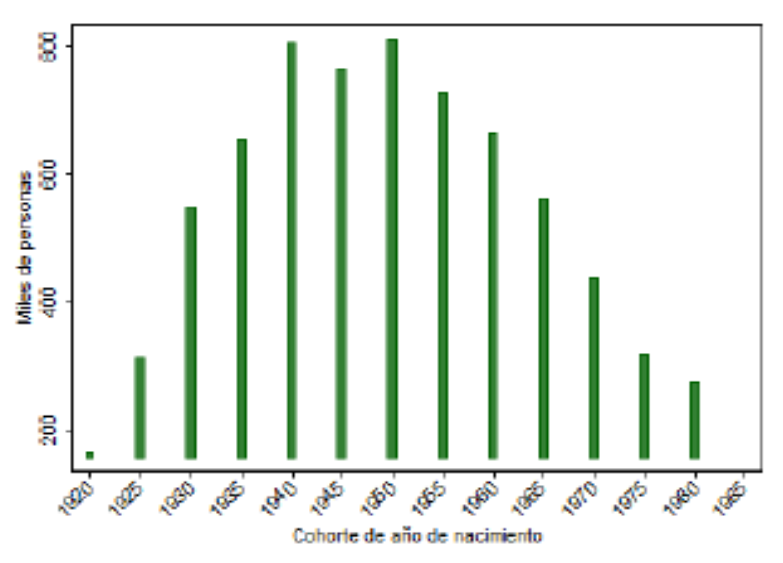

(d) Bolivia

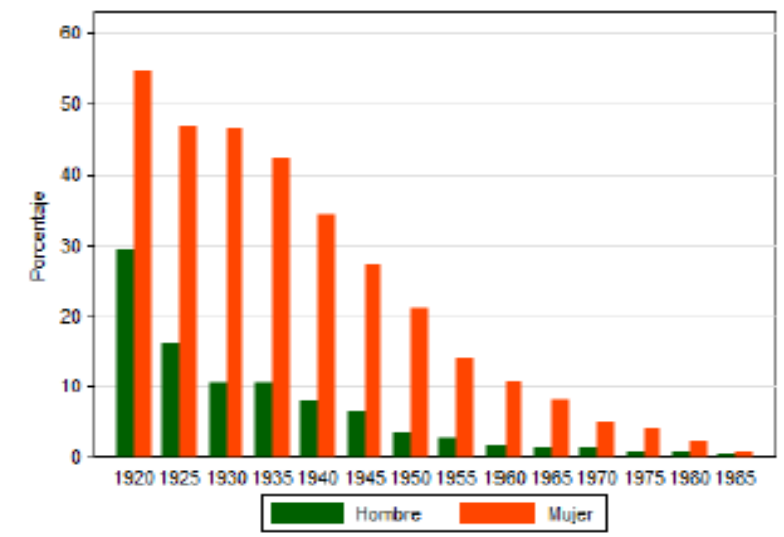

(b) Área Urbana

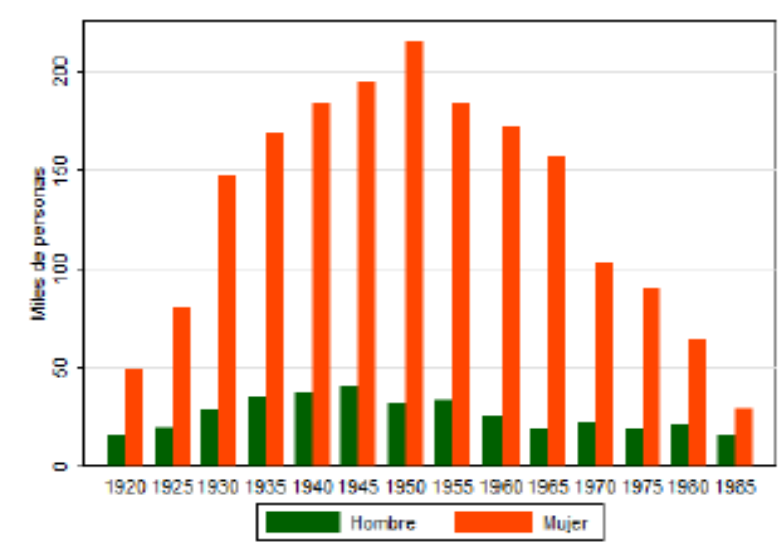

(e) Área Urbana

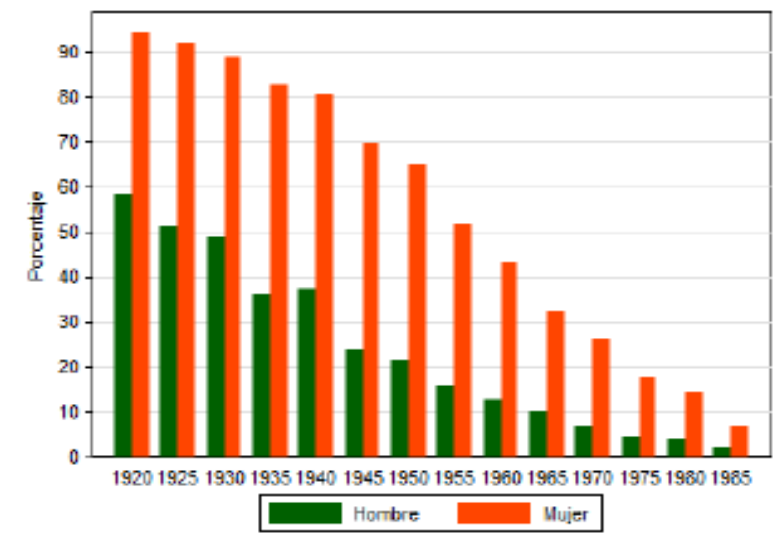

(c) Área Rural

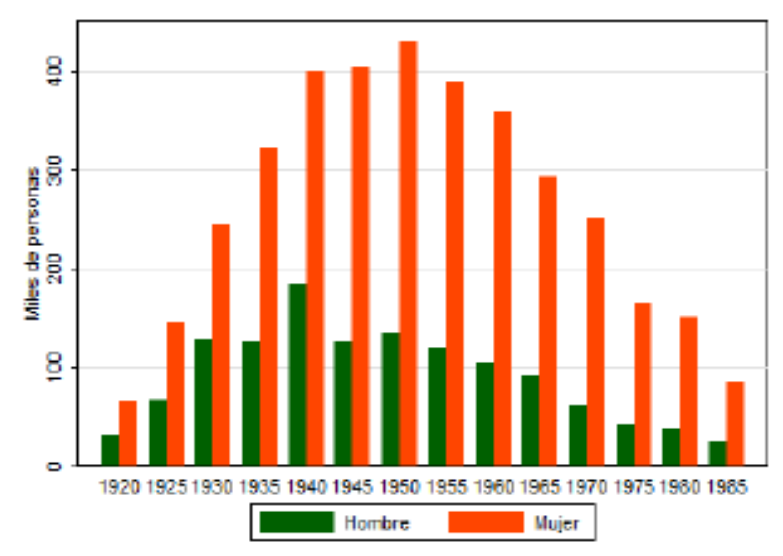

(f) Área Rural

Figura 7: Tasas de Analfabetismo y Población Analfabeta por Año de Nacimiento según Área 


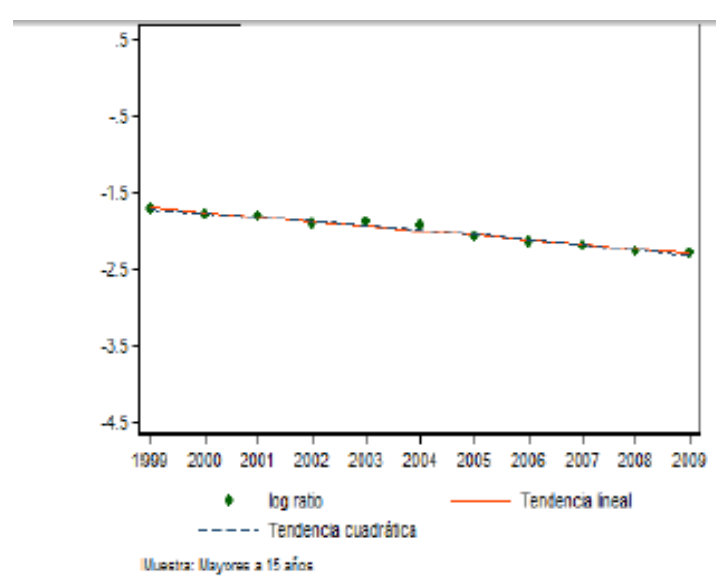

(a) Logaritmo del Ratio de analfabetismo $\left(\frac{\pi}{1-\pi}\right)$

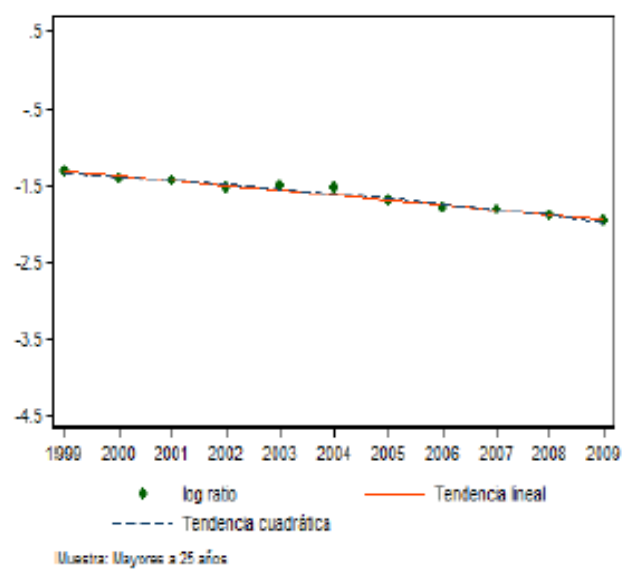

(d) Logaritmo del Ratio de analfabetismo $\left(\frac{\pi}{1-\pi}\right)$

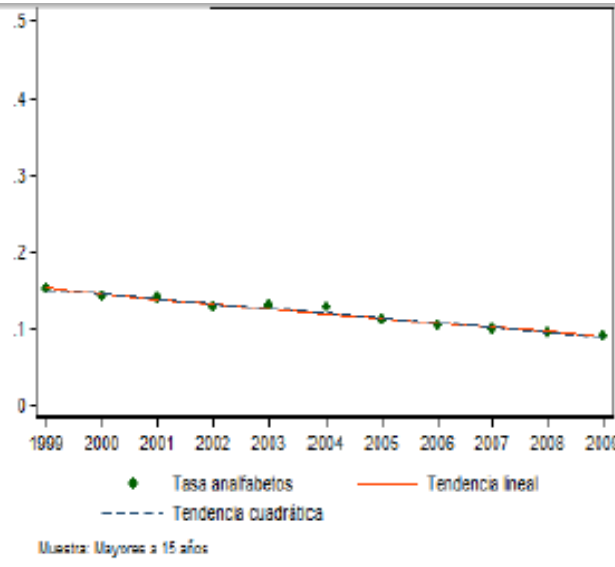

(b) Tasa de analfabetismo $(\pi)$

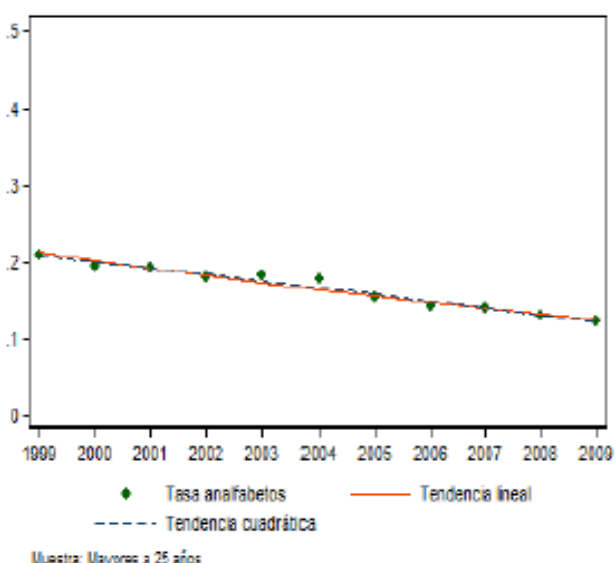

(e) Tasa de analfabetismo $(\pi)$

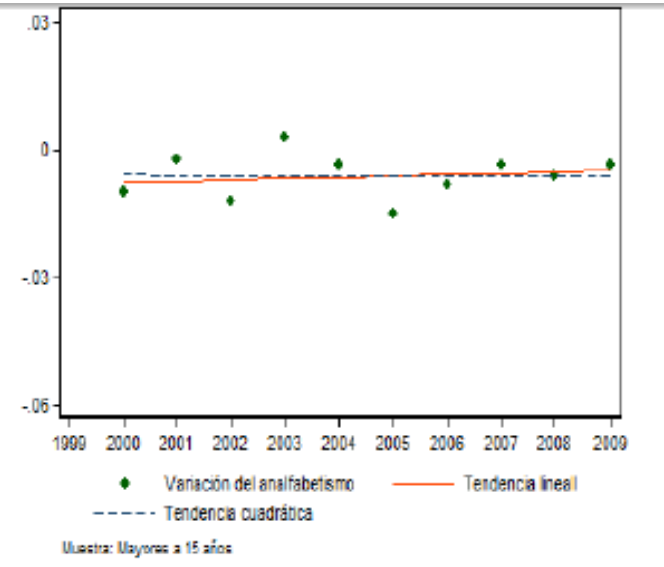

(c) Variación del analfabetismo $\left(\frac{\partial \pi}{\partial t}\right)$

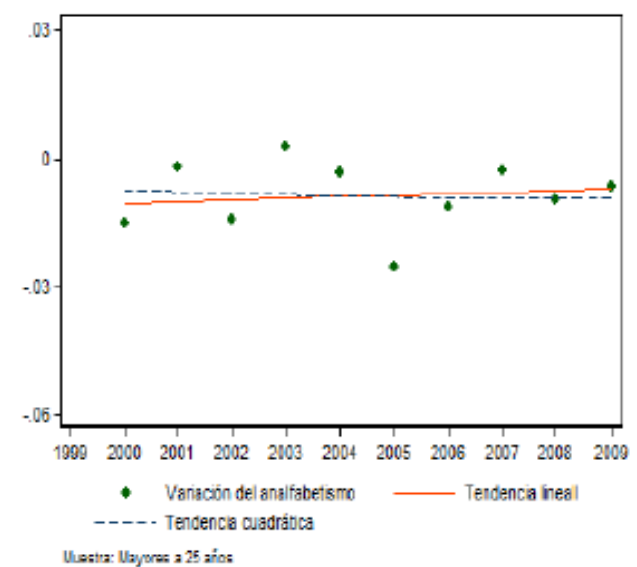

(f) Variación del analfabetismo $\left(\frac{\partial \pi}{\partial t}\right)$

Figura 8: Evaluación de Tendencias. Bolivia 


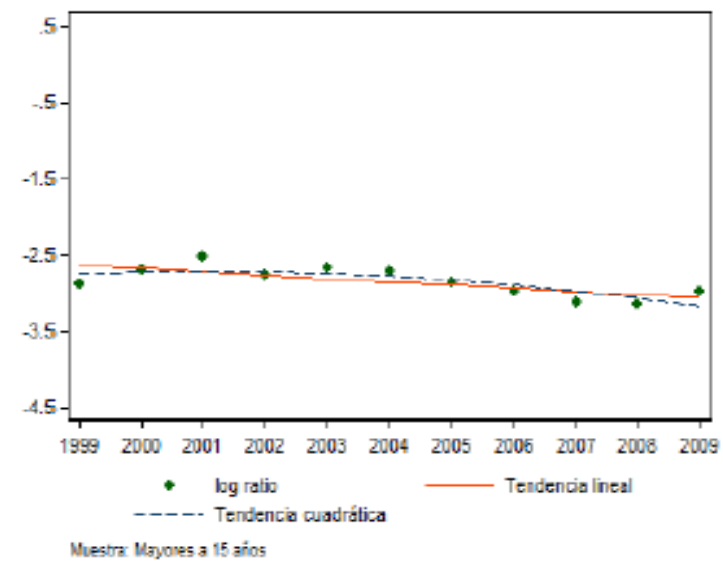

(a) Logaritmo del Ratio de analfabetismo $\left(\frac{\pi}{1-\pi}\right)$

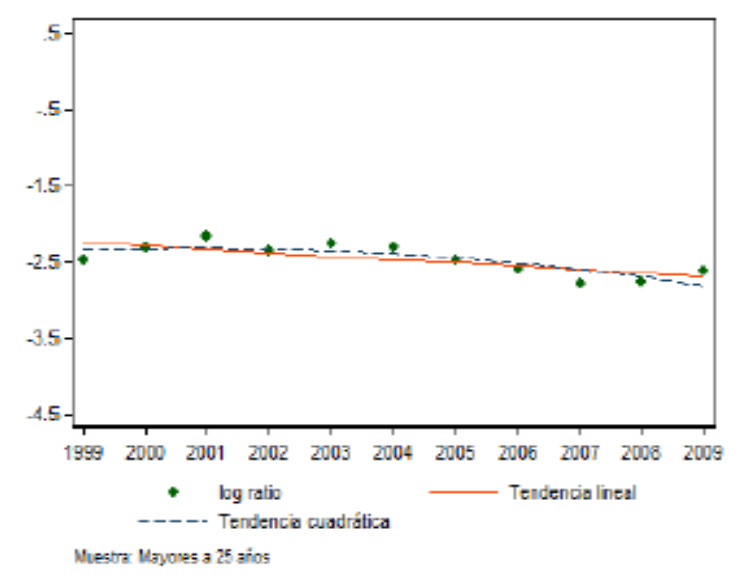

(d) Logaritmo del Ratio de analfabetismo $\left(\frac{\pi}{1-\pi}\right)$

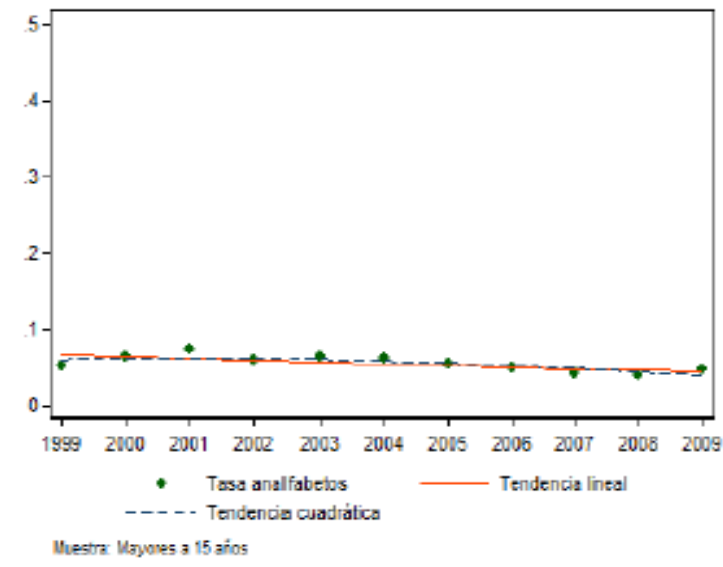

(b) Tasa de analfabetismo $(\pi)$

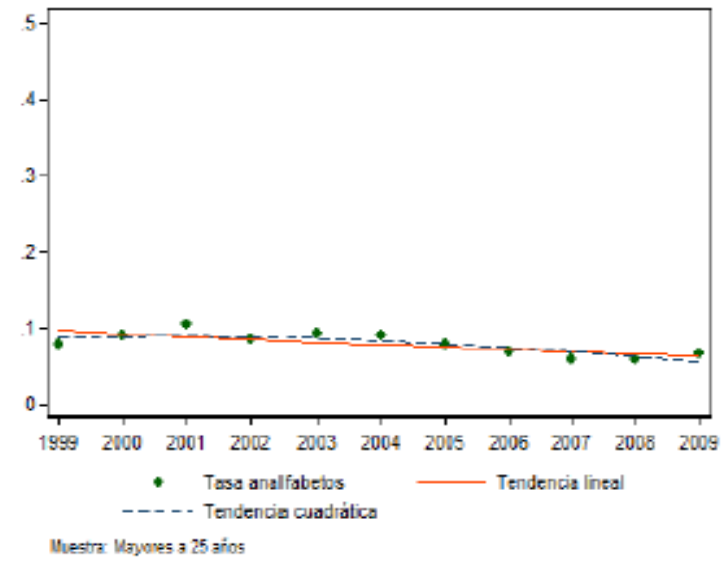

(e) Tasa de analfabetismo $(\pi)$

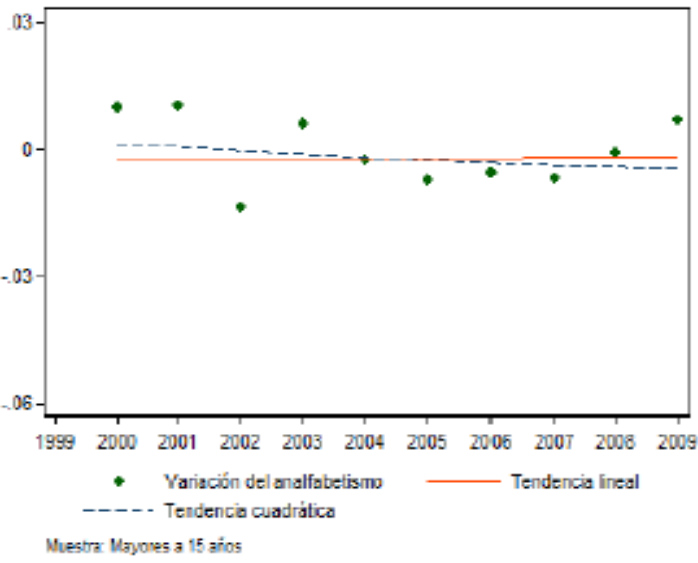

(c) Variación del analfabetismo $\left(\frac{\partial \pi}{\partial t}\right)$

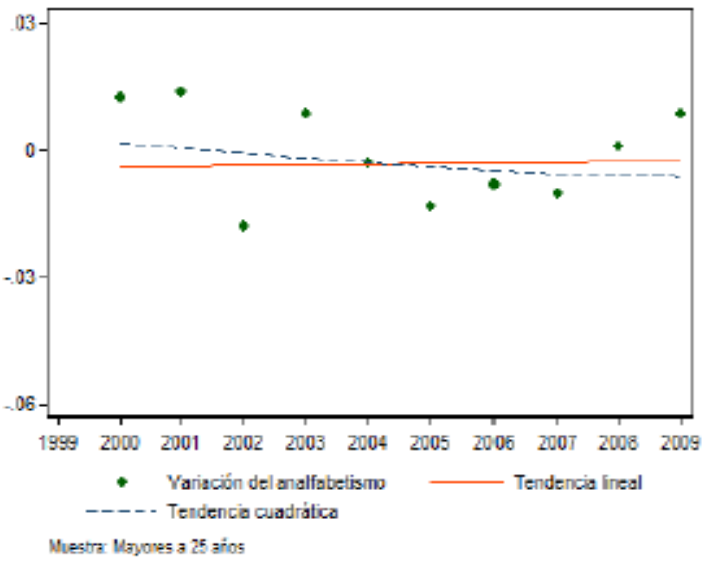

(f) Variación del analfabetismo $\left(\frac{\partial \pi}{\partial t}\right)$

Figura 9: Evaluación de Tendencias. Área Urbana 


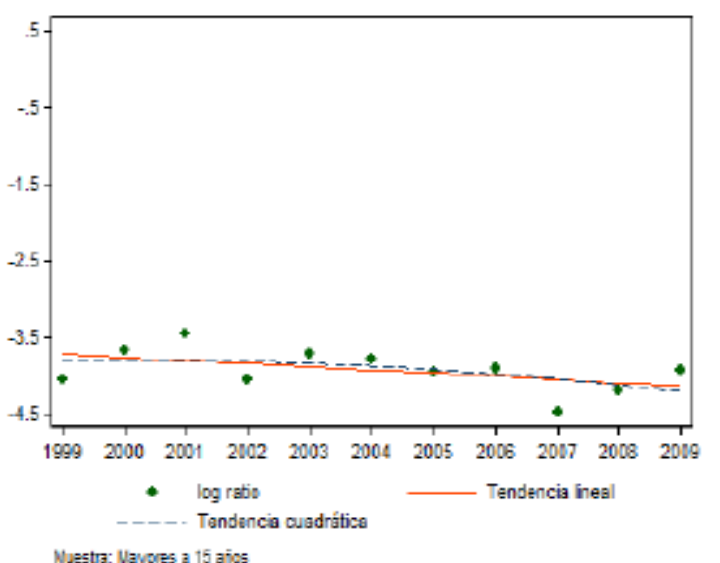

(a) Logaritmo del Ratio de analfabetismo $\left(\frac{\pi}{1-\pi}\right)$

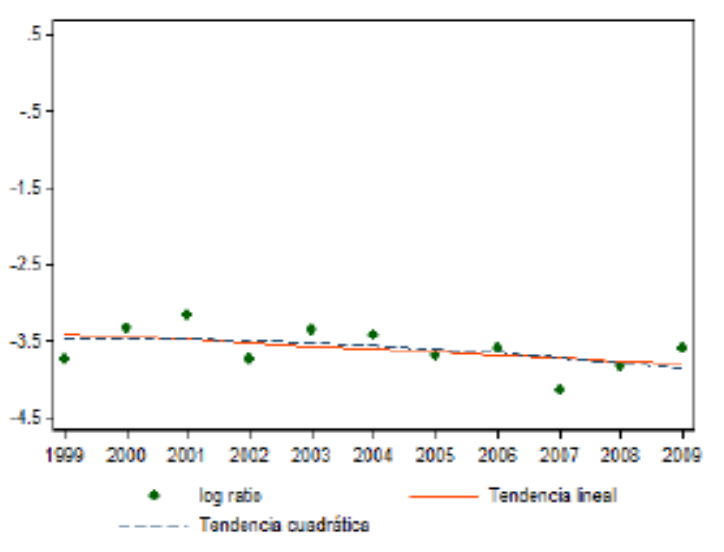

Nuettr: Nayores a 25 aics

(d) Logaritmo del Ratio de analfabetismo $\left(\frac{\pi}{1-\pi}\right)$

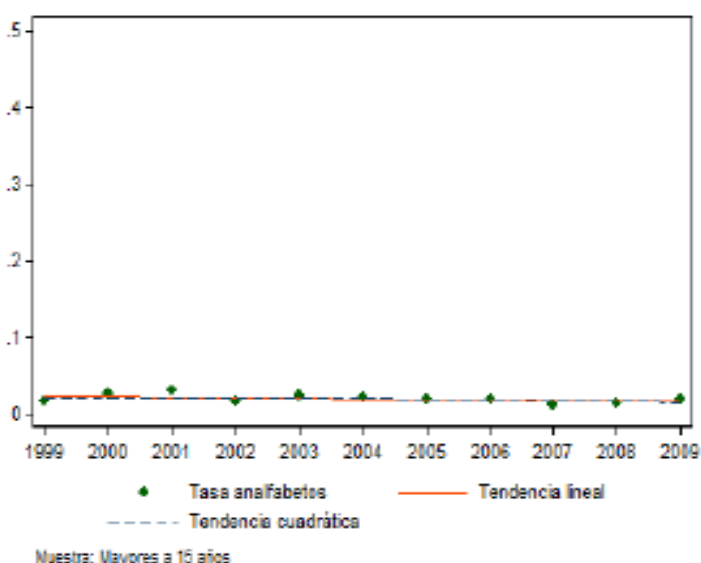

(b) Tasa de analfabetismo $(\pi)$

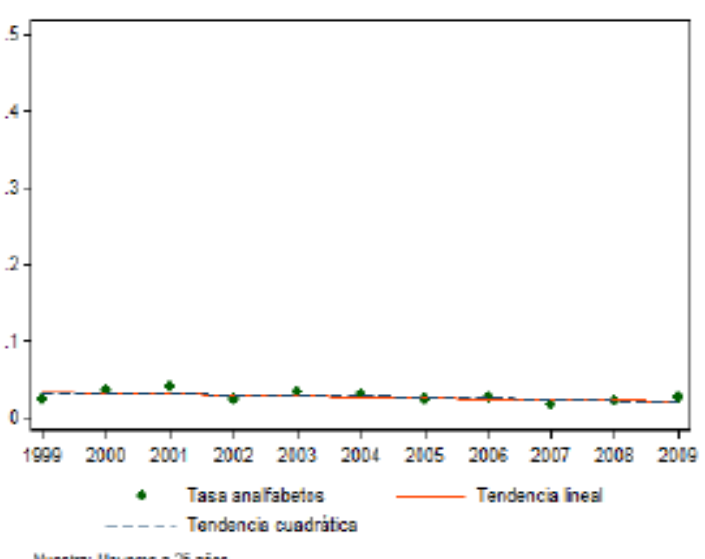

(e) Tasa de analfabetismo $(\pi))$

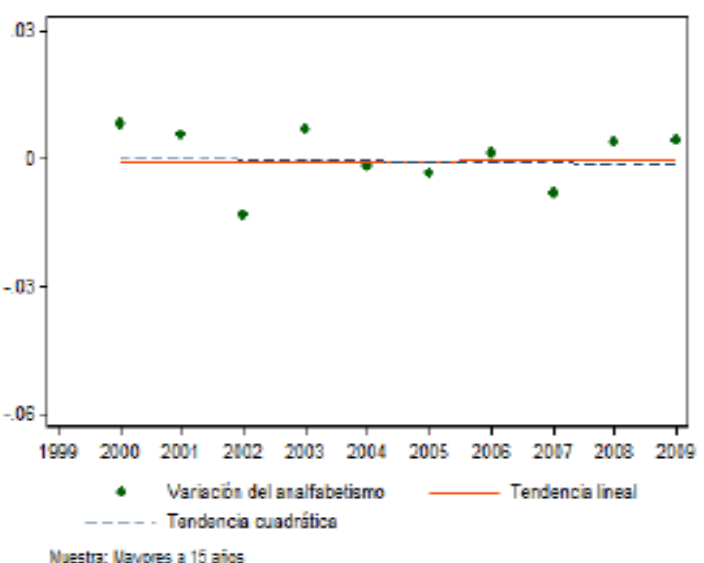

(c) Variación del analfabetismo $\left(\frac{\partial \pi}{\partial t}\right)$

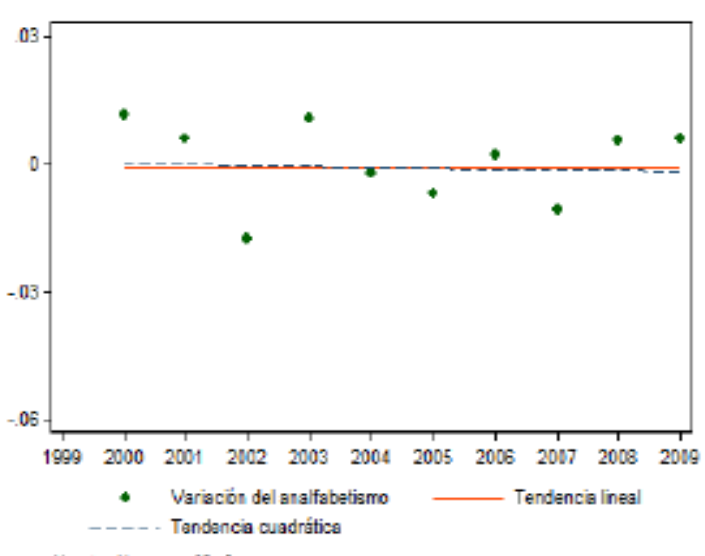

Nuestr: Nayores a 25 anss

(f) Variación del analfabetismo $\left(\frac{\partial \pi}{\partial t}\right)$

Figura 10: Evaluación de Tendencias. Área Rural 


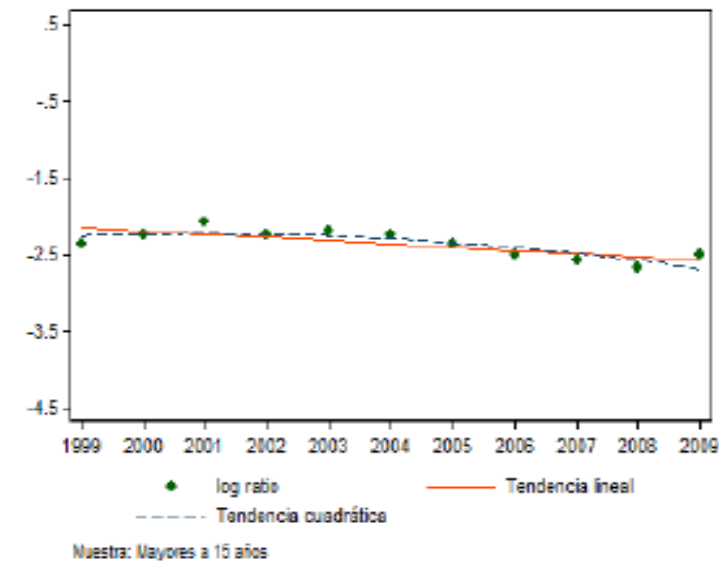

(a) Logaritmo del Ratio de analfabetismo $\left(\frac{\pi}{1-\pi}\right)$

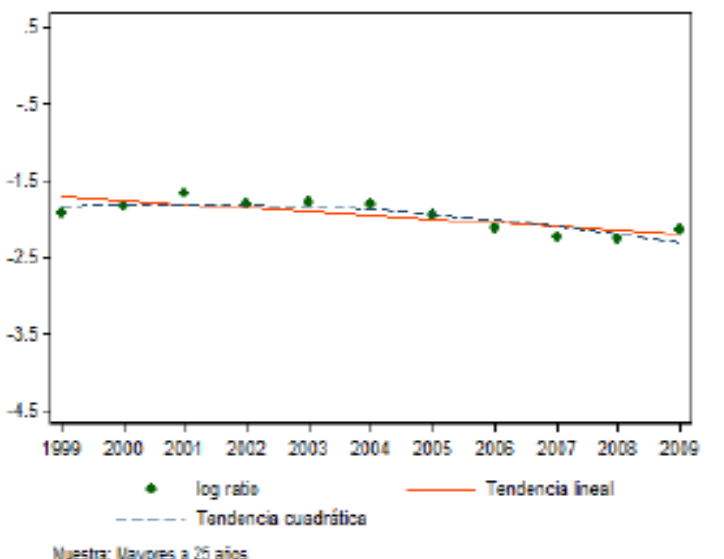

(d) Logaritmo del Ratio de analfabetismo $\left(\frac{\pi}{1-\pi}\right)$

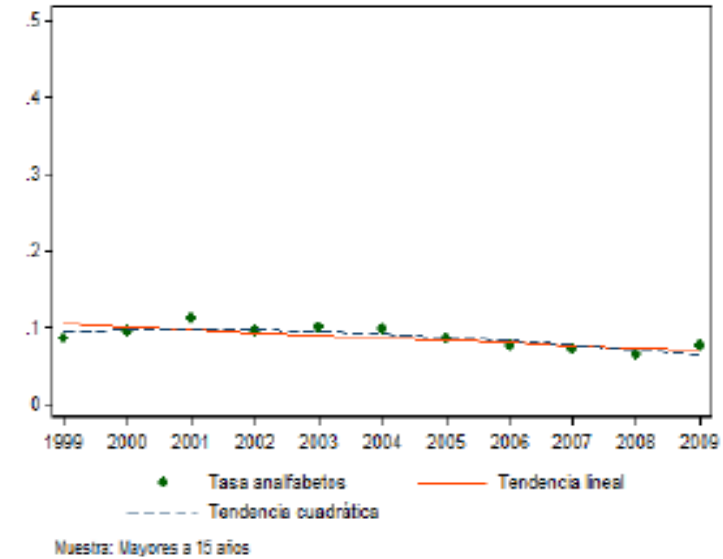

Nuestr: Uayores a 15 ains

(b) Tasa de analfabetismo $(\pi)$

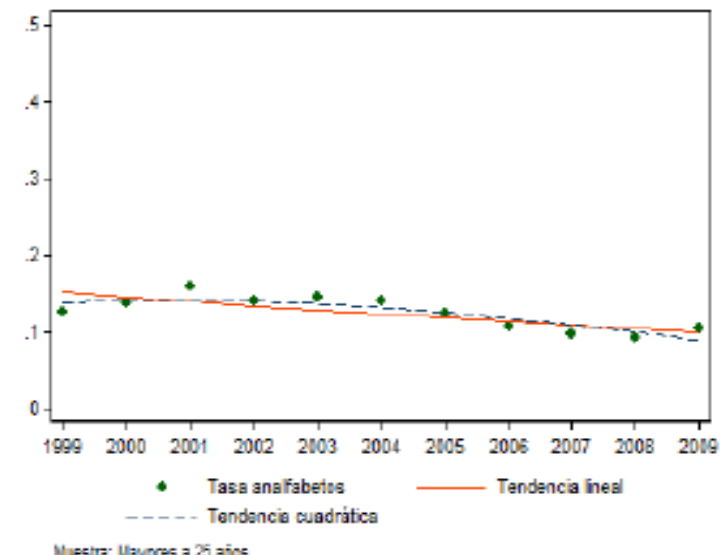

(e) Tasa de analfabetismo ( $\pi$ )

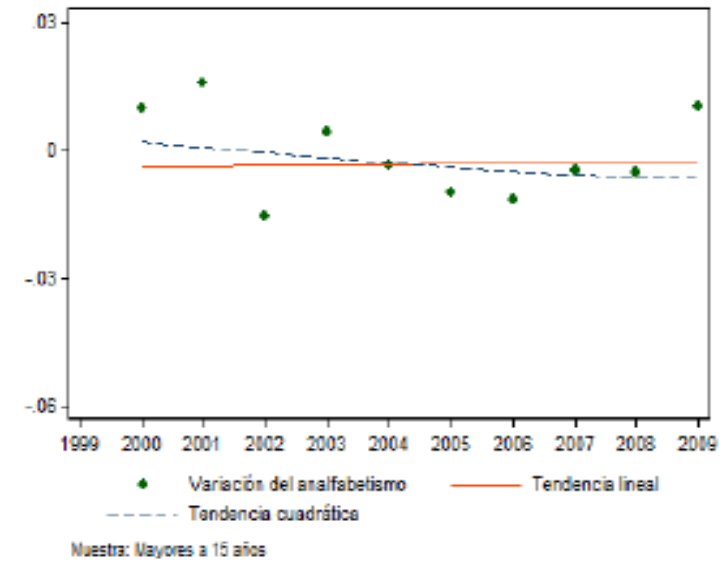

(c) Variación del analfabetismo $\left(\frac{\partial \pi}{\partial t}\right)$

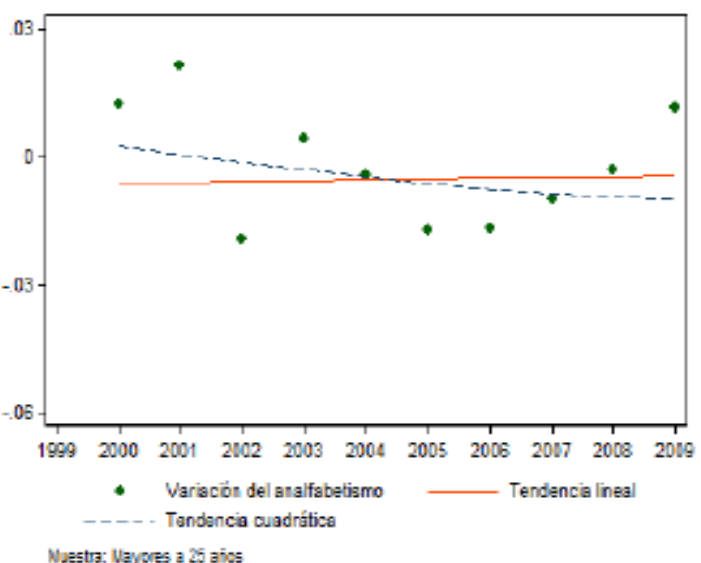

(f) Variación del analfabetismo $\left(\frac{\partial \pi}{\partial t}\right)$

Figura 11: Evaluación de Tendencias. Mujer Urbana 


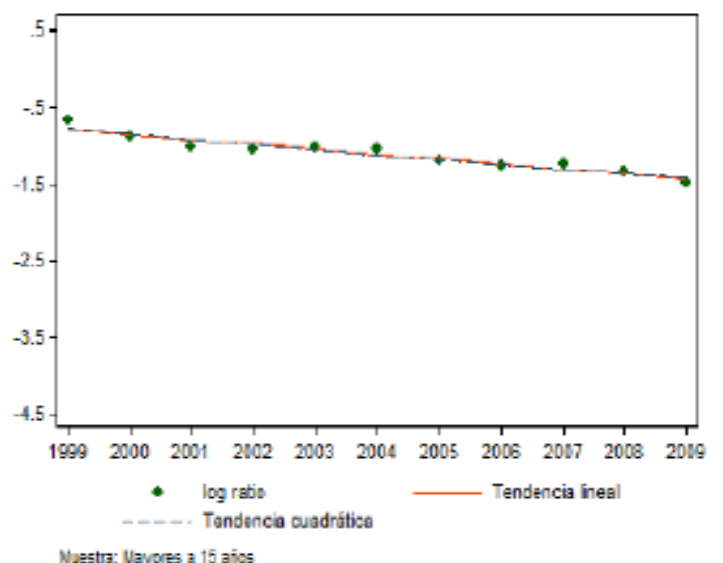

(a) Logaritmo del Ratio de analfabetismo $\left(\frac{\pi}{1-\pi}\right)$

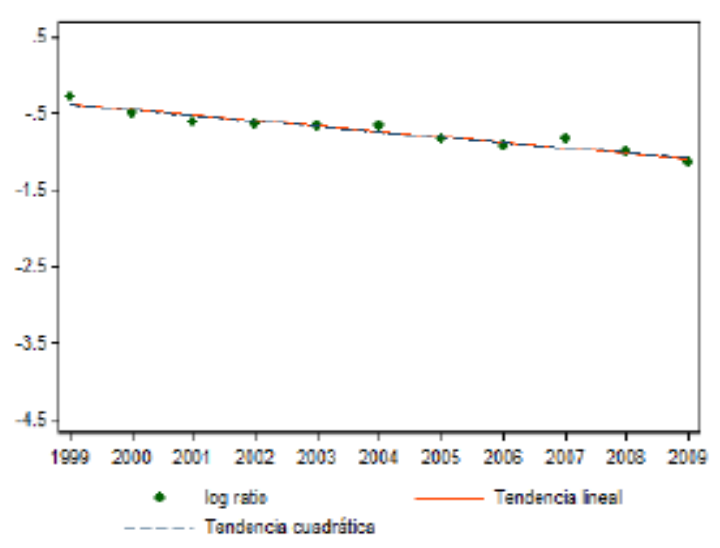

Nuastr: Nayores a 25 anos

(d) Logaritmo del Ratio de analfabetismo $\left(\frac{\pi}{1-\pi}\right)$

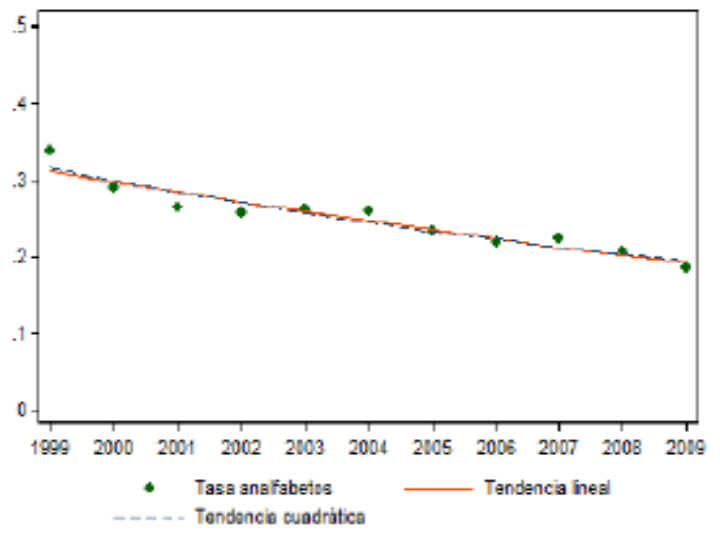

Nuestra: Uayores a 15 ains

(b) Tasa de analfabetismo $(\pi)$

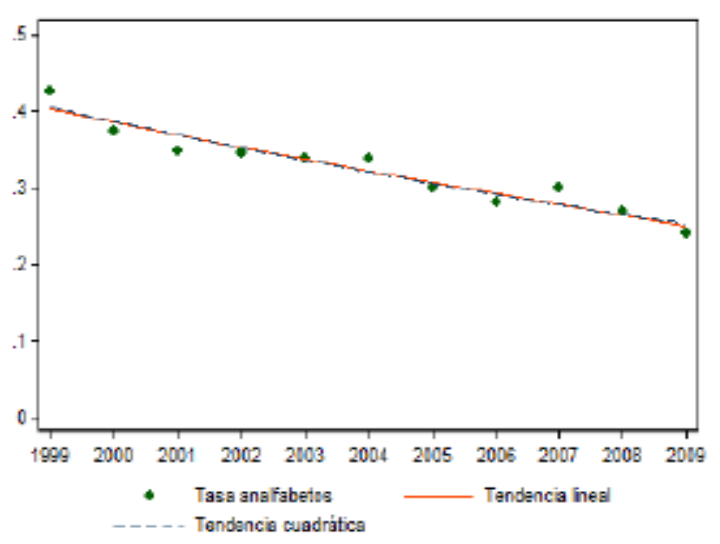

Nuestra: Vayores a 25 ains

(e) Tasa de analfabetismo $(\pi)$

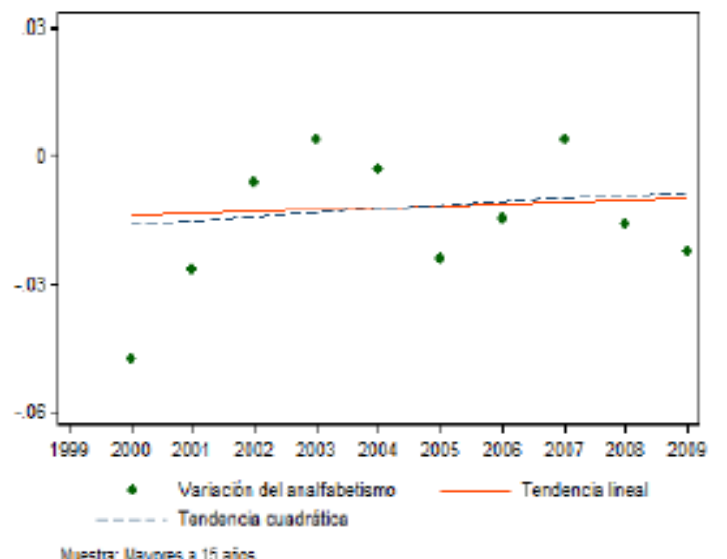

Nuestra: Nayores a 15 ains

(c) Variación del analfabetismo $\left(\frac{\partial \pi}{\partial t}\right)$

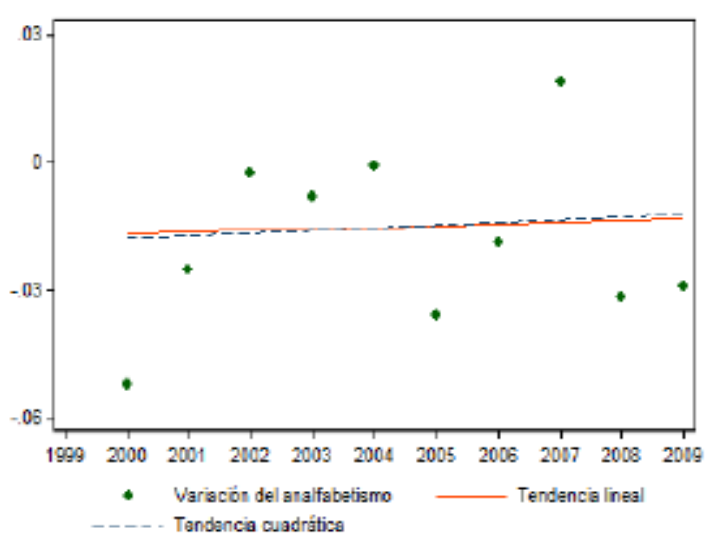

Noestr: Nayors a 25 aiss

(f) Variación del analfabetismo $\left(\frac{\partial \pi}{\partial t}\right)$

Figura 12: Evaluación de Tendencias. Mujer Rural 


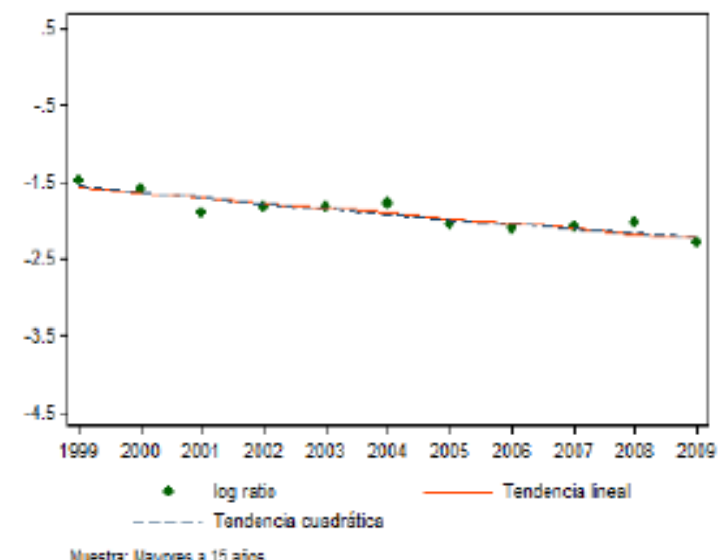

(a) Logaritmo del Ratio de analfabetismo $\left(\frac{\pi}{1-\pi}\right)$

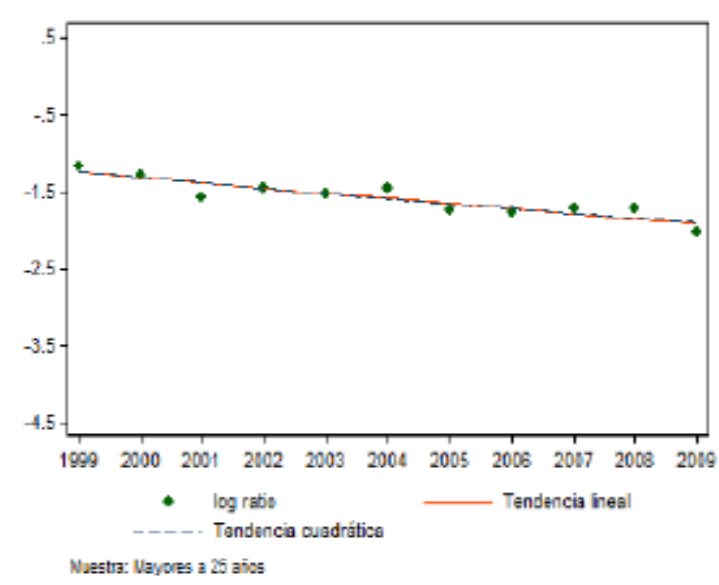

(d) Logaritmo del Ratio de analfabetismo $\left(\frac{\pi}{1-\pi}\right)$

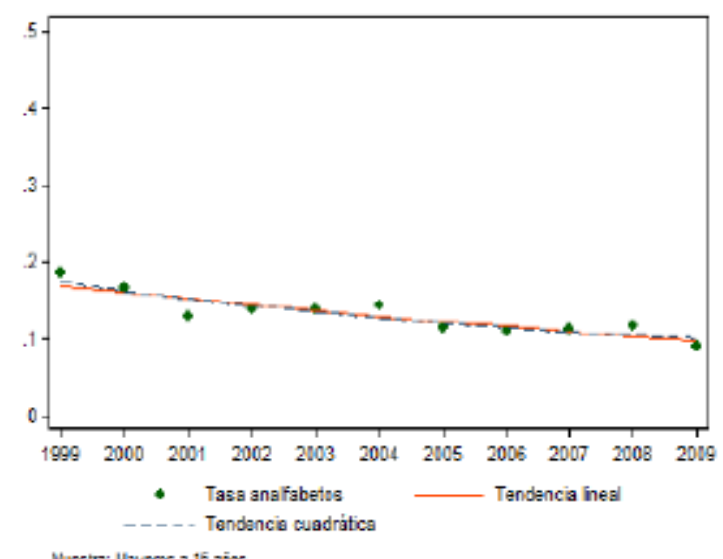

Nuestra: Uayores a 15 ains

(b) Tasa de analfabetismo $(\pi)$

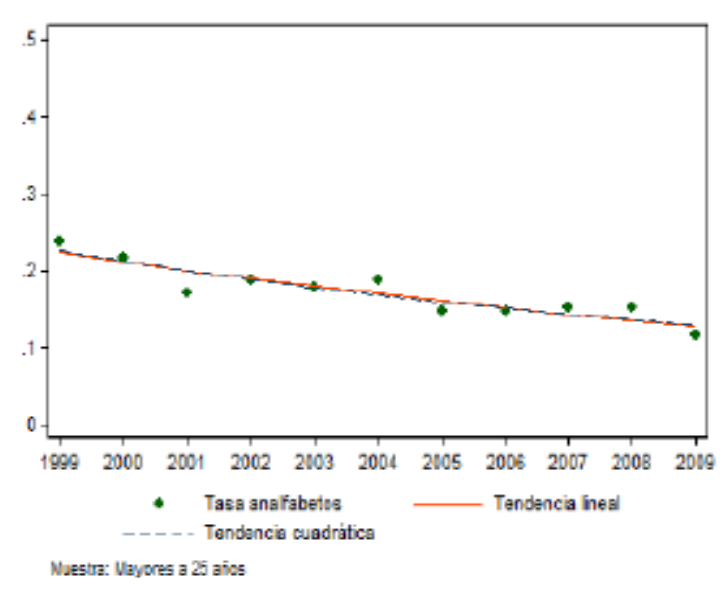

(e) Tasa de analfabetismo $(\pi)$

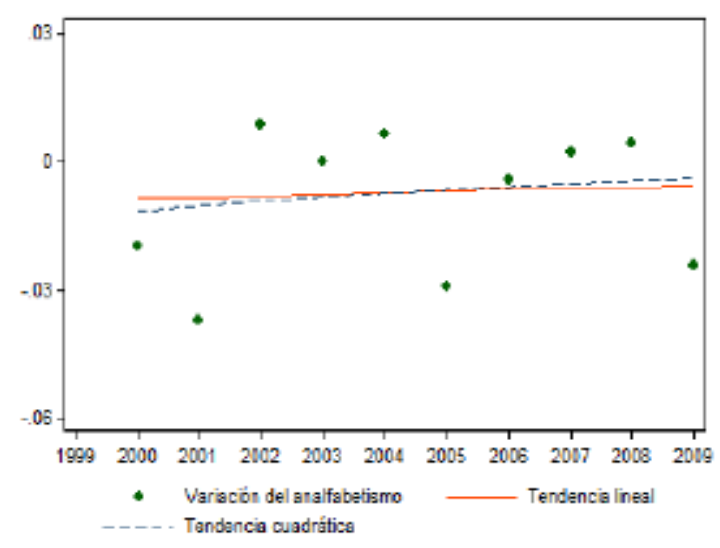

Wastra: Waycess a 15 ans

(c) Variación del analfabetismo $\left(\frac{\partial \pi}{\partial t}\right)$

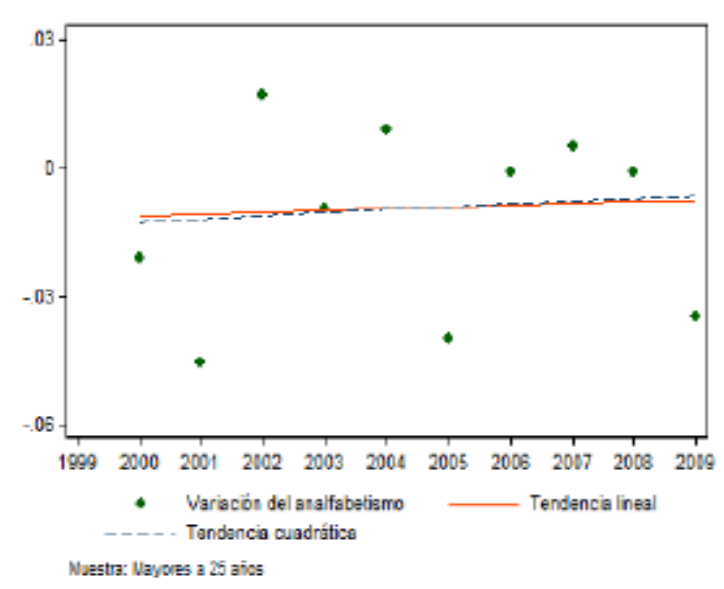

(f) Variación del analfabetismo $\left(\frac{\partial \pi}{\partial t}\right)$

Figura 13: Evaluación de Tendencias. Hombre Urbano 


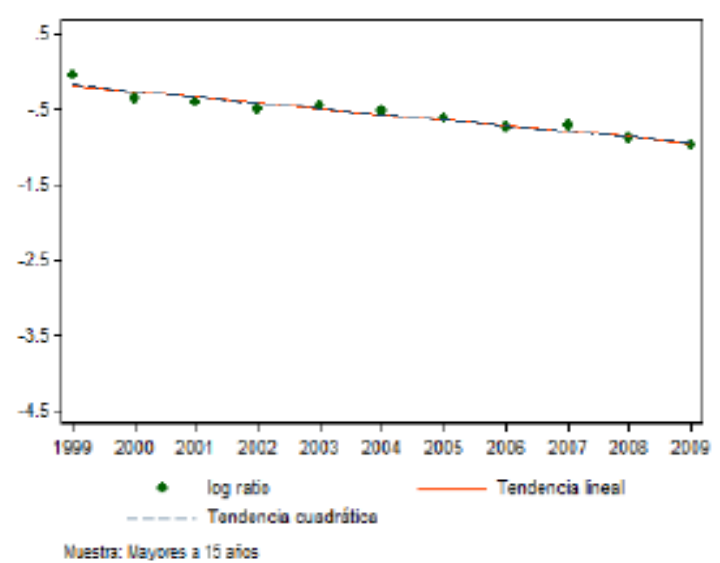

(a) Logaritmo del Ratio de analfabetismo $\left(\frac{\pi}{1-\pi}\right)$

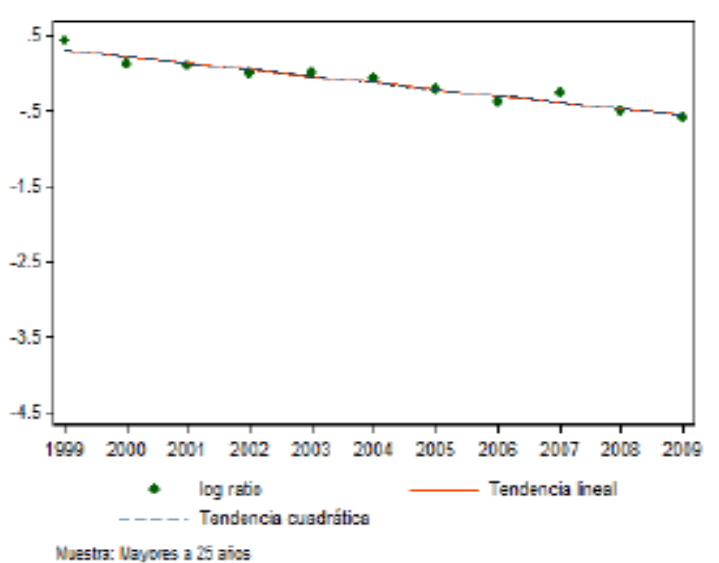

(d) Logaritmo del Ratio de analfabetismo $\left(\frac{\pi}{1-\pi}\right)$

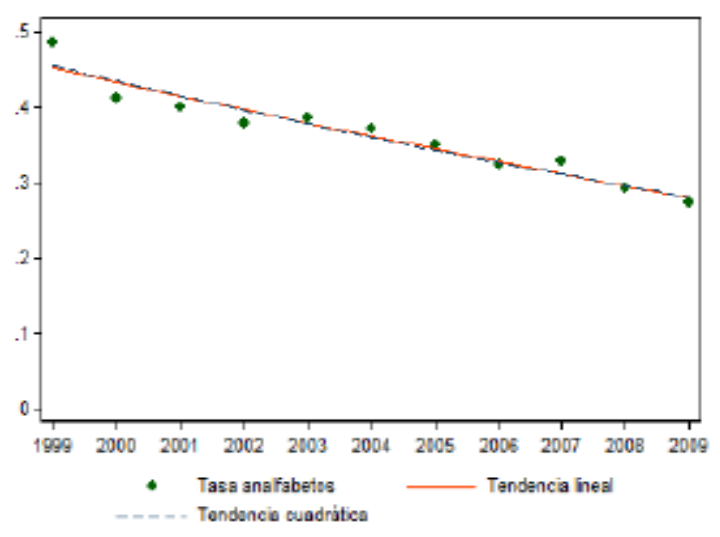

Nuestra: Uayores a 15 aiss

(b) Tasa de analfabetismo $(\pi)$

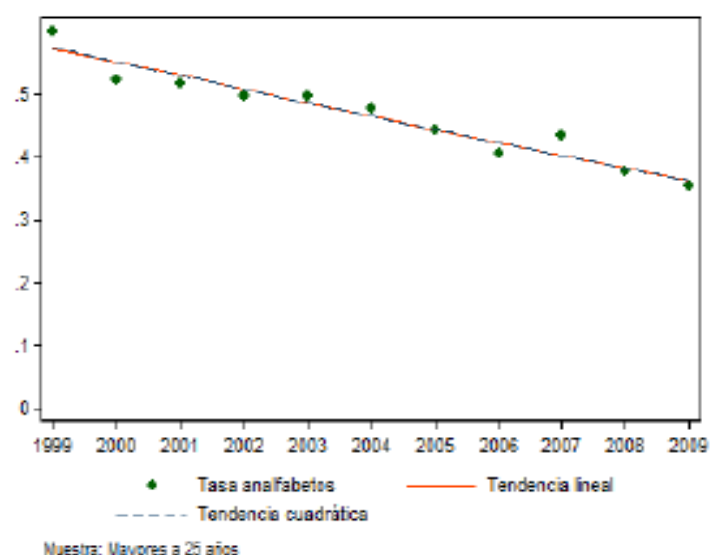

Nuestr: Uayores a 25 aiss

(e) Tasa de analfabetismo $(\pi)$

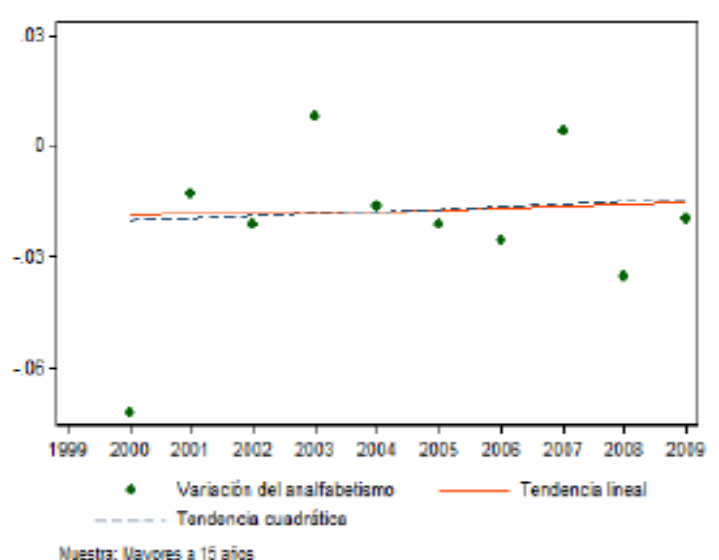

(c) Variación del analfabetismo $\left(\frac{\partial \pi}{\partial t}\right)$

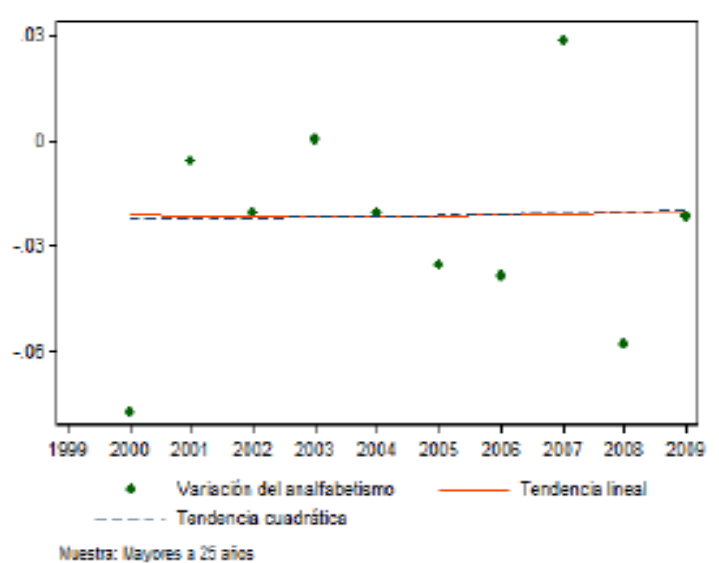

(f) Variación del analfabetismo $\left(\frac{\partial \pi}{\partial t}\right)$

Figura 14: Evaluación de Tendencias. Hombre Rural 


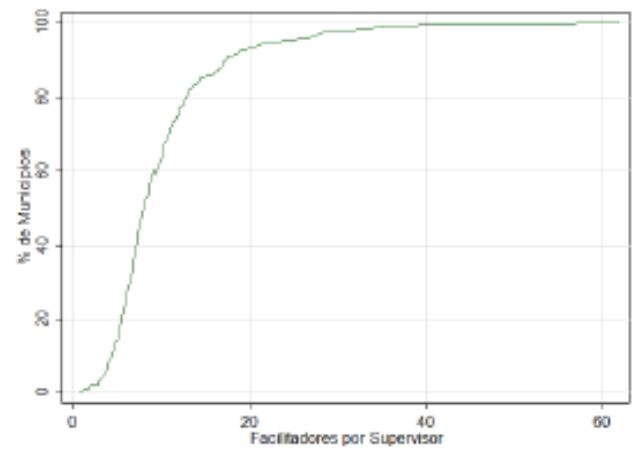

(a) Razón de facilitadores por supervisor

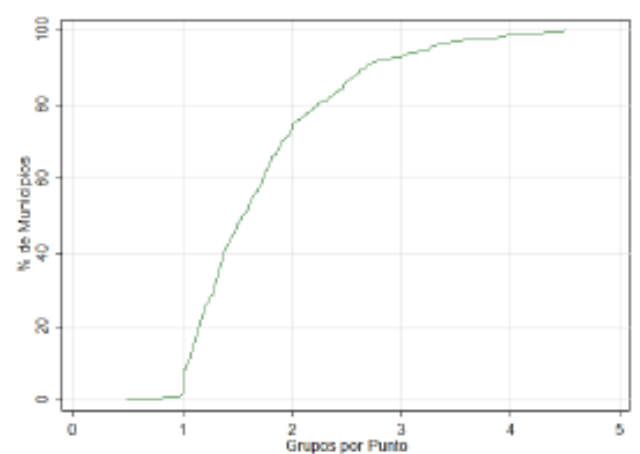

(c) Razón de grupos por punto

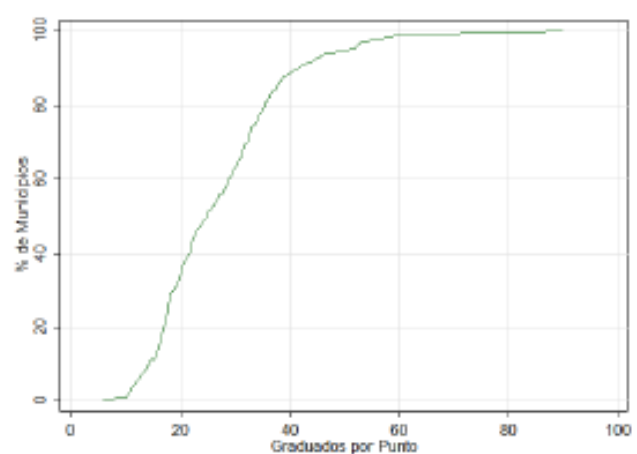

(e) Razón de graduados por punto

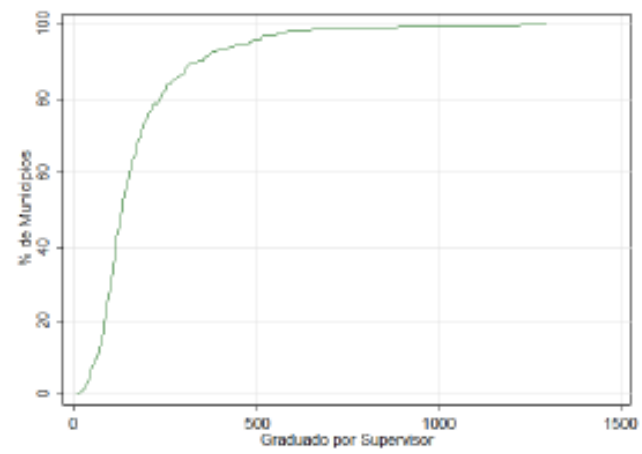

(g) Razón de graduados por supervisor

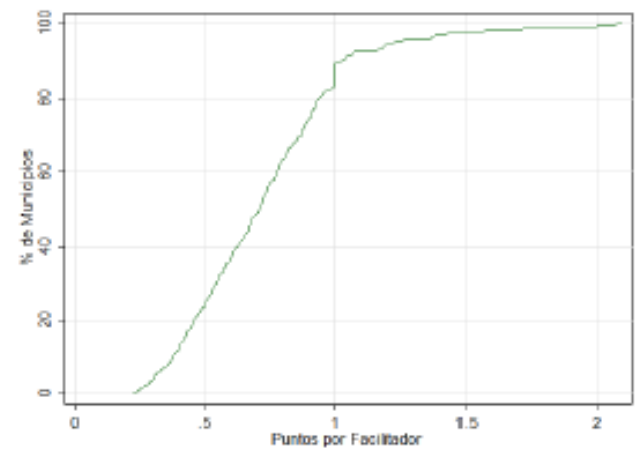

(b) Razón de puntos por facilitador

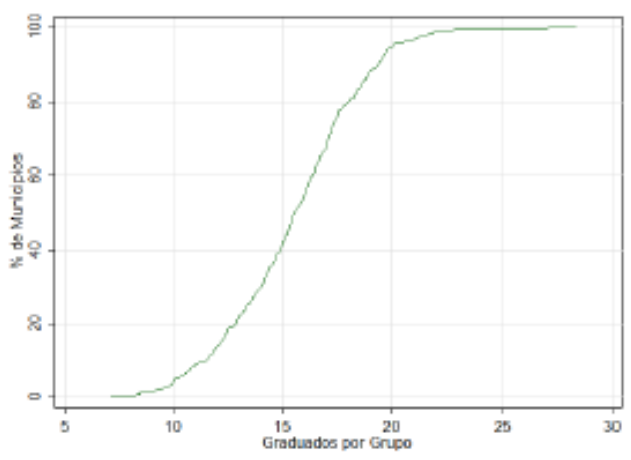

(d) Razón de graduados por grupo

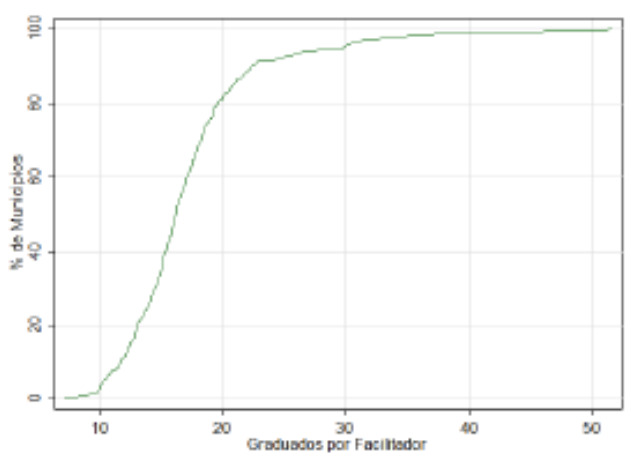

(f) Razón de graduados por facilitador

Figura 15: Distribución de Municipios según Indicador 
Tratados

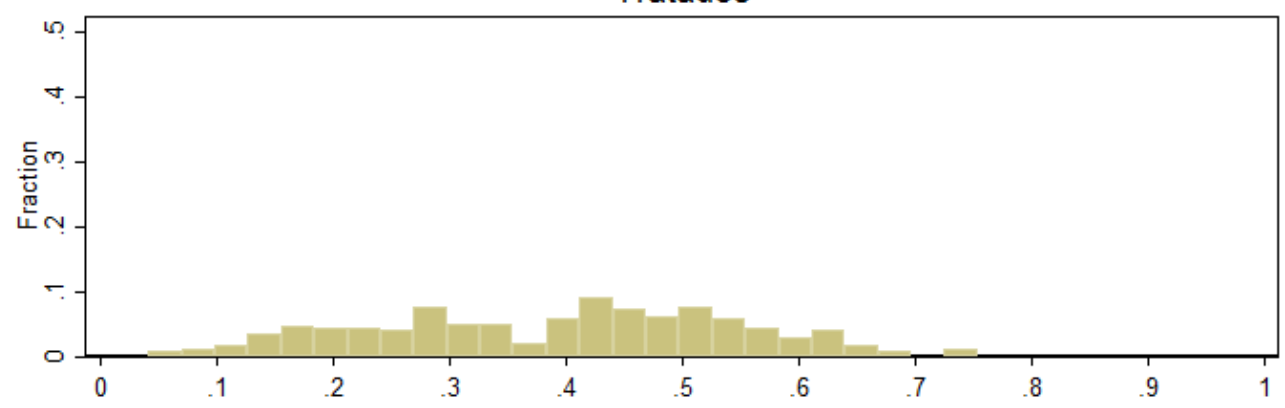

Controles

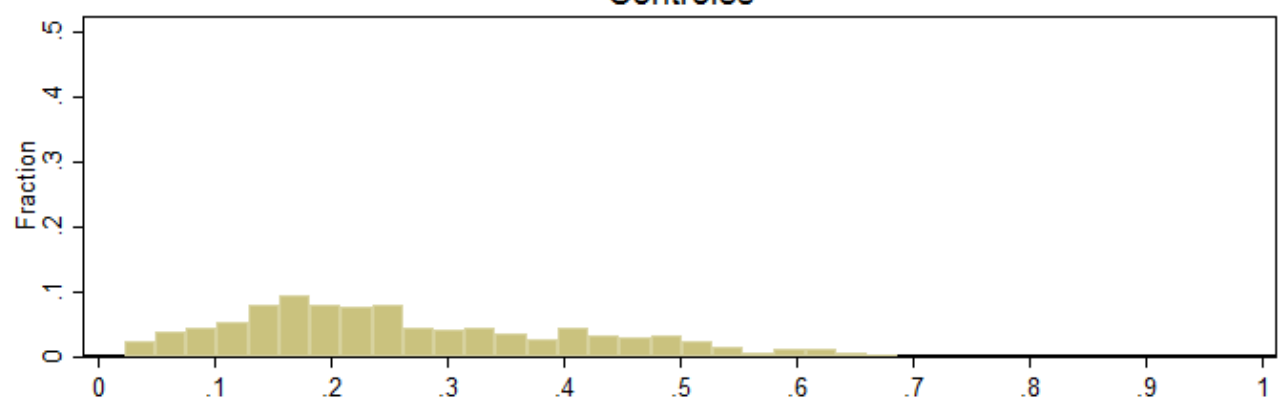

Figura 16: Propensity Scores de la Muestra Completa por Grupo

Tratados

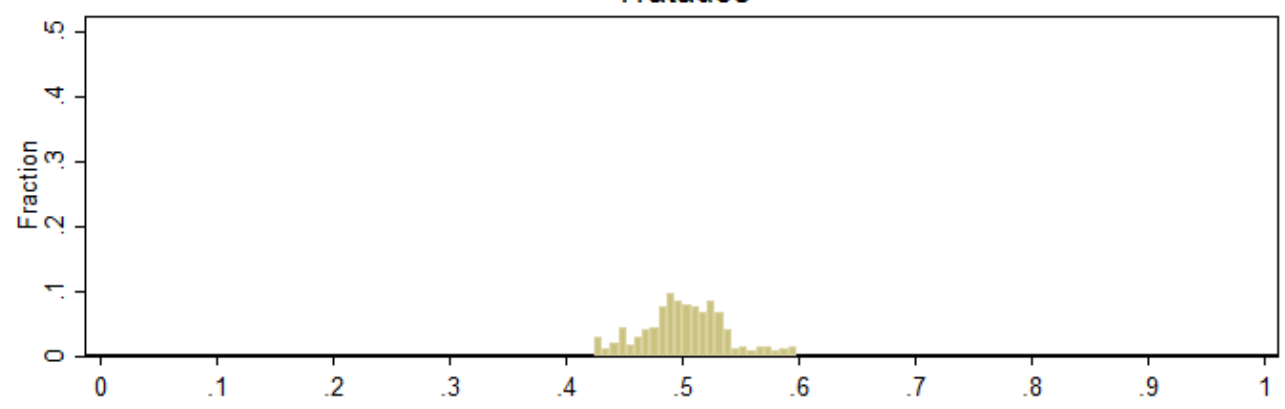

Controles

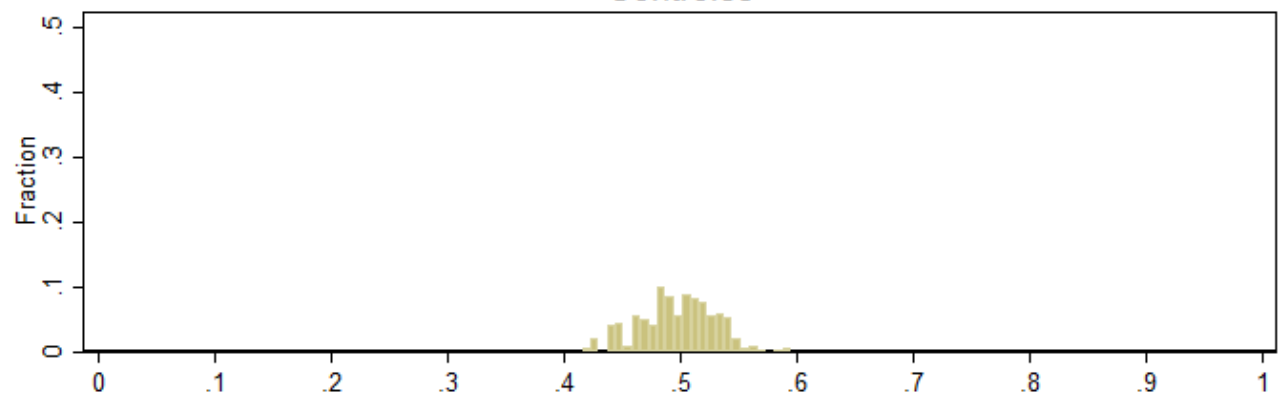

Figura 17: Propensity Scores de la Muestra Emparejada por Grupo 